\title{
Stochastic modeling reveals how motor protein and filament properties affect intermediate filament transport
}

\author{
J. C. Dallon \\ dallon@math.byu.edu \\ Cecil Leduc \\ Institut Pasteur, cleduc@pasteur.fr \\ Sandrine Etienne-Manneville \\ Institut Pasteur, setienne@pasteur.fr \\ Stephanie Portet \\ University of Manitoba, Stephanie.Portet@umanitoba.ca
}

Follow this and additional works at: https://scholarsarchive.byu.edu/facpub

Part of the Mathematics Commons

\section{Original Publication Citation}

Journal of Theoretical Biology

\section{BYU ScholarsArchive Citation}

Dallon, J. C.; Leduc, Cecil; Etienne-Manneville, Sandrine; and Portet, Stephanie, "Stochastic modeling reveals how motor protein and filament properties affect intermediate filament transport" (2019). Faculty Publications. 3689.

https://scholarsarchive.byu.edu/facpub/3689

This Peer-Reviewed Article is brought to you for free and open access by BYU ScholarsArchive. It has been accepted for inclusion in Faculty Publications by an authorized administrator of BYU ScholarsArchive. For more information, please contact ellen_amatangelo@byu.edu. 


\title{
Stochastic modeling reveals how motor protein and filament properties affect intermediate filament transport
}

\author{
J. C. Dallon* \\ Department of Mathematics, Brigham Young University, Provo, Utah, USA \\ Cécile Leduc, Sandrine Etienne-Manneville \\ Institut Pasteur Paris CNRS UMR3691, Cell Polarity, Migration and Cancer Unit, Equipe \\ Labellisée Ligue Contre le Cancer, 25 rue du Dr Roux 75724, Paris Cedex 15, France \\ Stéphanie Portet \\ Department of Mathematics, University of Manitoba, Winnipeg, MB, Canada
}

\begin{abstract}
Intermediate filaments are a key component of the cytoskeleton. Their transport along microtubules plays an essential role in the control of the shape and structural organization of cells. To identify the key parameters responsible for the control of intermediate filament transport, we generated a model of elastic filament transport by microtubule-associated dynein and kinesin. The model is also applicable to the transport of any elastically-coupled cargoes. We investigate the effect of filament properties such as number of motor binding sites, length, and elasticity on motion of filaments. Additionally, we consider the effect of motor properties, i.e. off rates, on filament transport. When one motor has a catch bond off rate it dictates the motion, whereas when motors have the same type of off rate filaments can alternate between retrograde and anterograde motions. The elasticity of filaments optimizes the filament transport and the coordination of motors along the length of the filament.
\end{abstract}

Keywords: Intermediate filaments; dynein; kinesin; vimentin; molecular motors; stochastic

\footnotetext{
${ }^{*}$ Corresponding author

Email address: dallon@math.byu.edu (J. C. Dallon)
} 

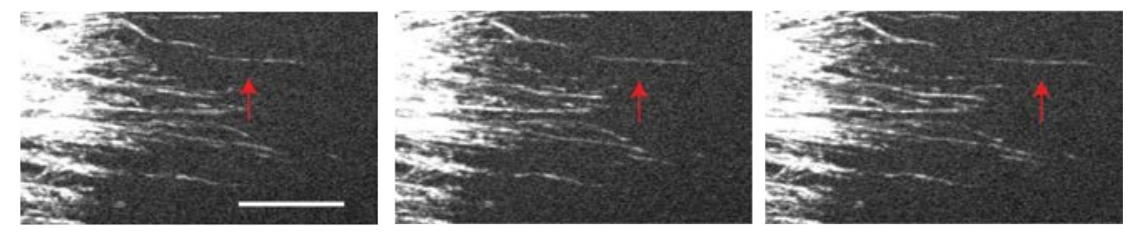

Figure 1: Intermediate filaments motion observed during a FRAP (Fluorescence Recovery After Photobleaching) experiment. The front of an strocytoma cell expressing vimentin-GFP is photobleached over a distance of $20 \mu \mathrm{m}$ from the cell edge. Snapshots show the bleached region 1 minute after photobleaching and were acquired every 10s. The red arrow highlights the motion of a single filament. Bar, $10 \mu \mathrm{m}$.

\section{Introduction}

Intermediate filaments (IFs) are an essential cytoskeletal component of mammalian cells. IF proteins assemble into complex filamentous networks that enable their cell functions. IFs interact with other cytoskeletal components such 5 as, actin filaments and microtubules, via motor proteins or structural linkers [1. Network remodeling dynamics results from the coupling between assembly, disassembly and intracellular transport processes regulated by post-translational modifications of intermediate filament proteins as well as severing and annealing of filaments. Intracellular transport of IFs has been identified as a key process for the network dynamics; see, e.g., 2, 3, 4, 5, 6, IFs regulated interactions with motors and structural linkers result in different modes of motility for assembled IF proteins in cells: slow, fast, bidirectional, retrograde, anterograde, and/or switching motions [7. For instance, vimentin motility in astrocytes is controlled by a combination of actin dependent flow and microtubule-driven transports [2]

15 (see figure 1). Furthermore, when astrocytes initiate migration, the intermediate filament dynamics switch from a balanced combination of retrograde and anterograde transport to a mostly anterograde transport of filaments during cell polarization [2. Contributions and regulations of motor protein-driven transport, actin dependent retrograde flow, and the association with structural linkers $20 \quad$ are still not well understood.

There is a rich theory and history modeling motor protein-driven transport. Ever since the discovery of the motor protein kinesin-1 [8, 9] and the role of dynein as a motor molecule responsible for intracellular transport [10, 11], they have been widely studied and modeled. Typically the research relates to

25 properties of the molecules, vesicle transport, and the cooperative behavior of motor proteins [12]. A model for vesicular tranport was introduced in [13] where opposing motor proteins are allowed to stochastically bind and unbind to the microtubule so the number of attached motors fluctuates. A tug of war ensues where both motors can back step, have load dependent off rates and velocities, 30 and motors of the same species equally share the load. In [14, they call this the bidirectional mean field model and simulate experiments using catch bond off rates for both motor types. In more recent work [15] the authors investigate the affect of an asymmetrical detach rate for dynein using a stochastic model 
which includes a spring like interaction for the dynein linking to a single cargo.

35 Comparatively little has been done in relation to how these motor molecules interact and transport IFs or elastically coupled cargo.

Here we propose a model which serves both purposes: models transport of IFs by antagonistic motor proteins and explores how elastic coupling of cargo affects motor proteins dynamics and transport. Our focus is on the motor 40 protein-driven transport of long filaments along microtubules. Developments of techniques for imaging living cells now allow the tracking of single filaments in cells [4]; however, still a lot of questions are not yet experimentally tractable such as the number of binding sites of motors along the IFs and whether length and elasticity affect filament transport. We propose a mathematical model for 45 the motion of a single filament driven by antagonistic motor proteins to answer these questions as well as how does a cell regulate the motion of IFs. In the literature there is experimental evidence for both catch bond and exponential off rates for kinesin and catch bond off rates for dynein [16, 14. Yet modelling dynein with an exponential off rate provides good theoretical description for

50 some experiments [17. Thus we also investigate the effects of the combinations of these two off rates on the system.

Mathematical models based on population dynamics approaches have been proposed to decipher the collective motion of IFs in cells; see e.g. [18, 19, 20, 21, 22. However, to the best of our knowledge, there is only one model for the

55 motion of IFs associated with microtubules and it is concerned with segregation of axonal microtubules and neurofilaments 20. There the authors considered axonal movement of neurofilaments and organelles within a cross-section of the axon. Thus the neurofilaments are modeled as discs which change size due to lateral motion.

60 Here, we develop a mean-field load sharing model of intermediate filament transport by modifying the stochastic model for vesicle transport given in [13, 14. Taking into account the remarkable elastic properties of IFs, they are modeled as nodes coupled by springs. The motor molecules can interact with the nodes. Thus instead of considering a single cargo we consider multiple 65 cargoes which are force coupled by springs and we consider the effects of a drag force on the filament. Although the application here is IFs, the model is more widely applicable to any system of coupled transport by motor proteins.

The simulations are designed to reveal the effects of filament and motor properties on IF transport. The main motor property investigated is the off 70 rate. In cells, the repartition of active motor proteins, dynein and kinesin, is not well known and not necessarily homogeneous/uniform; at some intracellular locations only a few active motors could be available whereas at other locations, active motors could be abundant [23, 24, 25]. IFs exhibit polymorphism in length and diameter [26. Bundling results in fibers of different diameters and 75 various elasticities [27. We investigate the effect of the elasticity of the filament (from the broader perspective of transport by motor proteins, the strength of the coupling between cargoes), the initial length of the filament (the number of coupled cargoes), the maximum number of binding sites for motors, and different profiles for the detachment kinetics of the motor molecules including 


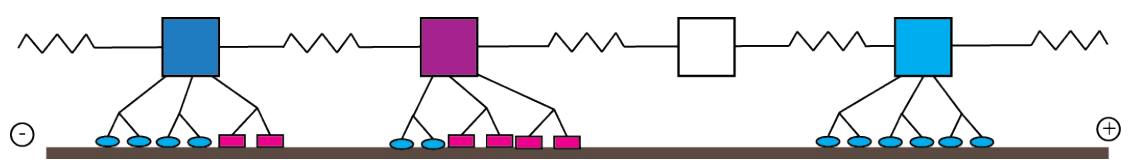

Figure 2: A caricature of the model. The intermediate filament is a series of nodes connected by springs. The microtubule is represented by the thick line. The filament interacts with the microtubule via motor molecules such as dynein and kinesin which can attach and detach to the nodes. Kinesin is represented as magenta and dynein as cyan. The node color is determined by a weighted average of the number of each type of motor molecules and will be used in future figures.

the known catch-bond feature of dynein's detachment kinetics [14, 28, 29, 16, 30]. The modeling reveals that intermediate values of elasticity and more binding sites for motor molecules facilitate motor coordination and persistant directional transport along the length of the filament.

\section{The Model}

The model is a one-dimensional stochastic model which considers the motion of an intermediate filament along a microtubule due to the tug-of-war between dynein-1 and kinesin-1. The filament is modeled as nodes attached by springs (see figure 2). Both dynein and kinesin can bind to the same node at the same time, thus causing the tug-of-war scenario on each node. The motion of the filament is determined by the motion of each node and their coupling. The motors attach and detach in a random manner allowing the number of each type of motor attached to a node to change with time. There are $N$ nodes on each filament. The equations of motion are determined by setting the sum of the forces for each node equal to zero (see Section 57. Each node has forces 95 acting on it due to drag, motor molecules and the springs which link it to other nodes on the filament. The forces due to the motor molecules are described similarly to the model for a single cargo developed in [13] where they assume the number of forward moving motors (kinesin) bound to a cargo is $n_{K}$ which stochastically changes. In a similar manner $n_{D}$ is the number of backward moving motors (dynein) attached and both motor types can step backward. In our model, as in theirs, motors of the same type equally share the load. We have two modifications to how they modeled the system which are necessary since we are modeling a filament. The first difference is we allow forces in the direction of motion (the attached springs may pull in the direction of the motion of the motor molecules) and the second difference is we consider the drag force on the node. The forces in the direction of the motion do not assist the motor molecules (see methods section and figure therein).

To summarize, each filament is composed of $N$ nodes which are connected by springs. On each node $n_{k}$ and $n_{d}$ denote the variables which change with 110 time and represent the number of attached motors of kinesin and dynein which are interacting with both the node and the microtubule. The variables $n_{k}$ and $n_{d}$ should not be confused with the parameters $N_{K}$ and $N_{D}$, which are 
the maximum number of dynein and kinesin which can be attached to each node and are constant for a particular simulation. When we refer to attached motors we mean motors which are interacting with both the filament and the microtubule. The filament starts off with a fixed length which changes due to the elastic properties of the filament and the forces acting on the filament. Unless otherwise stated, references to filament length mean the initial length of the filament. For more details see the methods section.

120 Important parameters which are varied in the simulations fall into two categories: filament properties and motor properties.

Filament property parameters:

- $N$ - filament length. $N$ is the number of nodes on a filament and determines the length of the filament when there are no forces. The nodes are separated by $0.5 \mu \mathrm{m}$. The value $N$ is varied from $2,5,10,15,20,25$, and 30 nodes. This corresponds to filament lengths ranging from 1 to $15 \mu \mathrm{m}$ in the absence of any forces. We assume each node, including both end nodes, represents a length of filament.

- $\alpha$ - filament elasticity. The normal filament elasticity is $\alpha=0.345 \times$ $10^{-8} \mathrm{~N} / \mu \mathrm{m}$ which corresponds to $22 \mathrm{MPa}$. This lies in the range of experimentally measured values $31,32,33$ and compares well with values used in [34. In the simulations we use three values for fiber elasticity - less stiff filaments with $0.001 \alpha$, normal filaments with $\alpha$, and stiff filaments with $1000 \alpha$.

- $N_{D}, N_{K}$ - the maximum number of motors allowed per node. The maximum number of motor molecules of both types allowed, $N_{D}=N_{K}$ is varied from 2, 4, 16, and 32 for each node and represent the maximum number of binding sites on a region of a filament (represented by a node) allowed for each type of motor.

Motor property parameter:

- Off rates. For each motor type, the off rate can be either exponential or catch bond. Exponential off rates have been used to model both kinesin and dynein. There is experimental evidence to suggest that both dynein and kinesin exhibit catch bond behavior [14, 30]. The functional forms per motor are shown in figure 2 and the functions are given in equations 15 18

Finally we consider the initial conditions, which specify the cellular context (the number of motors of each type initially attached). Four initial conditions 150 are used: 1) no motor molecules initially attached, 2) filaments with $N_{D}$ dynein initially attached and no kinesin, 3) filaments with no dynein and $N_{K}$ kinesin initially attached, and 4) filaments with $N_{D}$ dynein and $N_{K}$ kinesin initially attached. 
Kinesin exponential

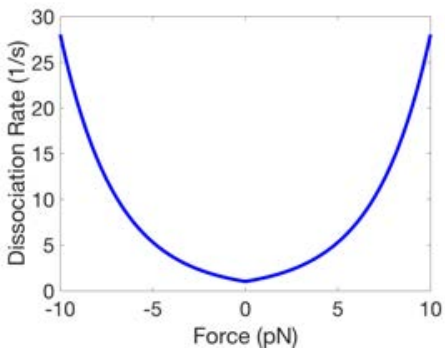

Dynein exponential

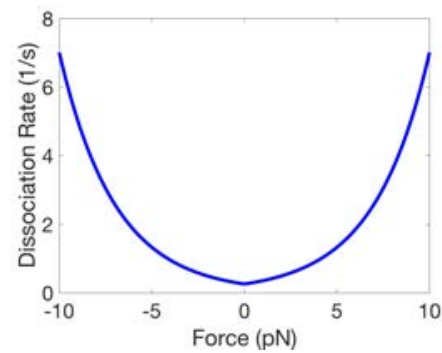

Kinesin catch bond

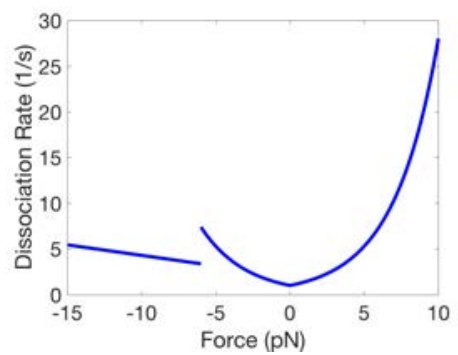

Dynein catch bond

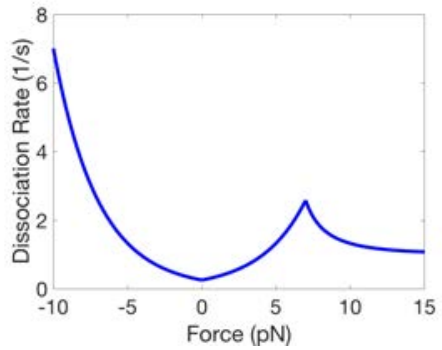

Figure 3: Graphs of the off rate functions.

\section{Results} any forces), elasticity, maximum number of motor molecules, detach rates, and initial conditions affect the system. We divide the results into two parts: how the motor properties affect the system and how the filament/cargo properties affect the system.

160

To analyze the simulations we investigate the filaments mean speed, instantaneous velocity at the end of the simulation, the number of dynein and kinesin motors attached to each node, the average number of dynein and kinesin attached to each filament, and the position of the first filament node.

\subsection{How the data is presented}

165

The mean speed of a filament is the total distance traveled by the filament including backtracking divided by the time. Thus it is an averaged speed for each filament based on the total distance traveled. Let $x_{1}(t)$ be the location of the first node at time $t$. The speed is $\sum_{i=2}^{n}\left|x_{1}(i \Delta t)-x_{1}((i-1) \Delta t)\right| /(n \Delta t)$ where $n$ is the number of time steps and $\Delta t$ is the time step. The average or expected speed is the speed averaged over all realizations.

The velocity is an approximate instantaneous velocity at the end of the simulation averaged over all simulations. It is calculated by taking the location of the first node in a filament at the end of the simulation minus the position of the first node in the filament at the previous time step divided by the time 

filament.

The number of dynein or kinesin at the end of the simulation (normalized so the maximum is 1 ) is determined by finding the best line fitted to the averaged number, over all nodes and all realizations, for the last 400 seconds of the 180 simulation using linear least squares, see figure 4 panel (B) and (E). That line is evaluated at the final time of the simulations to determine the value.

Unless otherwise stated all the simulations results are shown after 100 seconds to allow the system to move away from the initial conditions. Three hundred realizations are simulated for each parameter set. Each simulation is run for 1600 seconds.

The results are analyzed by showing the number of motor molecules on the filament (normalized by the maximum number allowed) at the end of the simulations and an indication as to whether this is a "steady state value" or a transient value (Figures 5, 7, and 11). Additionally, the average speed of a filament (Figure 10) and the position of the first node of a filament (Figure 8 are plotted.

In panels (A), (B) and (E) of figure 4 , the normalized number of dynein and kinesin, $n_{D} / N_{D}$ and $n_{K} / N_{K}$, is averaged over the realizations for each node of a filament; the number of dynein and kinesin is given as function of time.

195 Thus the plots have two curves for each node. One curve is for the dynein (cyan curve) and one is for the kinesin (magenta curve). In most figures the curves are superimposed and indistinguishable.

In panel (D), the normalized number of dynein and kinesin as a function of time for 16 realizations and the average over all realizations (as in panel (A) and $(\mathrm{B})$ ) are visualized in the following way. Each filament is depicted as a vertical color bar extending in the horizontal direction (which is time). The 16 realizations are plotted in the vertical direction with the top color bar being twice as wide as the other and depicting the average of all realizations. In these images the color depicts the number of kinesin and dynein for each node with 205 the first node in the filament plotted at the bottom of each strip and the last node plotted at the top of each strip. The width of the color bars from plot to plot is normalized so that each filament is the same width regardless of the filament length (or number of nodes). Cyan represents only dynein attached, magenta represents only kinesin attached, white represents no motors attached, and blue represents equal proportions of both motors.

In panel (B) and (E), the data for the tail end of the dynein (cyan) and kinesin (magenta) curves is fitted by least squares to find the best fit line which determines the final amount of motor molecules and whether the number is near a steady state. Using this information, the final average proportion of motor molecules is estimated and plotted as a box. Its interior color, that follows the same color scheme as in panel (D), indicates the proportion (figure 4 panel (C) and $(\mathrm{F}))$. The outline color of the box indicates whether dynein or kinesin is greater. If the outline is thicker it indicates that the simulation is at or close to a steady state. If the change in the value of either motor molecule over the 
percent of the end value, the simulation is deemed to be at steady state and the border of the square is thicker.

\subsection{Motor properties affect filament transport}

We start with simulations designed to test the effect of the motor properties.

\subsubsection{Motors with catch bond behavior dominate}

The effects of different off rates can be seen in figure 5. When one motor is exponential and one is catch bond, the motor molecule associated with the catch bond off rate determines the direction of the filament as can be see in panels (b) and (c). When both off rates are catch bond, the filament ends up with a significant number of both motor types as indicated by the purple in panels (a). The magenta borders in panel 1(a) indicate that there are more kinesin motors than dynein. When the maximum number of allowed motors is low, $N_{K}=N_{D}=2$, the stochastic effects play a role (see also the last rows in all panels of figures 22 and 27 in the supplemental data).

When both off rates are catch bond, figure 6 shows the velocity, speed, and position of the filaments. In panel 1 (c) all the fibers are stuck. In panels 1 and 2 the filaments with $N_{D}=N_{K}=16$ and 32 are stuck. In panel 1(a) for $N_{D}=N_{K}=2$ and 4 the motion is in the anterograde (the kinesin motors win) direction and in panel 1(b) the filament motion for $N_{D}=N_{K}=2$ and 4 is in the retrograde direction (dynein motors win). Sometimes when there are more kinesin motors on the filaments, the direction of motion is dictated by dynein (see figure 6 panel 3(b) the green lines, figure 14 panels 1(b) and 2(b) and the supplemental text). As the stiffness increases, the fluctuations due to the stochastic effects increase (figure 14 panels 2(a) and 2(b) causing the slightly

245 stronger dynein to change the direction. In all the simulations except for the cases where both off rates are exponential, the competition between dynein and kinesin on the nodes was quickly resolved and the number of dynein and kinesin remains relatively stable for most of the time duration of the simulations.

Whenever one or both of the off rates are catch bond the initial conditions do not affect the qualitative features of the system (see figures 15 17. figures 20,22 . and figures 2527 in the supplemental data). Overall, whenever there is at least one catch bond off rate the dynamics are simpler.

\subsubsection{Both off rates exponential gives a rich variety of motion}

When both off rates are exponential the filament properties play an important role as can be seen in figure 7. There are two important results shown in this figure. The first is that the initial number of dynein motors determines the final states. If the initial number of dynein motors is high in the initial conditions (panels 2(a), 2(b), 4(a), and 4(b)), the number of dynein motors tends to stay high and if it is low (panels 1(a), 2(a), 3(a), and 3(b)) there are more kinesin motors than dynein motors for filaments with $N_{D}=N_{K}=16$ and 32 . The stiffest filaments (panels (c)) have few total motor molecules attached and most are still changing with time but there are more kinesin than dynein. 


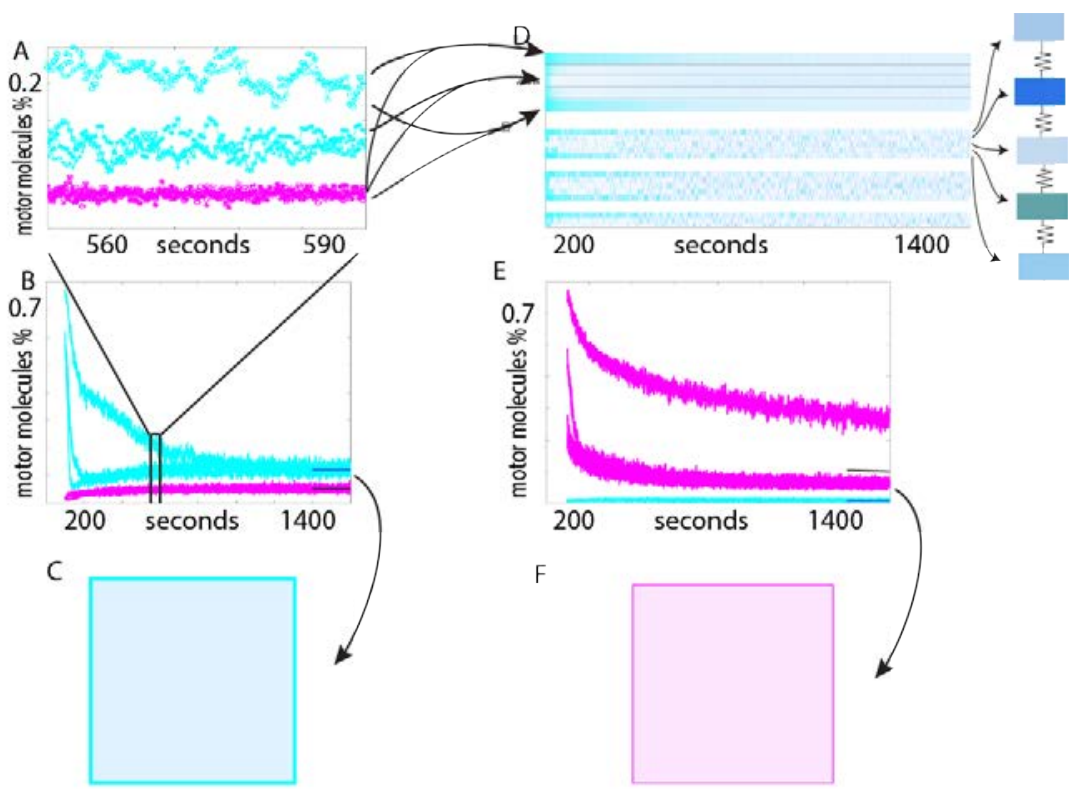

Figure 4: Explanation of the types of graphs used to depict the simulation results and how they relate to each other. Cyan (magenta) represents dynein (kinesin). Panels (B) and (E) show the average normalized number of dynein and kinesin for each filament node as a function of time. Here, filaments of length $N=5$ are considered. Panel (A) is a blow up of the boxed region in panel (B). In panel (A) the five cyan lines are distinguishable, one for each node on the filament. The 5 magenta lines are too close to distinguish. The combination of the cyan and magenta lines for each node gives the figure results for the top part of panel (D). In (D), each strip is formed from five color strips stacked vertically each depicting the number, as it changes in time, of motors attached to an individual node. The top is the average of all the simulations and is shown as a wider strip; whereas, the strips below are individual realizations. The arrows from panel (A) (only 3 pairing of the 5 are shown, namely, for node \#1, \#3 and \#5) depict how the lines in panel (A) give information for the strips in panel (D). In panel (D) the gray lines separating the nodes of the average filament are visualized only in this figure. For the lower strips in (D), the number of motor molecules for each node in a fiber and how it changed in time is indicated by color blocks for individual realizations. Panels (C) and $(\mathrm{F})$ show the final average number of motor molecules for the filament as determined by the least squares fitted black (for kinesin) and blue (for dynein) lines shown in (B) and (E). In (B) there are more dynein at the end of the simulation so the border in (C) is cyan. Since the lines are almost flat, the border in (C) is wider to depict that the number of motor molecules seems to be close to a "steady state". In (F) there are more kinesin so the border is magenta and the line is sloped, so the border is narrow indicating the system is not close to a steady state with respect to the number of motor molecules attached. In panel $(E)$, notice that the magenta curves have separated into two groups; the average normalized number of kinesin is not the same for all nodes. On interior nodes, on average, there are less kinesin attached than on the leading/exterior nodes. However, kinesin molecules are more abundant than the dynein molecules for each node. 

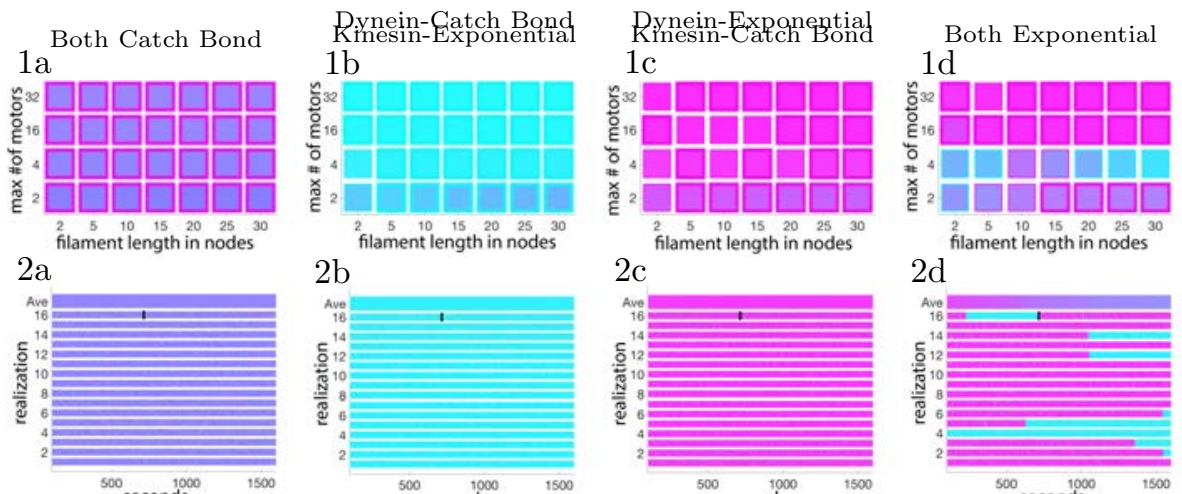
$2 \mathrm{~b}$

$2 \mathrm{c}$ $2 \mathrm{~d}$
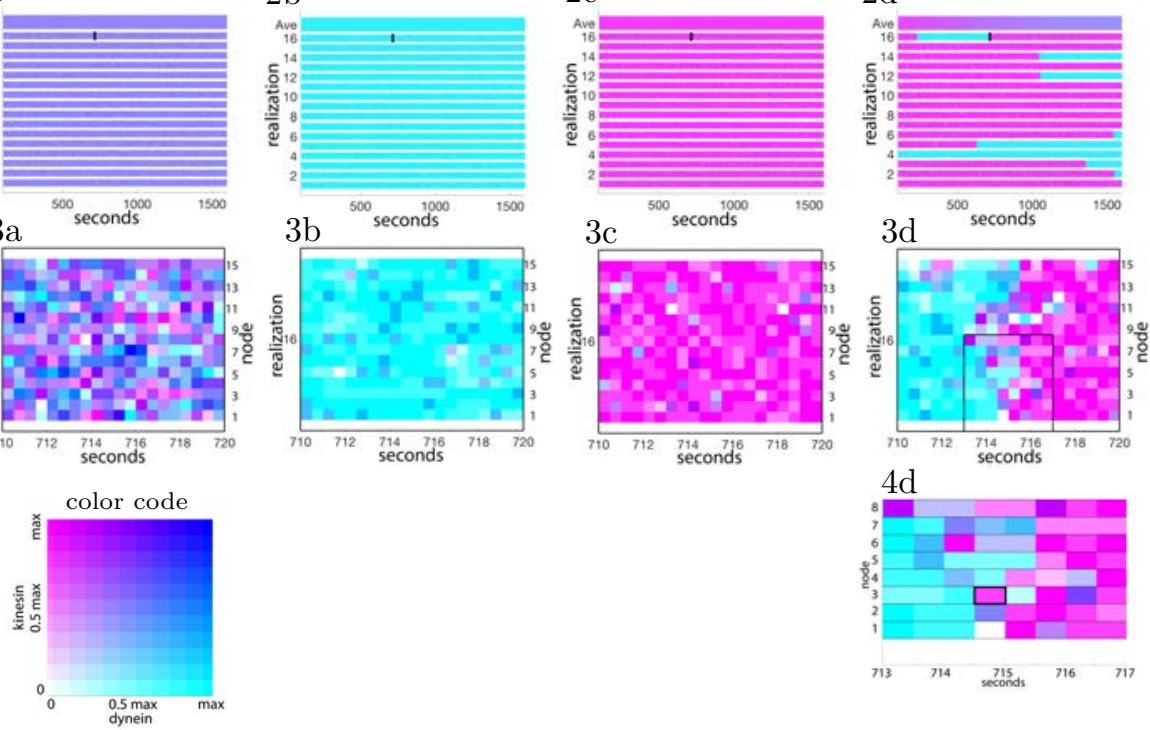

Figure 5: The time evolution and the final average number of motor molecules for simulations with different off rates. Panels (a) show simulations where both off rates are catch bond, panels (b) dynein catch bond and kinesin exponential, panels (c) dynein exponential and kinesin catch bond, and panels (d) both exponential. Panels 1 indicate the number of motor molecules averaged over all the nodes at the final time of the simulation 1600 seconds. The border color indicates which motor molecule is more abundant. If the border is thick it indicates the number of motor molecules is near a steady state value. If the border is thin the number of motor molecules is still changing. Panels 2 show 16 realizations and the average over all 100 realizations of the time evolution of the number of motor molecules. Panels 3 are blow ups of the small black rectangles in panels 2. Panel 4(d) is a blow up of the box in panel 3(d). The average of all the realizations is plotted twice as wide as each realization. The number of dynein and kinesin is color coded for each node and time. The nodes are plotted on top of each other thus motors attached to the first node are shown at the bottom of the horizontal strip of the realization and the motors on the last node are shown at the top of the color bar for each realization. The black horizontal lines in panel 4(d) separate the nodes. The black rectangle shows a solid color representing the number of motor molecules on one node (the third node) of the filament at one time. The color code is shown in the bottom left corner with cyan depicting dynein only, magenta kinesin only, and blue indicating equal amounts of each motor molecule. Note that the color key is normalized depending on the value of $N_{D}$ and $N_{K}$. All simulations had no motor molecules attached to the filaments as the initial conditions. The spring constants for the filaments in all the simulation is $\alpha$ which represents normal filament elasticity. In panels 2,3 , and 4 the fibers have 15 nodes and $N_{K}=N_{D}=4$. See figure 4 for a more detailed explanation of the visualization. 

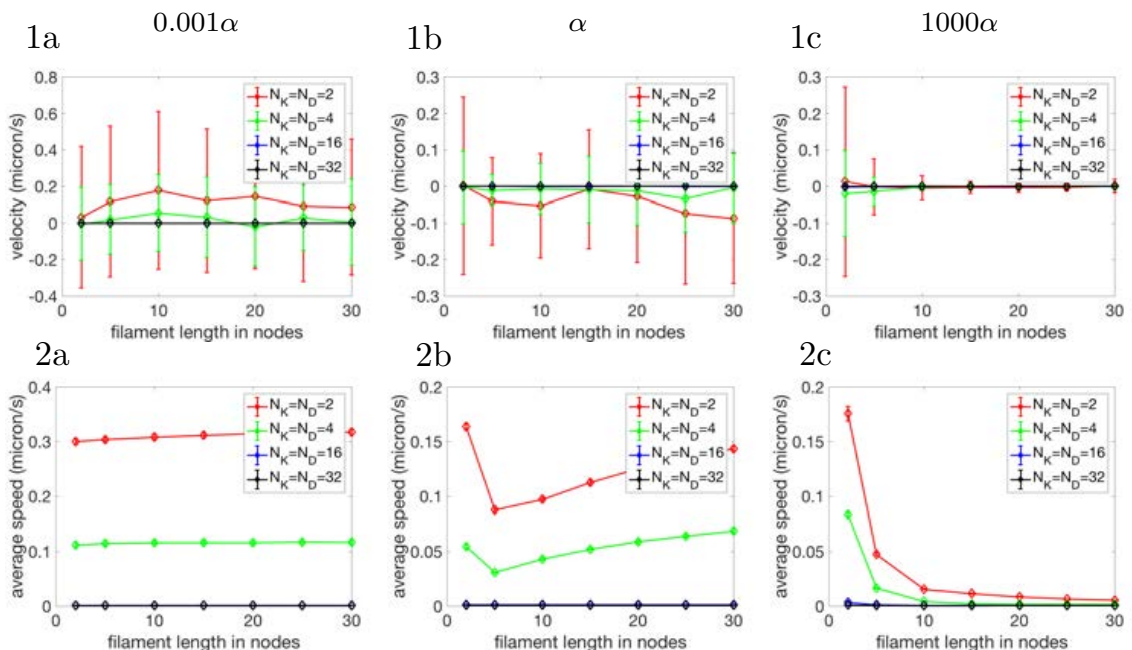

$2 \mathrm{~b}$
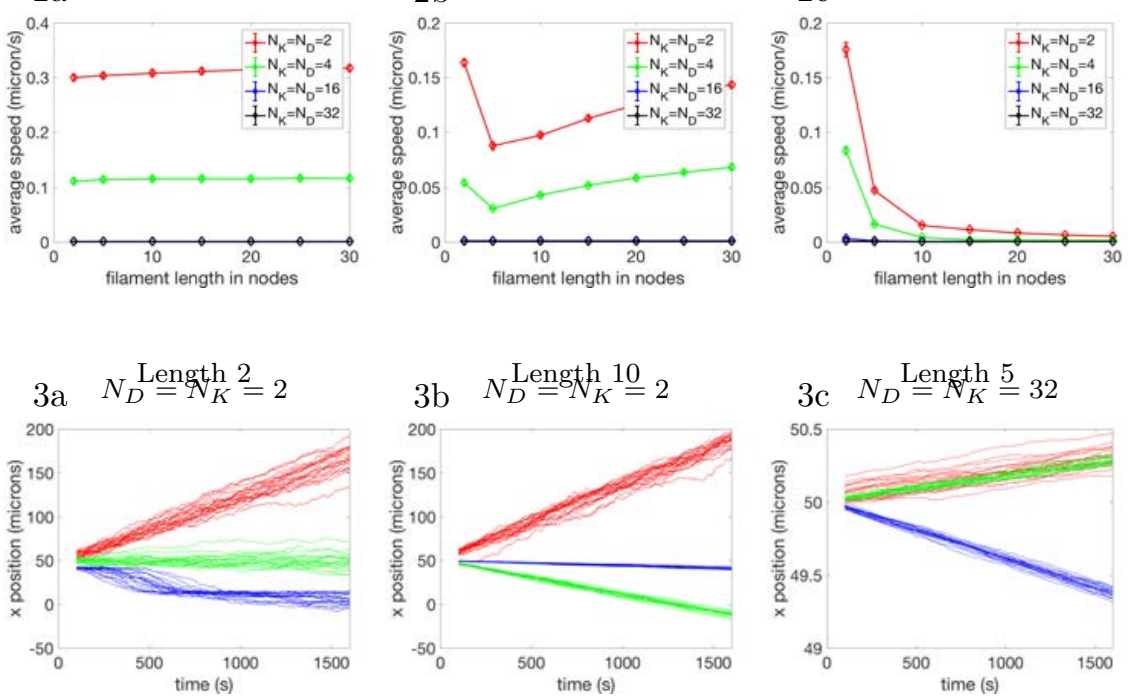

Figure 6: Velocity, speed and filament position when both off rates are catch bond. In panels 1 the expected instantaneous velocity of a filament is plotted as a function of filament length (when no forces are acting on a filament). In panels 2 the average speed is plotted. The error bars indicate the standard deviation. In panels $1(\mathrm{a})$ and $2(\mathrm{a})$ the filament elasticity is $0.001 \alpha$, in $1(\mathrm{~b})$ and 2(b), $\alpha$ and in $1(\mathrm{c})$ and $2(\mathrm{c}), 1000 \alpha$. The different curves within each frame are for constant $N_{D}=N_{K}$ with the values given in the legend. Panels 3 show the position of the first node as a function of time for selected realizations. The red lines are simulations of filaments with low spring constant, the green lines normal elasticity, and the blue lines high spring constant. Panel 3(a) is for simulations with filament length $N=2$ and $N_{D}=N_{K}=2$, panel 3(b) with filament length $N=10$ and $N_{D}=N_{K}=2$, and panel 3(c) with filaments of length $N=5$ and $N_{D}=N_{K}=32$. In panels 1 and 2,300 simulations are averaged for each data point. 
The second important result seen in figure 7 is that filament length and the maximum number of motor molecules can cause the filament to change directions (panels (b)). In panels 1(b) and 3(b) the bottom rows have filaments moving in both directions with most moving according to kinesin. By increasing the maximum number of motors allowed to 4 , the balance shifts to most fibers moving according to dynein. Increasing the maximum motors allowed again results in unidirectional motion dictated by kinesin. When both off rates are 270 exponential, a rich variety of motion can be obtained by changing the cellular context (initial conditions) and the filaments properties $\left(N_{D}=N_{K}\right)$. In this case the dynamics are more complex than the dynamics with other combinations of off rates.

\subsubsection{Homogeneous off rates allow direction switching}

When dynein and kinesin have the same off rates the simulations show that IFs switch directions, figure 8 panels 1 (both catch bond) and 4 (both exponential). The filaments alternate between positive and negative velocities with the period between switching increasing as the filament length increases. If the maximum number of motors is greater, the switching behavior disappears and if the maximum number is lower, the switching behavior can be seen with a high frequency. When the off rates are not the same there is no switching behavior seen (panels 2 and 3). Filament speeds are faster when there is no switching. In these cases one motor clearly dominates resulting in less competition and faster speeds. Filaments with low elasticity and normal elasticity change lengths with time as shown in Figure 9. The filaments with the smallest spring constant change length significantly whereas the filaments with normal elasticity change length less than 50 percent of their rest length and filaments with the highest spring constant remain about their rest length.

\subsection{Filament properties regulate motion efficiency}

The properties of filaments, their elasticity, length, and number of binding sites play a role in the global dynamics of filaments. Figure 10 shows the stiffest filaments have the slowest speeds. For low elasticity value the speed remains relatively independent of the filament length and for all but $N_{D}=N_{K}=2$ the same is true for normal elasticity. For the stiffest filaments, the speed is affected by the filament length. Interestingly, for most cases (varying length, maximum number of allowed motors, and off rates) the realistic value for the filament elasticity (the green lines in figure 8) give the most efficient motion.

In all the cases (varying length, elasticity, and off rates) the maximum number of motor molecules affects the speed. This is consistent with in vitro experiments of vesicles [35] but inconsistent with in vivo experiments of vesicles [36. Yet the in vivo experiments dealt with vesicles with one or two motors and considered only "periods of uninterrupted motion". Our results give the average speed over a long period with a much larger range of motor molecules. For all cases except both off rates catch bond the speed increase with increasing maximum number of motor molecules. This is due to the fact that when both 


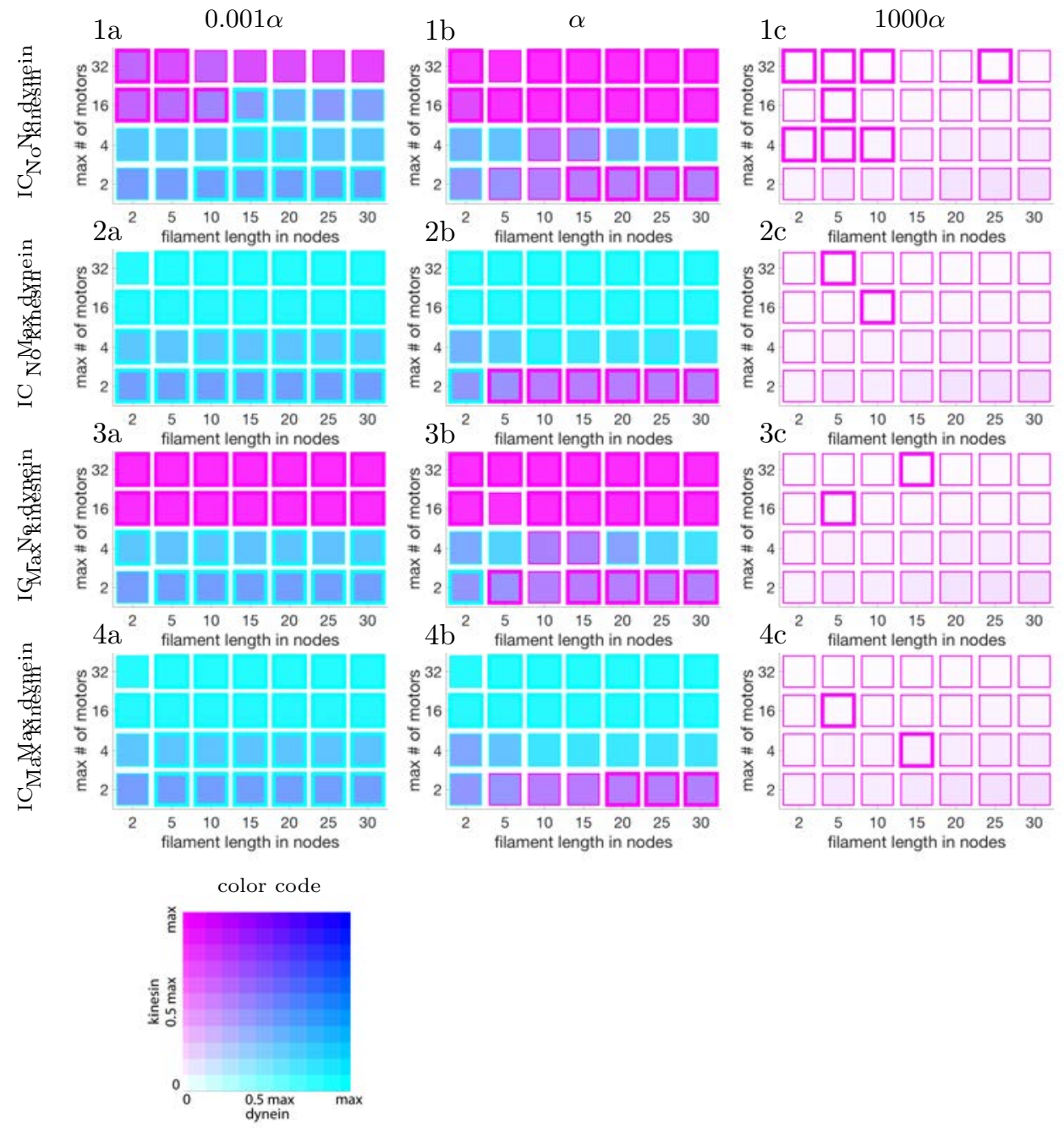

Figure 7: The final average number of motor molecules for simulations where both off rates are exponential with different initial conditions. Observe that panels $1(\mathrm{a})$ and $1(\mathrm{~b})$ are similar to $3(\mathrm{a})$ and 3(b) and 2(a) and 2(b) are similar to 4(a) and 4(b). Note that in panel 1(b) where $N_{D}=N_{K}=4$, if there are filaments of all lengths, filaments are traveling in both directions or bidirectional transport. When $N_{D}=N_{K}$ is changed up or down the transport becomes more directed. The graphs show the final number of dynein and kinesin averaged over realizations and nodes. The border color indicates which motor molecule is more abundant. If the border is thick it indicates the number of motor molecules is near a steady state value. If the border is thin the number of motor molecules is still changing. The initial conditions change for each row and the filament elasticity varies from low to high with the columns. Panels 1 have no motor molecules initially attached, panels 2 have maximum dynein attached and no kinesin, panels 3 have maximum kinesin and no dynein, and panels 4 have maximum of both motor molecules initially attached. The color key is shown in the bottom left. Cyan depicts dynein only, magenta kinesin only, and blue means equal amounts of each motor molecule. The time shown is 1600 seconds. 

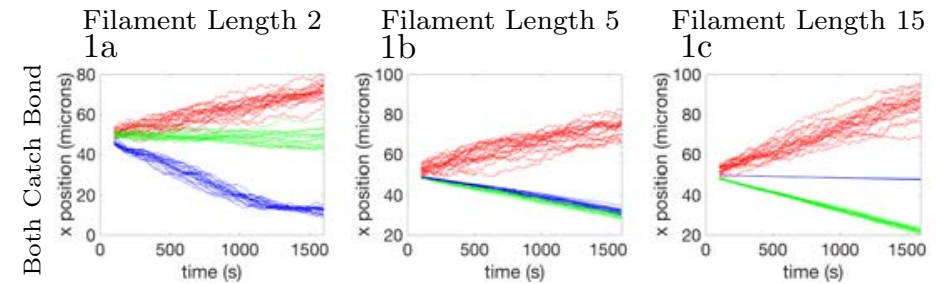

Filament Length 25
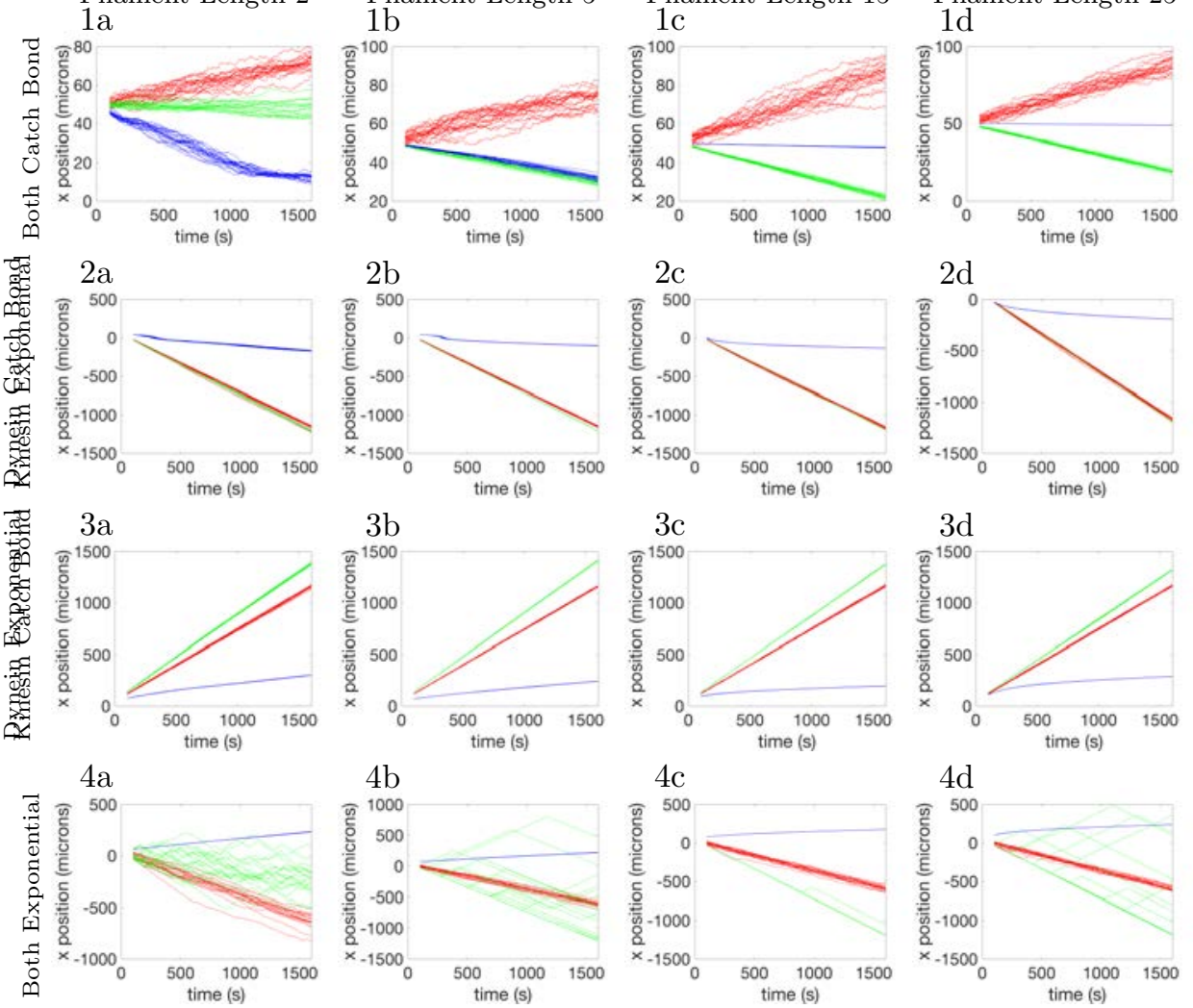

Figure 8: Time plots of the location of the first node on selected filaments for all combinations of off rates. The red lines are simulations of filaments with low spring constant $10^{-3} \alpha$, the green lines normal elasticity $\alpha$, and the blue lines high spring constant $10^{3} \alpha$. Panels (a) show results for simulations with filament length $N=2$, panels (b) have $N=5$, panels (c) have $N=15$, and panels (d) have $N=25$. Panels 1 show off rates both catch bond, panels 2 have dynein catch bond, kinesin exponential, panels 3 have dynein exponential, kinesin catch bond, and panels 4 have both exponential. All simulations have $N_{D}=N_{K}=4$ and initial conditions with the maximum number of dynein and no kinesin attached. 

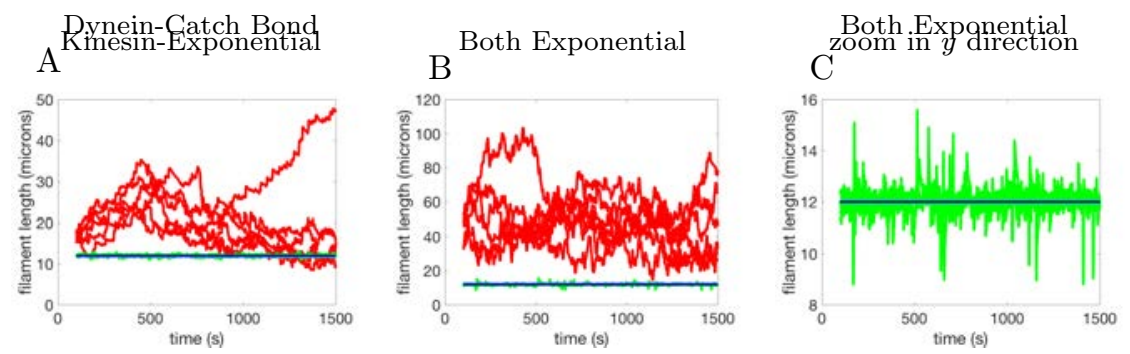

Figure 9: Time plots showing how the filament length changes due to the force acting on the filament. The length is given in microns for selected filaments (compare with figure 8 panels $2(d)$ and $4(d)$ ) and changes due to the elasticity of the filaments. The red lines are simulations of filaments with low spring constant $10^{-3} \alpha$, the green lines normal elasticity $\alpha$, and the blue lines high spring constant $10^{3} \alpha$. All simulations have $N_{D}=N_{K}=4, N=25$ or filaments of length $12 \mu \mathrm{m}$ initially, and initial conditions with the maximum number of dynein and no kinesin attached.

off rates are catch bond the competition between motor molecules results in filaments with a mixed number of dynein and kinesin. So neither molecule is as dominant as in the cases with other off rate combinations, thus allowing more motor molecules does not increase the speed. In fact it decreases it due to fewer stochastic effects.

\subsubsection{Elasticity and number of motors affect node coordination}

To understand how the number of motors allowed and filament elasticity play a role on the global dynamics, the study now focuses on the local dynamics at each node along a fiber. Even though the dynamics of the motor molecules are local to each node, in many simulations the number of dynein and kinesin become coordinated for each node on a filament. Since in the case with both exponential off rates there is more competition between the motor type the phenomenon is more easily seen. Figure 11 shows how the maximum number of motor molecules and the filament elasticity affect the coordination of nodes along a fiber in the case of both exponential off rates. Increasing the maximum number of motor molecules (going down the columns) increases the coordination of the nodes. The filament elasticity has a unimodal behavior for node coordination with the greatest coordination at the normal filament elasticity (panels 2(b), $3(\mathrm{~b})$, and 4(b)). The weakest filament elasticity has the least coordination of nodes (panels 1(a) and 2(a)). For the stiffest filaments (panels (c)) there are not many motors attached and the proportion of motor molecules on each node seems to change less than the overall number of motor molecules. Notice in panel 1(a) there is not much coordination of nodes within a filament. In panel 1(b) there is more coordination of the nodes even though over time the nodes switch from having more dynein to kinesin and back. It is interesting to see how the transition from one type of motor molecule to the other is so abrupt in panel 2(b). In panel 3(a) the transition is more slow but eventually all the nodes in the filament transition from having more of one molecule to having 

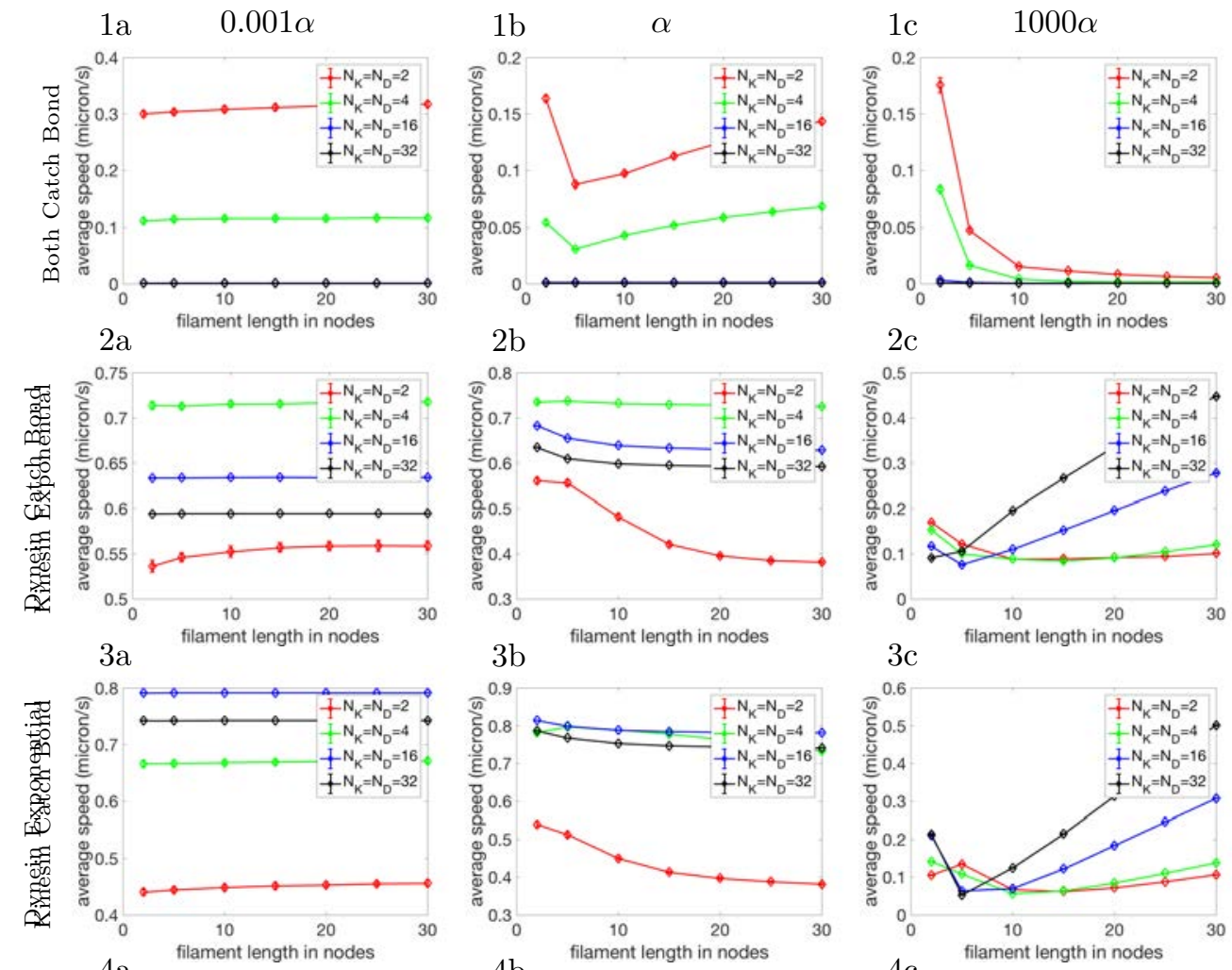

$3 \mathrm{~b}$
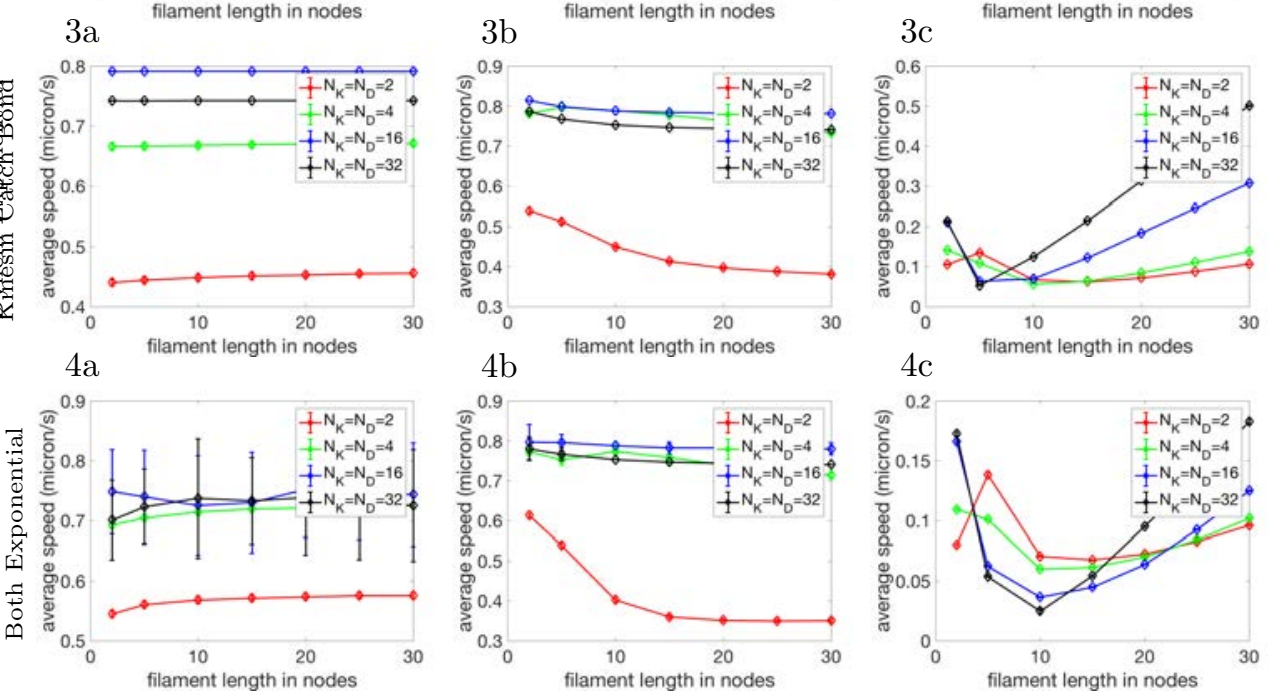

Figure 10: The expected speed of a filament is plotted as a function of filament length for all combinations of off rates. The off rates change from row to row and the filament elasticity varies from low to high with the columns. Panels 1 have both off rates catch bond, panels 2 have dynein catch bond and kinesin exponential, panels 3 have dynein exponential and kinesin catch bond, and in panels 4 both are exponential. Panels (a) have filament elasticity $0.001 \alpha$, panels (b) have $\alpha$, and panels (c) have $1000 \alpha$. The different plots within each frame are for constant $N_{D}=N_{K}$ with the values given in the legend. All simulations have no motor molecules attached as initial conditions. The error bars indicate the standard deviation. 
more of the other and in panel 4(a) the transition is even slower. It appears the transition from one motor type to the other is faster as $N_{D}=N_{K}$ decreases and as the elasticity increases. The coordination of nodes is due either to the elastic coupling, this behaviour is common in coupled dynamical systems, or the maximal number of motor types allowed per node on filaments. If there are more motors of a given type on a node it should exert stronger forces on neighboring 340 nodes. When the nodes within a filament are coordinated one would expect greater filament speeds which is observed in figure 10.

\subsubsection{Filament elasticity is optimized for fast filament motion}

To further understand the relationship between speed and the filament elasticity we ran simulations with several different elasticity parameters when a type of motors dominates. Following biological observations [37, 29, 16, 30, 14, a catch bond off rate is chosen for dynein whereas kinesin is assumed to have an exponential off rate. Here we are calculating the overall speed of filaments as the absolute value of the final filament position minus the initial position divided by the total time (notice this is not the same as the speed shown elsewhere). The results are shown in figure 12. The most efficient motion lies near the peaks in figure 12. The greatest speeds are achieved for values of filament elasticity near the realistic range. It is interesting to note that the elasticity which gives the greatest speed for $N_{K}=N_{D}=2$ varies with filament length and seems to be tuned for shorter filaments. Likewise for $N_{K}=N_{D}=4$ the realistic 355 system parameters are set for the greatest speeds. Larger numbers of motors $N_{K}=N_{D}=16$ and 32 allow filaments having stiffness $10 \alpha$ to be transported in the most efficient way.

\section{Discussion}

Transport of IFs in cells by antagonistic motors proteins along microtubules is now well documented [38, 7, 6; ; however, its regulation is still not well understood. Here, using a mathematical model to overcome experimental intractability, different features of this transport are investigated. We have developed and explored the behavior of a model for the transport of IFs which can also be applied to coupled cargo transport. A summary of our results is shown in table 1 .

Four limiting assumptions of the model are load sharing, the computational treatment of binding independence, motor competition, and Hookean elasticity for IF. The model assumes that motors of each type equally share the load per node. When the motors are crowded this is unlikely to be the case [39], but 370 in our system even when $N_{K}=N_{D}=32$ the motors should not be crowded on a filament due to the node spacing. For computational convenience, in the numerical algorithm we assume binding events on each node are independent of binding events on other nodes. We believe the force dependence of the binding is the primary feature and including the full independence of bindings is secondary. We assume that motors only compete with motors of the same type but they 

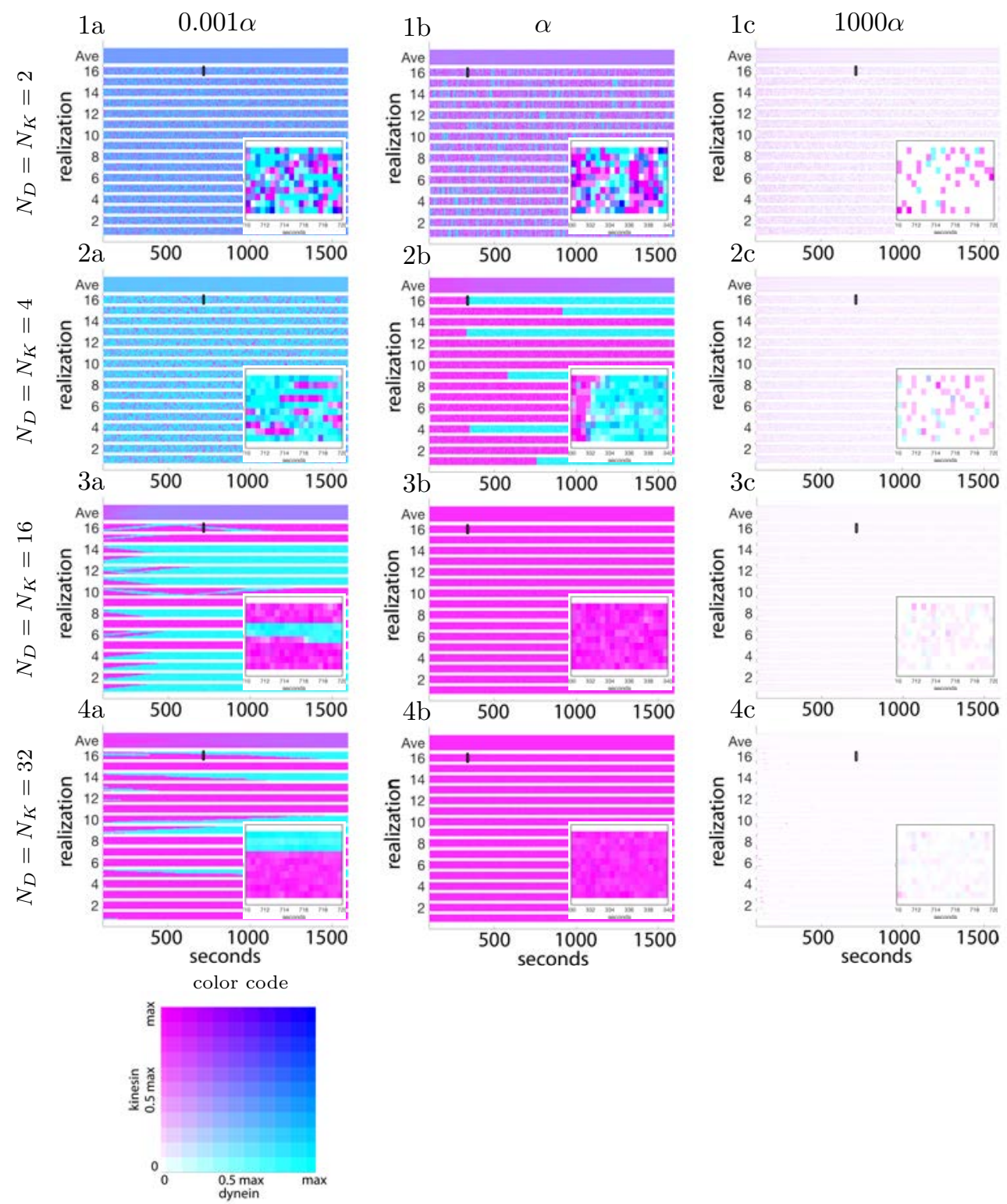

Figure 11: Variation of dynein and kinesin over time at each node for selected filaments when both off rates are exponential. Each panel shows several realizations for the same simulation. Observe how nodes within a fiber become correlated as you move down the rows or towards the center column. The least coordinated fibers are in panels $1(\mathrm{a})$ and $2(\mathrm{a})$ and the most coordinated are in panels 2(b), 3(b), and 4(b). Each column has a fixed elasticity for the filaments going from left to right $0.001 \alpha, \alpha$, and $1000 \alpha$. Each row has fixed $N_{D}=N_{K}$ going from top to bottom $2,4,16$, and 32 . The insets are blow ups of the small black rectangle in each panel. All the simulations have filament length 10 nodes, and no motor molecules attached as the initial conditions. The color key is in the bottom left. 

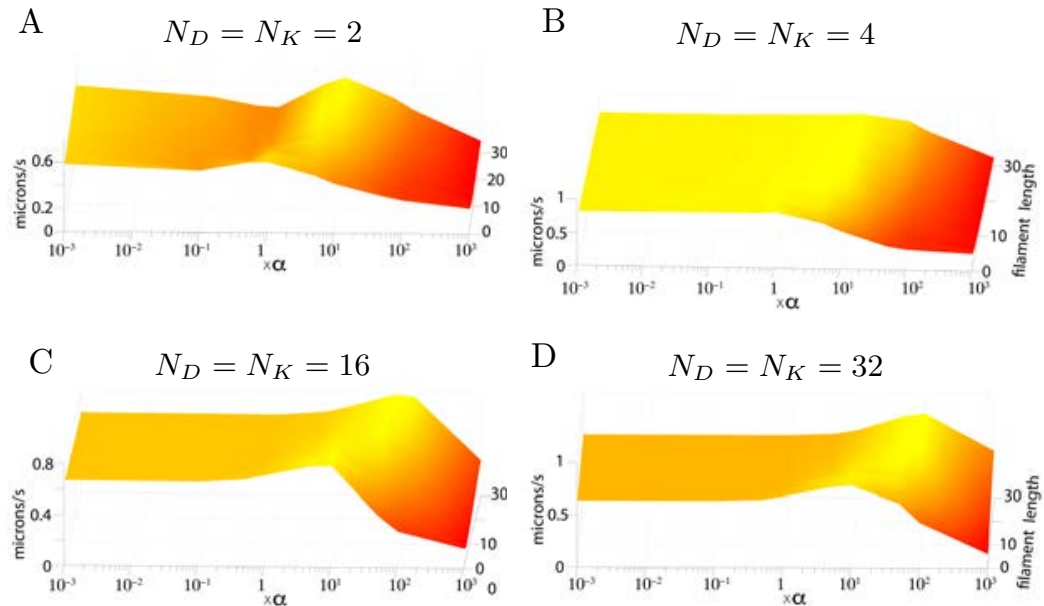

Figure 12: Surfaces of the filament overall speed are shown for changes in filament length and elasticity as a factor of $\alpha$. In these simulatons the dynein off rate is catch bond and kinesin off rate is exponential. The axis for filament elasticity is logarithmic. The maximum speeds of the surfaces are $0.64,0.75,0.79$, and 0.80 for panels A-D respectively. Simulations for elasticity values of $.001 \alpha, .1 \alpha, .5 \alpha, \alpha, 5 \alpha, 10 \alpha, 50 \alpha, 100 \alpha$ and $1000 \alpha$ are used to create the surfaces. In these simulations no motor proteins are initially attached to the filaments. The overall speed is calculated as the average, over all the filaments, of the absolute value of final position minus the initial position divided by the overall time. The color code indicates the speed as does the height. The filament length is measured in nodes. 
Table 1: Summary of Model Results

kinesin exponential kinesin catch bond kinesin exponential kinesin catch bond dynein exponential dynein exponential dynein catch bond dynein catch bond

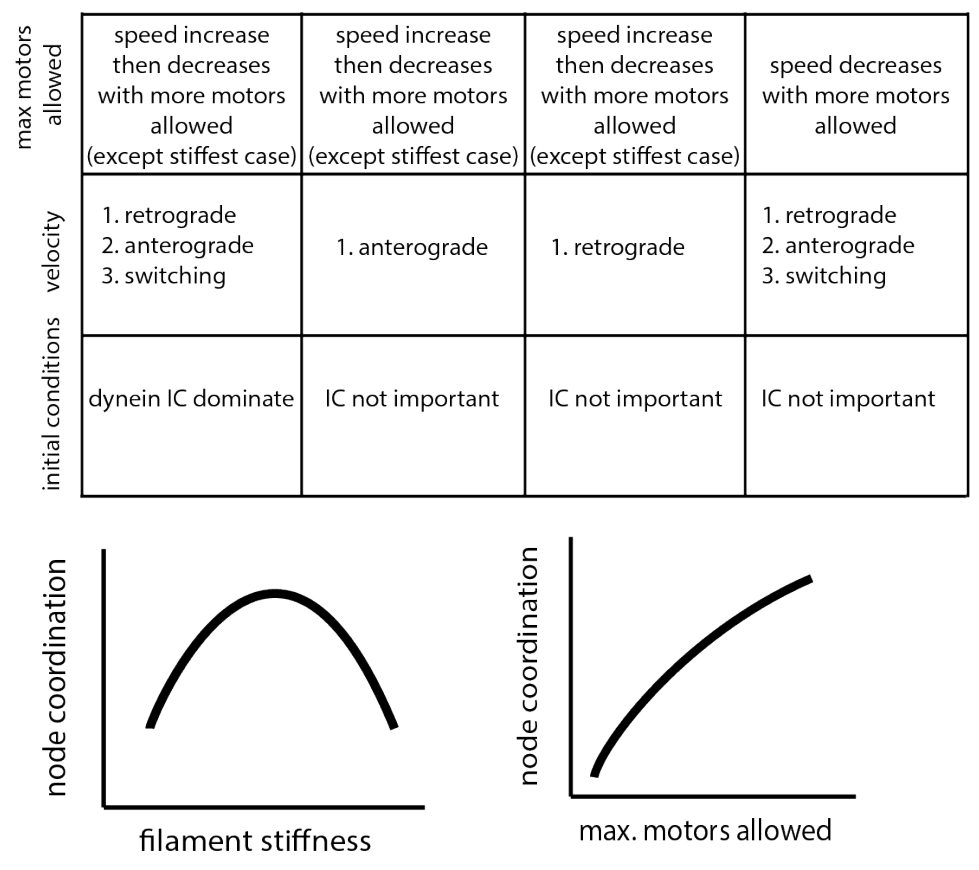

Filament transport can be optimized with changes in elasticity

* Stiff filaments have few motors on interior nodes and are transported mainly by motors at the ends

Change in the maximum number of motors allowed (some cases) changes filament from retrograde to anterograde motion 
likely compete with both types of motors due to space constraints. Several simulations with competition between motors (data not shown) indicate that the qualitative features of the system do not change. Here a linear elastic response is assumed for IFs. In the next steps of the work, the effects of nonlinear response will be investigated [34.

The purpose of this theoretical study is to better understand how motor and filament properties affect the transport of IFs. The model will certainly improve as we learn more about the motor properties. As for now, even for kinesin, which is well studied and better understood than dynein, conflicting 385 information is available. For instance, the off rate measured in 14 seems to be catch bond, it may be an artifact of the small number of data points or the experimental technique. More recently the off rate for kinesin has been measured as an asymmetric function where the assisting loads have greater detach rates than opposing loads [4, 41. In these studies $F_{d}$ has been estimated to closer to five. Yet in other recent modeling $F_{d}=4$ works well 42. These facts highlight the need for this type of theoretical study. Understanding how the qualitative features of the motors affect the transport is important. It is the relative strength between the two motors which seems to determine most of the behavior.

It is interesting that the more complicated dynamics correspond to the case where both off rates are exponential. This gives the cell more options on controlling the behavior of the filament motion. The model indicates that when one of the off rates is catch bond the dynamics are more stable and for the cell to alter the motion of the IFs more fundamental alterations to the system must be employed.

Our results suggest a possible mechanism for cells to switch from bidirectional transport to directionally biased transport towards the cell membrane as seen in 2. In the case where both off rates are exponential, low initial dynein, normal filament elasticity, and a maximum of 4 motor proteins per node (fig405 ure 7 panels $1(\mathrm{~b})$ and 3(b)), filaments are moving in both directions depending on their length. The short and long fibers are moving towards, the minus end of the microtubules, the cell center and the intermediate length filaments are moving towards the plus end of the microtubule. If the maximum number of motor molecules per node is increased from 4 to 16 (or decreasing from 4 to 2 ) ${ }_{410}$ then all (all but the shortest) filament lengths move toward the plus end of the microtubule (to the cell periphery). Thus according to this model one method for the cell to switch from bidirectional transport to transport biased towards the end of the microtubule would be to regulate the number of binding sites for the motors on the filaments. IF's affinities and interactions with motors and the ${ }_{415}$ number of binding sites could be regulated by post-translational modifications which affect IF's 2, 43, 44.

These same results of the model suggest that filaments could be sorted by length in the cell. Thus shorter filaments could accumulate at the cell center while intermediate lengths would accumulate at the periphery of the cell. This 420 could have implications on how the severing and annealing process [45, 6] works. One could expect more annealing to occur in regions of the cell with high con- 
centrations of shorter filaments. The new intermediate length filaments would then be transported out of that region.

Catch bond off rates increases the ability of the motor to dominate the system. For the parameters used here when both motors have catch bond off rates kinesin dominates but not by much. When the off rates are mixed, catch bond and exponential, the catch bond motor dominates. Of course by altering the parameters one could find a region where the motors are more evenly matched. Although the parameters for kinesin- 1 are well established, the parameters for 430 dynein are less well known [46, 47]. Yet understanding them will be essential to any modeling efforts and to a further understanding of the system.

We found that the initial number of motors available to a filament and filament properties only influence the final state of the system when the off rates for both dynein and kinesin are assumed to be exponential (figure 7). In all other cases the initial number of motor molecules attached to the filament did not affect the long term dynamics of the system. We believe it is more likely that the cell changes the properties of cargoes or filaments (e.g. by posttranslational modifications) as opposed to the initial state of the system. If most of the filaments are not associated with microtubules, the cell may be able 440 to preload the filaments with motor proteins in an attempt to alter the initial conditions. Yet in the case with both off rates as exponential, it seems the initial number of dynein attached to the filament determines what the dynamics will do.

It appears two conditions are necessary for filaments to exhibit switching behavior: low maximum motors and evenly matched motors as determined by the motor properties. Switching is only seen when there are low (2 or 4) maximum motors allowed. The stochastic behavior of the system allows nodes with few motors to randomly switch when the motors are more evenly match. This is consistent with most of the tests in [14, where the fewer number of motors 450 gave the most percentage of quick reversals. If there are many motors associated with a node it will be less likely to switch. This is also consistent with the node coordination within a fiber when there are more motors allowed. Additionally, when one motor strongly dominates no switching behavior is seen but when the motors are more evenly matched (in the case of both catch bond or 455 both exponential) switching behavior can be seen. Under these circumstances one would expect most filaments to be stalled (or moving very slowly) due to the competition between motors. There would be a few filaments where the stochastic effects allow one motor to dominate resulting in switching behavior

For all off rate combinations, the coordination of motor proteins between different nodes of the same filament (or coupled cargo) increases with more motors allowed. The coordination between nodes has a unimodal behavior as a function of the fiber elasticity. For normal elasticity the nodes within a filament are more coordinated. The increased coordination as the coupling increases is expected but a maximum is not. For the very stiff fibers the forces cause the 465 motor molecules to detach giving a lower overall density of attached motors. This is due to the assumption of load sharing per node. It is interesting to note that it is optimal for an elastic filament to have motors at all the nodes, but 
for a stiff fiber, motors on the end nodes and fewer motors in the middle seems more stable (figure 11 panels $1(\mathrm{a})$ and $1(\mathrm{c})$ ). Thus the stiff filament is carried by the end nodes. This may be due to the fact that the end nodes have only one spring attached to them and do not have the same back and forth pull as the interior nodes.

The coordination of nodes helps explain why the same two parameters: maximum motors allowed and elasticity are also relevant to the fiber's average speed 475 and instantaneous velocity. More coordination between nodes should result in more efficient transport. The most efficient transportation occurs for the realistic value of filament elasticity when the maximum number of motor molecules is either 2 or 4 per node. The system seems to be tuned for efficient transport when there are only a few motors available for each node $\left(N_{K}=N_{D}=2\right.$ or 4$)$. Since the internode distance in our simulations is $0.5 \mu \mathrm{m}$ which corresponds to about 12 unit-length-filaments (ULF's) this indicates that there would be only one motor of each type for several ULF's. As expected, varying the internode distance did not significantly impact the results. Adding more nodes and keeping the Young's modulus constant does not affect the elastic properties of the 485 filament. It only affects the distribution of the attached motor molecules. From a force standpoint this should not alter the results of our simulations. Numerical simulations verified this (see Supplemental Data 7.5).

The two other cytoskeletal components, actin and microtubules are up to 10 to more than 1000 times stiffer than IFs [4]. Our model predicts that these fibers would not be transported as efficiently. The actin network is extremely dynamic and there are no reports that the filaments are transported along microtubules although some myosin motors can bind. Microtubules have been reported to be transported along microtubules at fast speeds of 1 micron per second [49] and at slow speeds of about 4 microns per minute [50]. In the first study the average length of the microtubules is 2.7 microns and the authors state the motion is infrequent and highly asynchronous. In the second study the filament lengths are not reported and velocities are mean peak velocities. They also reported that many segments spent most of the time not moving. Our model is more in agreement with the second study and predicts that stiffer 500 filaments will be transported more slowly. Yet the first study may also be predicted by our model. Figure 10 shows that the speeds for stiff filaments vary depending on the motor dynamics, the filament length and the number of maximum motors attached. Thus short filaments with the correct motor loading and dynamics can be faster.

\section{Methods}

\subsection{Modeling The Filament}

The intermediate filament is modelled as a series of $N$ nodes connected by $N-1$ springs. IFs are elastic and can stretch up to three times their length [51, 34. The rest length of the springs between the nodes is denoted $\ell$ and is ${ }_{510}$ fixed at $0.5 \mu \mathrm{m}$. Vimentin filaments are made up of the longitudinal annealing 
of unit-length-filaments, ULFs [52]. Each ULF is composed of about 8 tetramers laterally assembled forming the subunit of IFs. Each ULF contributes about $0.04 \mu \mathrm{m}$ to the length of an intermediate filament [53] thus the internode spacing in the model is about 12 ULFs which is about the persistence length of vimentin 515 [54, 55]. We assume that dynein and kinesin bind at a low density per unit length to IFs. To our knowledge imaging has indicated that there are not high densities of these molecules co-localized to IFs [56, 57.

The number of binding sites available on internode length is $N_{D}$ for dyneins and $N_{K}$ for kinesins. Yet at any given time $n_{D}$ is the number of dynein actually ${ }_{520}$ bound to the node and similarly $n_{k}$ is the number of kinesin. In the model, the motors randomly bind to filament nodes, which represent a portion of the filaments. The details of how IFs and motor molecules associate are not yet fully understood. Thus allowing the motors to randomly bind to the filaments is a reasonable modeling strategy which can be changed as more information becomes available. When a node is bound to dyneins and/or kinesins its velocity is determined by the speed of the motor molecules which is a function of force. Motors of the same type share the load equally, i.e., we use a mean-field load sharing model similar to the model in [13. If there are no motor molecules bound to a node it moves according to a force balance equation assuming low 530 Reynold's number. Figure 2 shows a schematic of the model.

The model assumes the nodes exert forces on each other according to Hooke's law. The force is proportional to the distance between connected nodes. The drag coefficient for the nodes is calculated assuming the nodes are cylinders in a fluid flowing parallel to the axis of the cylinder [58. The drag is modeled 535 assuming a low Reynold's number and is proportional to the velocity of the nodes. We denote the location of the nodes as $x_{i}$, a point in $\mathbb{R}$ where $i$ ranges from 1 to $N$.

The mathematical model is force based and uses Newton's second law of motion. Due to low Reynold's number the acceleration term can be ignored and the resulting equations are first order. The equations of motion for the location of a node are given by

$$
\mu \frac{d x_{i}}{d t}=\underbrace{n_{D, i} F_{D, i}}_{\text {forces due to dyneins }}+\underbrace{n_{K, i} F_{K, i}}_{\text {forces due to kinesins }}+\underbrace{F_{r e s, i}}_{\text {forces due to filament elastic property }}
$$

where $n_{D, i}$ (resp. $n_{K, i}$ ) is the number of dyneins (resp. kinesin) attached to the node $i, F_{D, i}$ (resp. $F_{K, i}$ ) is the force acting on a single dynein (resp. kinesin) attached to node $i$, and $\mu$ is the drag coefficient. All nodes except the two end nodes $\left(x_{1}\right.$ and $x_{N}$ ) are connected to two neighboring nodes (see figure 2) and thus have two forces acting on them due to the elastic filament properties. The forces are proportional to the distance between the connected nodes minus the rest length of the spring. For the two end nodes there is only one force. The 
force equation due to the elastic property of the filament is given by

$$
F_{r e s, i}=-\alpha\left(\left(\left|\left(x_{i}-x_{i-1}\right)\right|-\ell\right) \frac{x_{i}-x_{i-1}}{\left|x_{i}-x_{i-1}\right|}+\left(\left|\left(x_{i}-x_{i+1}\right)\right|-\ell\right) \frac{x_{i}-x_{i+1}}{\left|x_{i}-x_{i+1}\right|}\right)
$$

where $i=2, \cdots N-1, \alpha$ is the spring constant, and $\ell$ is the rest length of the spring ( in our case $\ell=0.5 \mu \mathrm{m}$ ). Similarly for $i=1$ the equation is

$$
F_{r e s, 1}=-\alpha\left(\left(\left|\left(x_{1}-x_{2}\right)\right|-\ell\right) \frac{x_{1}-x_{2}}{\left|x_{1}-x_{2}\right|}\right)
$$

and for $i=N$ the equation is

$$
F_{r e s, N}=-\alpha\left(\left(\left|\left(x_{N}-x_{N-1}\right)\right|-\ell\right) \frac{x_{N}-x_{N-1}}{\left|x_{N}-x_{N-1}\right|}\right) .
$$

The forces due to the dynein and kinesin are determined by the stochastic nature of the binding of the motor molecules, the magnitude of the forces depends on the number of transport molecules associated with the node.

\subsection{The Forces Due to Motor Molecules}

We model the motion of nodes attached to motor molecules, using a meanfield load sharing approach, in manner similar to the model developed by [13] with two modifications. The first modification is to allow for cooperative forces acting on motor molecules and the second modification is to consider the drag force on a node. The first modification is necessary due to the coupling of the nodes. Each node has forces due to coupling with neighboring nodes which can either oppose or assist the motors moving the node. The second modification makes the model more realistic for a long fiber where the drag may play a more important role than for a single cargo. As in Muller, we assume the number of forward moving motors (kinesin) bound to a node is $n_{K}$ which stochastically changes and likewise $n_{D}$ is the number of bound backward moving motors (dynein). There is a tug-of-war between the motors to determine the motion of the node. We, as do they, allow for an external force opposing the motion of the node and define the velocity of the motors by a piecewise linear function of force. In Muller, the domain of the velocity functions is $[0, \infty)$, whereas in our model the domain is $(-\infty, \infty)$. Both our model and their model assume that opposing motors act as loads and identical motors share the load. We depart from their notation allowing both positive and negative forces with the positive $x$ direction corresponding to the plus direction of the microtubule. Let $F_{s K}$ be the stall force of kinesin which is negative. Then the velocity function for kinesin is given by

$$
v_{K}\left(F_{K, i}\right)=\left\{\begin{array}{lc}
v_{b K}\left(1-F_{K, i} / F_{s K}\right) & F_{K, i} \leq F_{s K} \\
v_{f K}\left(1-F_{K, i} / F_{s K}\right) & F_{s K}<F_{K, i}<0 \\
v_{f K} & 0 \leq F_{K, i}
\end{array}\right.
$$



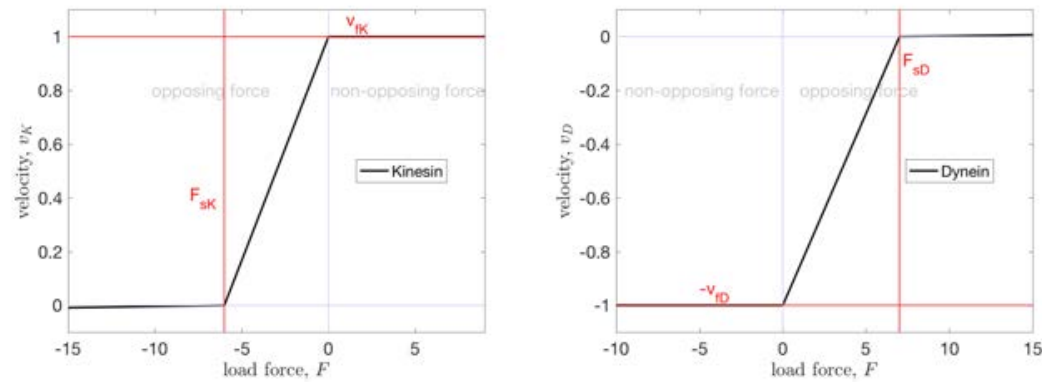

Figure 13: The functional form of the velocity of kinesin and dynein as a function of load force, Eq. (5) and Eq. 6). Notice that the line in the left panel for $F<F_{s K}$ has a slight slope as does the line in the right panel for $F>F_{s D}$.

where $v_{f K}$ and $v_{b K}$ are positive constants giving the forward and backward speed of kinesin. Let the stall force for dynein be $F_{s D}$ which is positive. The velocity function for dynein is

$$
v_{D}\left(F_{D, i}\right)=\left\{\begin{array}{lc}
-v_{f D} & F_{D, i} \leq 0 \\
-v_{f D}\left(1-F_{D, i} / F_{s D}\right) & 0<F_{D, i}<F_{s D} \\
-v_{b D}\left(1-F_{D, i} / F_{s D}\right) & F_{s D} \leq F_{D, i}
\end{array}\right.
$$

where $v_{f D}$ and $v_{b D}$ are positive constant representing the forward and backward speed of dynein.

Consider node $i$. The drag force is $-\mu v_{i}$ where $v_{i}$ is the velocity of the node. The motor molecules adjust the force to maintain the velocity relation. The force balance equation is

$$
-n_{K, i} F_{K, i}-n_{D, i} F_{D, i}+F_{r e s, i}-\mu v_{i}=0 .
$$

The maximum force generated by each kinesin molecule is $-F_{s K}$ and similarly for a dynein molecule. If $-n_{K, i} F_{s K}-n_{D, i} F_{s D}+F_{r e s, i} \geq 0$ kinesin will win the tug-of-war and node $i$ will move to the right.

All the motor molecules associated with a node move at the same velocity as the node. Thus to find the velocity of the node, $v_{i}$, the velocities of the motor molecules defined by equations (5) and (6) are equated, i.e. $v_{K}\left(F_{K, i}\right)=$ $v_{D}\left(F_{D, i}\right)$. Using the force balance equation, equation 7, one can solve for the node velocity $v_{i}$,

$v_{i}=\left[\begin{array}{cc}-v_{f D} & F_{r e s, i}<n_{K, i} F_{s K} \\ \frac{n_{K, i} F_{s K}+n_{D, i} F_{s D}-F_{r e s, i}}{n_{K, i} F_{s K} / v_{b K}-n_{D, i} F_{s D} / v_{f D}-\mu} & n_{K, i} F_{s K} \leq F_{r e s, i}<n_{K, i} F_{s K}+n_{D, i} F_{s D} \\ \frac{n_{K, i} F_{s K}+n_{D, i} F_{s D}-F_{r e s, i}}{n_{K, i} F_{s K} / v_{f K}-n_{D, i} F_{s D} / v_{b D}-\mu} & n_{K, i} F_{s K}+n_{D, i} F_{s D} \leq F_{r e s, i} \leq n_{D, i} F_{s D} \\ v_{f K} & n_{D, i} F_{s D}<F_{r e s, i}\end{array}\right.$
Dyneins win

Dyneins win with load

Kinesins win with load

Kinesins win 
the force acting on one kinesin attached to node $i$

$$
F_{K, i}=\left[\begin{array}{cc}
F_{s K}\left(1+v_{f D} / v_{b K}\right) & F_{r e s, i}<n_{K, i} F_{s K} \\
\frac{F_{s K}\left(-n_{D, i} F_{s D}\left(1 / v_{b K}+1 / v_{f D}\right)+F_{r e s, i} / v_{b K}-\mu\right)}{n_{K, i} F_{s K} / v_{b K}-n_{D, i} F_{s D} / v_{f D}-\mu} & n_{K, i} F_{s K} \leq F_{r e s, i}<n_{K, i} F_{s K}+n_{D, i} F_{s D} \\
\frac{F_{s K}\left(-n_{D, i} F_{s D}\left(1 / v_{b D}+1 / v_{f K}\right)+F_{r e s, i} / v_{f K}-\mu\right)}{n_{K, i} F_{s K} / v_{f K}-n_{D, i} F_{s D} / v_{b D}-\mu} & n_{K, i} F_{s K}+n_{D, i} F_{s D} \leq F_{r e s, i} \leq n_{D, i} F_{s D} \\
\frac{-n_{D, i} F_{s D}\left(v_{f K} / v_{b D}+1\right)+F_{e x t, i}-\mu v_{f K}}{n_{K, i}} & n_{D, i} F_{s D}<F_{r e s, i}
\end{array}\right.
$$

and the force acting on one dynein attached to node $i$

$$
F_{D, i}=\left[\begin{array}{cc}
\frac{n_{K, i} F_{s K}\left(v_{f D} / v_{b K}+1\right)-F_{r e s, i}-v_{f D} \mu}{-n_{D, i}} & F_{r e s, i}<n_{K, i} F_{s K} \\
\frac{F_{s D}\left(n_{K, i} F_{s K}\left(1 / v_{b K}+1 / v_{f D}\right)-F_{r e s, i} / v_{f D}-\mu\right)}{n_{K, i} F_{s K} / v_{b K}-n_{D, i} F_{s D} / v_{f D}-\mu} & n_{K, i} F_{s K} \leq F_{r e s, i}<n_{K, i} F_{s K}+n_{D, i} F_{s D} \\
\frac{F_{s D}\left(n_{K, i} F_{s K}\left(1 / v_{b D}+1 / v_{f K}\right)-F_{r e s, i} / v_{b D}-\mu\right)}{n_{K, i} F_{s K} / v_{f K}-n_{D, i} F_{s D} / v_{b D}-\mu} & n_{K, i} F_{s K}+n_{D, i} F_{s D} \leq F_{r e s, i} \leq n_{D, i} F_{s D} \\
F_{s D}\left(1+v_{f K} / v_{b D}\right) & n_{D, i} F_{s D}<F_{r e s, i}
\end{array} .\right.
$$

These equations can be written in more concise notation as

$$
\begin{gathered}
v_{i}=\frac{-\left(a_{K} n_{K, i} F_{s K}+a_{D} n_{D, i} F_{s D}-a_{D} a_{K} F_{r e s, i}\right)}{a_{D} n_{D, i} F_{s D} / v_{0 D}-a_{K} n_{K, i} F_{s K} / v_{0 K}+a_{K} a_{D} \mu} \\
F_{K, i}=\frac{F_{s K}\left(-n_{D, i} F_{s D}\left(1 / v_{0 K}+1 / v_{0 D}\right)+a_{K} F_{r e s, i} / v_{0 K}-a_{K} \mu\right)}{a_{K} n_{K, i} F_{s K} / v_{0 K}-a_{D} n_{D, i} F_{s D} / v_{0 D}-a_{K} a_{D} \mu}
\end{gathered}
$$

and

$$
F_{D, i}=\frac{F_{s D}\left(n_{K, i} F_{s K}\left(1 / v_{0 K}+1 / v_{0 D}\right)-a_{D} F_{r e s, i} / v_{0 D}-a_{D} \mu\right)}{a_{K} n_{K, i} F_{s K} / v_{0 K}-a_{D} n_{D, i} F_{s D} / v_{0 D}-a_{K} a_{D} \mu}
$$

where the parameters are defined as follows

$$
a_{K}=\left[\begin{array}{cl}
0 & F_{\text {res }, i}<n_{K, i} F_{s K} \\
1 & n_{K, i} F_{s K} \leq F_{\text {res }, i}
\end{array} \quad a_{D}=\left[\begin{array}{cc}
0 & n_{D, i} F_{s D}<F_{r e s, i} \\
1 & F_{r e s, i} \leq n_{D, i} F_{s D}
\end{array}\right.\right.
$$

and

$$
\begin{aligned}
& v_{0 K}=\left[\begin{array}{lll}
v_{b K} & F_{r e s, i}<n_{K, i} F_{s K}+n_{D, i} F_{s D} & \text { Dyneins win } \\
v_{f K} & n_{K, i} F_{s K}+n_{D, i} F_{s D} \leq F_{r e s, i} & \text { Kinesins win }
\end{array}\right. \\
& v_{0 D}=\left[\begin{array}{lll}
v_{f D} & F_{r e s, i}<n_{K, i} F_{s K}+n_{D, i} F_{s D} & \text { Dyneins win } \\
v_{b D} & n_{K, i} F_{s K}+n_{D, i} F_{s D} \leq F_{r e s, i} & \text { Kinesins win }
\end{array}\right.
\end{aligned}
$$




\begin{tabular}{|c|c|c|c|c|c|}
\hline $\begin{array}{c}\text { Condition } \\
\text { of } \\
\text { regime } \\
\end{array}$ & $F_{r e s, i}<$ & $F_{r e s, i}<$ & $\begin{array}{c}n_{K, i} F_{s K} \\
+n_{D, i} F_{s D} \\
\end{array}$ & $\leq F_{r e s, i} \leq$ & $<F_{r e s, i}$ \\
\hline Direction & & & & & \\
\hline Speed & Fast & Slow & & Slow & Fast \\
\hline$a_{K}$ & 0 & 1 & & 1 & 1 \\
\hline$a_{D}$ & 1 & 1 & & 1 & 0 \\
\hline$v_{0 K}$ & $v_{b K}$ & $v_{b K}$ & & $v_{f K}$ & $v_{f K}$ \\
\hline$v_{0 D}$ & $v_{f D}$ & $v_{f D}$ & & $v_{b D}$ & $v_{b D}$ \\
\hline
\end{tabular}

Table 2: Conditions of regime of motion of node $i$, direction, speed and parameter values $v_{0 *}$ and $a_{*}$. Values for parameters can be found in Table 3

Values of $v_{0 *}$ and $a_{*}$ are also given in Table 2. The dynamics of the motor molecules are the same as [13] except for the dissociation rate for dynein where we use the form given in [14. The effective binding rate is

$$
\left(N_{*}-n_{*}\right) \pi_{*}
$$

where $*$ is either $D$ or $K$ for dynein or kinesin. The effective off rate varies between two functions for each of the motors. There is an exponential function which is commonly used in modeling molecular transport and a catch bond version for each motor based on the experimental form reported in [14]. For kinesin, the two functional forms are given by:

Exponential

$$
n_{K} \epsilon_{K} \exp \left[\frac{\left|F_{K}\right|}{F_{d K}}\right]
$$

\section{Catch Bond}

$$
\left\{\begin{array}{ll}
\left.n_{K} \epsilon_{K}\left(2+0.689\left|\frac{F_{K}}{F_{d K}}\right|\right)\right) & F_{K}<F_{s K} \\
n_{K} \epsilon_{K} \exp \left[\left|\frac{F_{K}}{F_{d K}}\right|\right] & F_{K} \geq F_{s K}
\end{array} .\right.
$$

For dynein, the functions are:

Exponential

$$
n_{D} \epsilon_{D} \exp \left[\frac{\left|F_{D}\right|}{F_{d D}}\right]
$$

Catch Bond

$$
\begin{cases}\frac{n_{D} \epsilon_{D} \exp \left[\frac{F_{s D}}{F_{d D}}\right]\left(1-b \exp \left(\frac{-F_{s D}}{F_{d D}}\right)\right)}{\left(1-b \exp \left(\frac{-F_{D}}{F_{d D}}\right)\right)} & F_{D}>F_{s D} \\ n_{D} \epsilon_{D} \exp \left[\frac{\left|F_{D}\right|}{F_{d D}}\right] & F_{D} \leq F_{s D}\end{cases}
$$

where $b=0.602 \exp \left(\frac{F_{s D}}{F_{d D}}\right)$. The force in these equations is given in $\mathrm{pN}$. Since we 
do not use the same parameters as 14] we used functional forms which give the same qualitative features as the experimental dissociation rates given there. For example, the discontinuity in the kinesin dissociation rate occurs at the value of $F_{d K}$ and the jump in the discontinuity is about 38 percent of the maximum value.

\subsection{Parameters}

The drag coefficient for a node is given by $\mu=\frac{3 \pi \eta L}{\ln (\rho)+v}$ using equation 2 in 58 for the translational diffusion coefficient of a rod, Einsteins relation, and assuming a low Reynold's number. The length of the rod is $L$, the dynamic viscosity of the fluid is $\eta, \rho=L / d$ where $d$ is the diameter of the rod. Letting $L=\ell=0.5 \mu \mathrm{m}$ the rest length of the springs between the nodes, and $d=0.01$ $\mu \mathrm{m}$, we find $\rho=50$. and $v=0.3233$. Thus $\mu=1.1127 \times 10^{-6} \eta$ in metric units. Determining $\eta$ the viscosity of the cytoplasm is somewhat problematic. Although the cytosol is mainly water, if it was considered a homogeneous fluid for the motion of an intermediate filament of $0.5 \mu \mathrm{m}$ in length one would assume it would have a much larger viscosity due to the cytoskeletal structure which would impede the motion of the short intermediate filament. Two types of measurements of the viscosity of the cytoplasm give very different values. When estimating the viscosity by observing a small molecule diffuse in the cytosol the range is $0.8-44 \mathrm{mPa} \mathrm{s}$ 59. Yet when estimating the viscosity by fitting force relaxation curves to the cytoplasm the range is 50-166 $\mathrm{Pa} \mathrm{s}$ 60, 61. We choose a value in between the two ranges and assume $\eta=1.08 \mathrm{~Pa} \mathrm{~s}$, about 1000 times higher than water. This gives $\mu=1.2026 \times 10^{-12}$ in $\mathrm{Ns} / \mu \mathrm{m}$.

Young's modulus is defined to be $\mathcal{Y}=\frac{\sigma}{\varepsilon}$, where $\sigma=F / A$ is the stress and the strain is $\varepsilon=\Delta L / L$. Using Hooke's law, $F=\alpha \Delta L$, we get a relationship between the spring constant $\alpha$ and the Young's modulus given by $\alpha=\frac{\mathcal{Y} A}{L}$. The 575 elastic modulus of vimentin has been estimated to be 8-9 MPa 31], $900 \mathrm{MPa}$ by [32, and 6.5 MPa for another keratin-like based filament 33. For a 10 percent change of length with forces on the order of $0.18 \mathrm{pN}$ a Young's modulus of 22 $\mathrm{MPa}$ is needed. This lies in the range of experimentally measured values and compares well with values used in 34. Thus the base case for our simulations uses a spring constant, $\alpha=0.345 \times 10^{-8} \mathrm{~N} / \mu \mathrm{m}$, which corresponds to a Young's modulus of $22 \mathrm{MPa}$. We also run simulations with more compliant filaments and less compliant filaments by multiplying the spring constant by some factor from $10^{-3}$ to $10^{3}$.

The parameters used for the motor molecules properties are given in Table 3 .

\subsection{Numerical Methods Used for Simulations}

The system of differential equations is solved using CVODE 62]. The binding and unbinding events are determined using a modified Gillespie algorithm [63] which assumes that the binding events on each node are independent of

590 all the other nodes. This assumption is for computational convenience and is not true since the external force on a node depends on the number of molecules 
Table 3: Parameters used for simulations

\begin{tabular}{||l||l||l||}
\multicolumn{2}{c}{ Kinesin } & Dynein \\
\hline$v_{f} \mu m / \mathrm{s}$ & 1.0 & 1.0 \\
\hline$v_{b} \mu \mathrm{m} / \mathrm{s}$ & 0.006 & 0.006 \\
\hline$F_{d} \mathrm{pN}$ & 3 & 3 \\
\hline$F_{s} \mathrm{pN}$ & -6 & 7 \\
\hline$\epsilon \mathrm{s}^{-1}$ & 1 & 0.25 \\
\hline$\pi \mathrm{s}^{-1}$ & 5 & 1.5 \\
\hline \hline
\end{tabular}

bound to the other nodes. The modification to the algorithm is the binding or unbinding of motor molecules are constrained to occur at times which are integer multiples of $\Delta t=0.01$ seconds. Although the Gillespie algorithm does not require the time constrain assumption, we use it to speed up the simulations. The effects of this assumption are to artificially lengthen the time a motor remains attached to a filament and to lengthen the time a motor molecule must wait before attaching to a filament. Numerical simulations varying the size of $\Delta t$ did not show qualitatively different solutions provided $\Delta t$ was small enough.

\section{Acknowledgments}

The authors would like to thank the Isaac Newton Institute for Mathematical Sciences for support and hospitality during the programme "Coupling Geometric PDE's with Physics for Cell Morphology, Motility and Pattern Formation" when the work on this paper was undertaken. This work was supported by: EPSRC ${ }_{605}$ grant numbers EP/K032208/1 and EP/R014604/1 and by La Ligue contre le cancer and the Institut Pasteur. 


\section{References}

[1] C. Leduc, S. Etienne-Manneville, Intermediate filaments in cell migration and invasion: the unusual suspects, Current opinion in cell biology 32 (2015) 102-112.

[2] C. Leduc, S. Etienne-Manneville, Regulation of microtubule-associated motors drives intermediate filament network polarization, J Cell Biol 216 (6) (2017) 1689-1703.

[3] R. E. Leube, M. Moch, R. Windoffer, Intracellular motility of intermediate filaments, Cold Spring Harbor Perspectives in Biology 9 (6) (2017) a021980.

[4] J. D. Fenn, C. M. Johnson, J. Peng, P. Jung, A. Brown, Kymograph analysis with high temporal resolution reveals new features of neurofilament transport kinetics, Cytoskeleton.

[5] A. Robert, C. Hookway, V. I. Gelfand, Intermediate filament dynamics: What we can see now and why it matters, BioEssays.

[6] C. Hookway, L. Ding, M. W. Davidson, J. Z. Rappoport, G. Danuser, V. I. Gelfand, Microtubule-dependent transport and dynamics of vimentin intermediate filaments, Molecular biology of the cell 26 (9) (2015) 16751686.

[7] B. T. Helfand, L. Chang, R. D. Goldman, Intermediate filaments are dynamic and motile elements of cellular architecture, Journal of cell science 117 (2) (2004) 133-141.

[8] R. D. Vale, T. S. Reese, M. P. Sheetz, Identification of a novel forcegenerating protein, kinesin, involved in microtubule-based motility, Cell 42 (1) (1985) 39-50.

[9] S. T. Brady, A novel brain atpase with properties expected for the fast axonal transport motor, Nature 317 (6032) (1985) 73-75.

[10] B. M. Paschal, H. S. Shpetner, R. B. Vallee, Map 1c is a microtubuleactivated atpase which translocates microtubules in vitro and has dyneinlike properties., The Journal of cell biology 105 (3) (1987) 1273-1282.

[11] R. Lye, M. Porter, J. Scholey, J. McIntosh, Identification of a microtubulebased cytoplasmic motor in the nematode c. elegans, Cell 51 (2) (1987) 309-318.

[12] W. O. Hancock, Bidirectional cargo transport: moving beyond tug of war, Nature reviews Molecular cell biology 15 (9) (2014) 615.

[13] M. J. Müller, S. Klumpp, R. Lipowsky, Tug-of-war as a cooperative mechanism for bidirectional cargo transport by molecular motors, Proceedings of the National Academy of Sciences 105 (12) (2008) 4609-4614. 
[14] A. Kunwar, S. K. Tripathy, J. Xu, M. K. Mattson, P. Anand, R. Sigua, M. Vershinin, R. J. McKenney, C. Y. Clare, A. Mogilner, et al., Mechanical stochastic tug-of-war models cannot explain bidirectional lipid-droplet transport, Proceedings of the National Academy of Sciences 108 (47) (2011) 18960-18965.

[15] A. Takshak, A. Kunwar, Importance of anisotropy in detachment rates for force production and cargo transport by a team of motor proteins, Protein Science 25 (5) (2016) 1075-1079.

[16] C. Leidel, R. A. Longoria, F. M. Gutierrez, G. T. Shubeita, Measuring molecular motor forces in vivo: implications for tug-of-war models of bidirectional transport, Biophys J 103 (3) (2012) 492-500. doi:

[17] S. K. Vogel, N. Pavin, N. Maghelli, F. Jülicher, I. M. Tolić-Nørrelykke, Self-organization of dynein motors generates meiotic nuclear oscillations, PLoS biology 7 (4) (2009) e1000087.

[18] G. Craciun, A. Brown, A. Friedman, A dynamical system model of neurofilament transport in axons, Journal of Theoretical Biology 237 (3) (2005) 316 -322. doi:https://doi.org/10.1016/j.jtbi.2005.04.018.

URL http://www.sciencedirect.com/science/article/pii/ S0022519305001827

[19] R. H. Lee, C. S. Mitchell, Axonal transport cargo motor count versus 665 average transport velocity: Is fast versus slow transport really single versus multiple motor transport?, Journal of Theoretical Biology 370 (Supplement C) (2015) 39-44. doi:https://doi.org/10.1016/j.jtbi.2015.01.010.

1. URL http://www.sciencedirect.com/science/article/pii/ S0022519315000193

670 [20] C. Xue, B. Shtylla, A. Brown, A stochastic multiscale model that explains the segregation of axonal microtubules and neurofilaments in neurological diseases, PLoS computational biology 11 (8) (2015) e1004406.

[21] I. Kuznetsov, A. Kuznetsov, Analytical comparison between nixon-

1. logvinenko's and jung-brown's theories of slow neurofilament trans-

675 port in axons, Mathematical Biosciences 245 (2) (2013) 331 - 339. doi:https://doi.org/10.1016/j.mbs.2013.08.001.

a URL http://www.sciencedirect.com/science/article/pii/ S0025556413001867

[22] S. Portet, A. Madzvamuse, A. Chung, R. E. Leube, R. Windoffer, Keratin dynamics: modeling the interplay between turnover and transport, PloS one 10 (3) (2015) e0121090.

[23] J. R. Kardon, S. L. Reck-Peterson, R. D. Vale, Regulation of the processivity and intracellular localization of saccharomyces cerevisiae dynein 
n by dynactin, Proc Natl Acad Sci U S A 106 (14) (2009) 5669-74. doi: 10.1073/pnas.0900976106.

[24] J.-B. Manneville, M. Jehanno, S. Etienne-Manneville, Dlg1 binds gkap to control dynein association with microtubules, centrosome positioning, and cell polarity, J Cell Biol 191 (3) (2010) 585-98. doi:10.1083/jcb. 201002151 .

[25] D. Pathak, S. Thakur, R. Mallik, Fluorescence microscopy applied to intracellular transport by microtubule motors, J Biosci 43 (3) (2018) 437-445.

[26] H. Herrmann, U. Aebi, Intermediate filaments: molecular structure, assembly mechanism, and integration into functionally distinct intracellular scaffolds, Annu Rev Biochem 73 (2004) 749-89. doi:10.1146/annurev. biochem.73.011303.073823.

[27] S. Köster, D. A. Weitz, R. D. Goldman, U. Aebi, H. Herrmann, Intermediate filament mechanics in vitro and in the cell: from coiled coils to filaments, fibers and networks, Curr Opin Cell Biol 32 (2015) 82-91. doi:10.1016/j.ceb.2015.01.001.

[28] T. L. Blasius, N. Reed, B. M. Slepchenko, K. J. Verhey, Recycling of kinesin-1 motors by diffusion after transport, PLoS One 8 (9) (2013) e76081. doi:10.1371/journal.pone.0076081.

[29] A. K. Rai, A. Rai, A. J. Ramaiya, R. Jha, R. Mallik, Molecular adaptations allow dynein to generate large collective forces inside cells, Cell 152 (1-2) (2013) 172-82. doi:10.1016/j.cell.2012.11.044

[30] M. P. Nicholas, F. Berger, L. Rao, S. Brenner, C. Cho, A. Gennerich, Cytoplasmic dynein regulates its attachment to microtubules via nucleotide state-switched mechanosensing at multiple aaa domains, Proc Natl Acad Sci U S A 112 (20) (2015) 6371-6. doi:10.1073/pnas.1417422112.

[31] Z. Qin, C.-C. Chou, L. Kreplak, M. Buehler, Structural, mechanical and functional properties of intermediate filaments from the atomistic to the cellular scales, in: S. Li, B. Sun (Eds.), Advances in Cell Mechanics, Springer, 2011, Ch. 4, pp. 139-140.

[32] C. Guzman, S. Jeney, L. Kreplak, S. Kasas, A. Kulik, U. Aebi, L. Forro, Exploring the mechanical properties of single vimentin intermediate filaments by atomic force microscopy, Journal of molecular biology 360 (3) (2006) 623-630.

[33] L. Kreplak, D. Fudge, Biomechanical properties of intermediate filaments: from tissues to single filaments and back, Bioessays 29 (1) (2007) 26-35.

${ }_{720}$ [34] J. Block, H. Witt, A. Candelli, E. J. Peterman, G. J. Wuite, A. Janshoff, S. Köster, Nonlinear loading-rate-dependent force response of individual vimentin intermediate filaments to applied strain, Physical Review Letters 118 (4) (2017) 048101. 
[35] M. Vershinin, B. C. Carter, D. S. Razafsky, S. J. King, S. P. Gross, Multiplemotor based transport and its regulation by tau, Proc Natl Acad Sci U S A 104 (1) (2007) 87-92. doi:10.1073/pnas.0607919104.

[36] G. T. Shubeita, S. L. Tran, J. Xu, M. Vershinin, S. Cermelli, S. L. Cotton, M. A. Welte, S. P. Gross, Consequences of motor copy number on the intracellular transport of kinesin-1-driven lipid droplets, Cell 135 (6) (2008) 1098-107. doi:10.1016/j.cell.2008.10.021.

[37] K. S. Thorn, J. A. Ubersax, R. D. Vale, Engineering the processive run length of the kinesin motor, J Cell Biol 151 (5) (2000) 1093-100.

[38] L. Chang, R. D. Goldman, Intermediate filaments mediate cytoskeletal crosstalk, Nat Rev Mol Cell Biol 5 (8) (2004) 601-13. doi:10.1038/ nrm1438

[39] S. Klein, C. Appert-Rolland, L. Santen, Motility states in bidirectional cargo transport, EPL (Europhysics Letters) 111 (6) (2015) 68005.

[40] G. Arpă̆, S. Shastry, W. O. Hancock, E. Tüzel, Transport by populations of fast and slow kinesins uncovers novel family-dependent motor characteristics important for in vivo function, Biophysical journal 107 (8) (2014) 1896-1904.

[41] J. O. L. Andreasson, B. Milic, G.-Y. Chen, N. R. Guydosh, W. O. Hancock, S. M. Block, Examining kinesin processivity within a general gating framework, Elife 4. doi:10.7554/eLife.07403.

[42] S.-K. Guo, X.-X. Shi, P.-Y. Wang, P. Xie, Processivity of dimeric kinesin-1 molecular motors, FEBS Open Bio 8 (8) (2018) 1332-1351. doi:10.1002/ 2211-5463.12486.

[43] M. Sawant, R. Leube, Consequences of keratin phosphorylation for cytoskeletal organization and epithelial functions, in: International review of cell and molecular biology, Vol. 330, Elsevier, 2017, pp. 171-225.

[44] N. T. Snider, M. B. Omary, Post-translational modifications of intermediate filament proteins: mechanisms and functions, Nature reviews Molecular cell biology 15 (3) (2014) 163.

[45] A. Uchida, G. Çolakoğlu, L. Wang, P. C. Monsma, A. Brown, Severing and end-to-end annealing of neurofilaments in neurons, Proceedings of the National Academy of Sciences 110 (29) (2013) E2696-E2705.

[46] M. A. Cianfrocco, M. E. DeSantis, A. E. Leschziner, S. L. Reck-Peterson, Mechanism and regulation of cytoplasmic dynein, Annual review of cell and developmental biology 31 (2015) 83-108.

[47] M. P. Nicholas, P. Höök, S. Brenner, C. L. Wynne, R. B. Vallee, A. Gennerich, Control of cytoplasmic dynein force production and processivity by its c-terminal domain, Nature communications 6. 
[48] J. Howard, et al., Mechanics of motor proteins and the cytoskeleton, Sinauer associates Sunderland, MA, 2001.

[49] L. Wang, A. Brown, Rapid movement of microtubules in axons, Current Biology 12 (17) (2002) 1496-1501.

[50] A. L. Jolly, H. Kim, D. Srinivasan, M. Lakonishok, A. G. Larson, V. I. Gelfand, Kinesin-1 heavy chain mediates microtubule sliding to drive changes in cell shape, Proc Natl Acad Sci U S A 107 (27) (2010) 12151-6. doi:10.1073/pnas.1004736107.

[51] S. Sivaramakrishnan, J. V. DeGiulio, L. Lorand, R. D. Goldman, K. M. Ridge, Micromechanical properties of keratin intermediate filament networks, Proceedings of the National Academy of Sciences 105 (3) (2008) 889-894.

[52] S. Portet, Dynamics of in vitro intermediate filament length distributions, Journal of theoretical biology 332 (2013) 20-29.

[53] P. Steinert, L. Marekov, D. Parry, Diversity of intermediate filament structure. evidence that the alignment of coiled-coil molecules in vimentin is different from that in keratin intermediate filaments., Journal of Biological Chemistry 268 (33) (1993) 24916-24925.

[54] N. Mücke, L. Kreplak, R. Kirmse, T. Wedig, H. Herrmann, U. Aebi, J. Langowski, Assessing the flexibility of intermediate filaments by atomic force microscopy, J Mol Biol 335 (5) (2004) 1241-50.

[55] M. Schopferer, H. Bär, B. Hochstein, S. Sharma, N. Mücke, H. Herrmann, N. Willenbacher, Desmin and vimentin intermediate filament networks: their viscoelastic properties investigated by mechanical rheometry, Journal of molecular biology 388 (1) (2009) 133-143.

[56] B. T. Helfand, A. Mikami, R. B. Vallee, R. D. Goldman, A requirement for cytoplasmic dynein and dynactin in intermediate filament network assembly and organization, J Cell Biol 157 (5) (2002) 795-806. doi:10.1083/jcb.200202027.

[57] V. Prahlad, M. Yoon, R. D. Moir, R. D. Vale, R. D. Goldman, Rapid movements of vimentin on microtubule tracks: kinesin-dependent assembly of intermediate filament networks, J Cell Biol 143 (1) (1998) 159-70.

[58] J. G. De La Torre, C. LOPEZ, Dimensions of short, rodlike macromolecules from, Biopolymers 23 (1984) 611-615.

[59] T. Kalwarczyk, N. Ziebacz, A. Bielejewska, E. Zaboklicka, K. Koynov, J. Szymanski, A. Wilk, A. Patkowski, J. Gapinski, H.-J. r. Butt, et al., Comparative analysis of viscosity of complex liquids and cytoplasm of mammalian cells at the nanoscale, Nano letters 11 (5) (2011) 2157-2163. 
[60] E. Moeendarbary, L. Valon, M. Fritzsche, A. R. Harris, D. A. Moulding, A. J. Thrasher, E. Stride, L. Mahadevan, G. T. Charras, The cytoplasm of living cells behaves as a poroelastic material, Nature materials 12 (3) (2013) 253-261.

[61] E. Sackmann, F. Keber, D. Heinrich, J. S. Langer, Physics of cellular movements, Annu. Rev. Condens. Matter Phys. 1 (2010) 257-276. doi:10.1146/annurev-conmatphys-070909-104105.

[62] A. C. Hindmarsh, P. N. Brown, K. E. Grant, S. L. Lee, R. Serban, D. E. Shumaker, C. S. Woodward, Sundials: Suite of nonlinear and differential/algebraic equation solvers, ACM Transactions on Mathematical Software (TOMS) 31 (3) (2005) 363-396.

[63] D. T. Gillespie, Stochastic simulation of chemical kinetics, Annu. Rev. Phys. Chem. 58 (2007) 35-55.

\section{Supplemental Data}

Simulation results are given for the all combinations of off rates and all combinations of initial conditions.

\subsection{Off Rates - Dynein Catch Bond, Kinesin Catch Bond}

In this section all simulations have the catch bond off rate for both motor molecules.

There does not appear to be a trend in the velocity as the filament length increases. It is clear that for low numbers of motor molecules the standard deviation for the velocity is greater. For filament with a low spring constant the average velocity is non-negative but individual filaments have positive and negative velocities. For normal filament elasticity the filament velocity is non-

825 positive and again individual filaments move in both directions. For the filaments with the highest spring constant the average velocity is closer to zero and takes both positive and negative values for short filaments. For longer filaments the velocity is close to zero. For filaments with the maximum motor molecules 16 or 32 the velocity is almost zero. Although the initial conditions do affect slightly the velocity the trends described above are independent of the initial conditions (see figures 15,16 , and 17).

Since the filament speed is an average of an averaged, one would expect the standard deviations to be smaller than those for the velocity and they are. For all simulations the filament speed decreases as the maximum number of 835 motor molecules is increased. The initial conditions do not seem to affect the speed (see figure 16). For low filament stiffness one can see a slight increase in the speed of the filament as the filament length increases. For normal filament elasticity there is a decrease and then increase in speed as the filament length increases. Finally for very stiff filaments the speed decreases quickly with increasing filament length. The filament speeds in the simulations are the same 
order of magnitude with experimental values although they are a little low. It is unclear whether speeds measured experimentally are in a transient phase of the dynamics or near a steady state.

The last row of figure 6 shows the location of the first node on filaments as a function of time. Several realizations are shown for each parameter set. Compare these panels with the panels on the first row of figure 6. Notice the spatial scale for panel 3(c) is different from panels 3(a) and 3(b). In panels 3 it is clear, particularly for filaments with low spring constants (plotted in red), that although filaments, in these cases, generally move in the positive direction there ${ }_{850}$ are brief periods where they move in the negative direction. Filaments with the lowest spring constant on average have a positive velocity for all parameter values shown, filaments with normal elasticity (plotted in green) have almost no velocity, negative, and positive depending on the parameters, and filaments with a high spring constant (plotted in blue) have a low average velocity which is negative. In panel 3(a) the filaments with high spring contant have a transient behavior until somewhere near time 800 (compare with figure 14 panel 2(c)).

Figure 14 panels 1 show the normalized number of motor molecules at the end of the simulations, the motor molecule with the greatest number on the filament, and whether the filament is approaching a steady state with regards 860 to the number of motor molecules attached. In panels 1, the maximum number of motor molecules varies with the rows and the filament length varies with the columns. Most simulations seem to be close to a steady state. Additionally when the criterion is changed from less than a 1 percent change to less than five percent change, all the simulations for this figure meet the condition. Due to the parameter set it is possible that the velocity of a filaments with more kinesin is dominated by the dynein and moves in the direction dictated by dynein. By comparing the position of filaments with normal elasticity (the green lines) in figure 6 panel 3(b) with the appropriate square in figure 14 panel 1(b) and panel 2(b) one can see that there is more kinesin on the filament but the filament is 870 moving in a direction dictated by dynein. The same is true of the position of the very stiff filaments in panel 3(c) of figure 6 (although the velocity is much smaller). Overall the initial conditions do not seem to play a large role and more kinesin motors are attached to most simulations (see figure 17). Yet for a few simulations the filaments have negative velocity.

In figure 14 panels 2 the normalized number of dynein and kinesin for each filament node as it changes over time are shown. In panels 3,16 realizations are shown with the average over all realizations plotted on the top with double the width. Panels 2(a) and 3(a) show results for simulations with filament length of 5 nodes, $N_{D}=N_{K}=32$, and low filament stiffness. Clearly the kinesin is 80 greater than the dynein and the simulations seems to be fluctuating about a steady state value. Compare this result with the appropriate square in panel 1(a). Panels 2(b) and 3(b) show results for simulations with filament length 10 nodes, $N_{D}=N_{K}=2$, and normal filament elasticity. Again the filaments seem to be near an equilibrium solution. It is unclear which motor molecule ${ }_{885}$ has a higher number on average but panel 2(a) makes it clear that kinesin is higher. Figure 6 shows that the average instantaneous velocity at the end of 

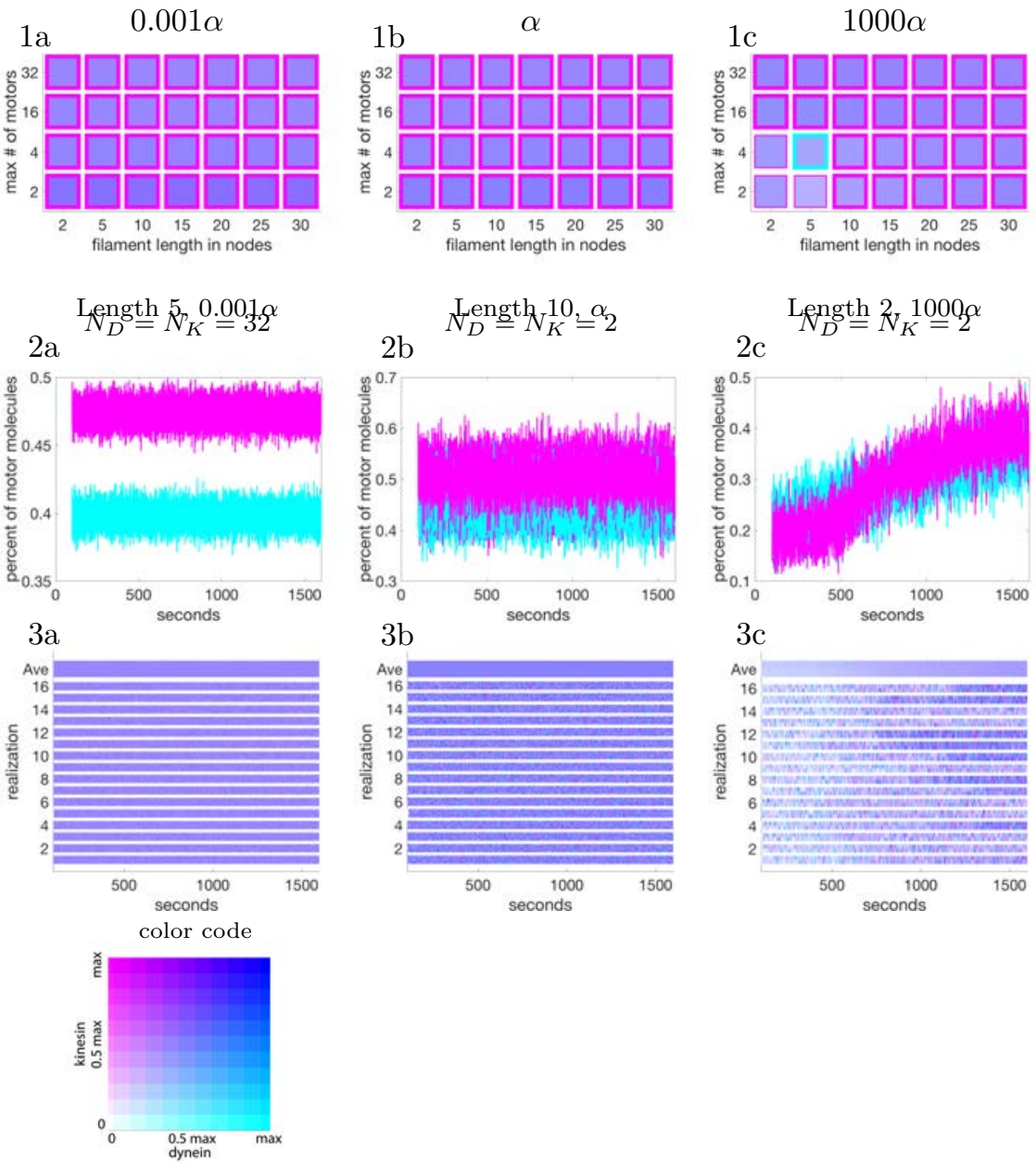

Figure 14: Average number of motor molecules for simulations with both off rates catch bond. Panels 1 show the final number of dynein and kinesin averaged over realizations and nodes. The border color indicates which motor molecule is more abundant. If the border is thick it indicates the number of motor molecules is near a steady state value. If the border is thin, the number of motor molecules is still changing. Panel 1(a) has filament elasticity $0.001 \alpha, 1(\mathrm{~b}) \alpha$ and 1 (c) $1000 \alpha$. Cyan depicts dynein only, magenta kinesin only, and blue means equal amounts of each motor molecule. The graphs 2 and 3 show the number of dynein and kinesin molecules as they change with time. In panels 2 the number of dynein (cyan) and kinesin (magenta) normalized and averaged over each realization is plotted for each node on the filament. Panels 2(a) and 3(a) show simulations for filament length of 5 nodes, $N_{D}=N_{K}=32$, and low filament stiffness. Panel 3(a) shows 16 realizations color coded and the average of all the realizations. The average is plotted twice as wide as each realization. The nodes are plotted on top of each other thus the first node is at the bottom of the color strip and the last node is at the top of the color strip. Panels 2(b) and 3(b) show plots for the same simulations with filament length of 10 nodes, $N_{D}=N_{K}=2$, and normal filament elasticity. Panels 2(c) and 3(c) show plots for simulations with filament length of 2 nodes, $N_{D}=N_{K}=2$, and high filament stiffness. The color key is in the bottom left corner. 
the simulation is negative but some realizations have positive velocity and some have negative velocity, thus on average dynein is driving the motion. Panels 2(c) and 3(c) of figure 14 show plots for simulations with filament length 2 nodes, ${ }_{890} N_{D}=N_{K}=2$, and high filament stiffness. It is clear that the filaments in these simulations are not yet near an equilibrium solution. These plots are typical for all four sets of initial conditions with catch bond off rates (see figure 15, 16 . and 17). Panels 2(a) and 3(a) represent the most commonly seen features of the data and panels 2(c) and 3(c) the least common. Only simulations with high

895 filament stiffness and low maximum number of motor molecules look similar to the results in panels 2(c) and 3(c). This is consistent with the narrow borders seen in panels 1 . The fact that with a higher criterion of 5 percent the narrow borders disappear suggests that panel 2(c) and the other narrow border cases seem to be leveling off to a steady state value. For each set of parameters and 900 initial conditions the expected velocity of the filament is shown in figure 15 The filament elasticity varies with the columns and the initial conditions vary with the rows. 

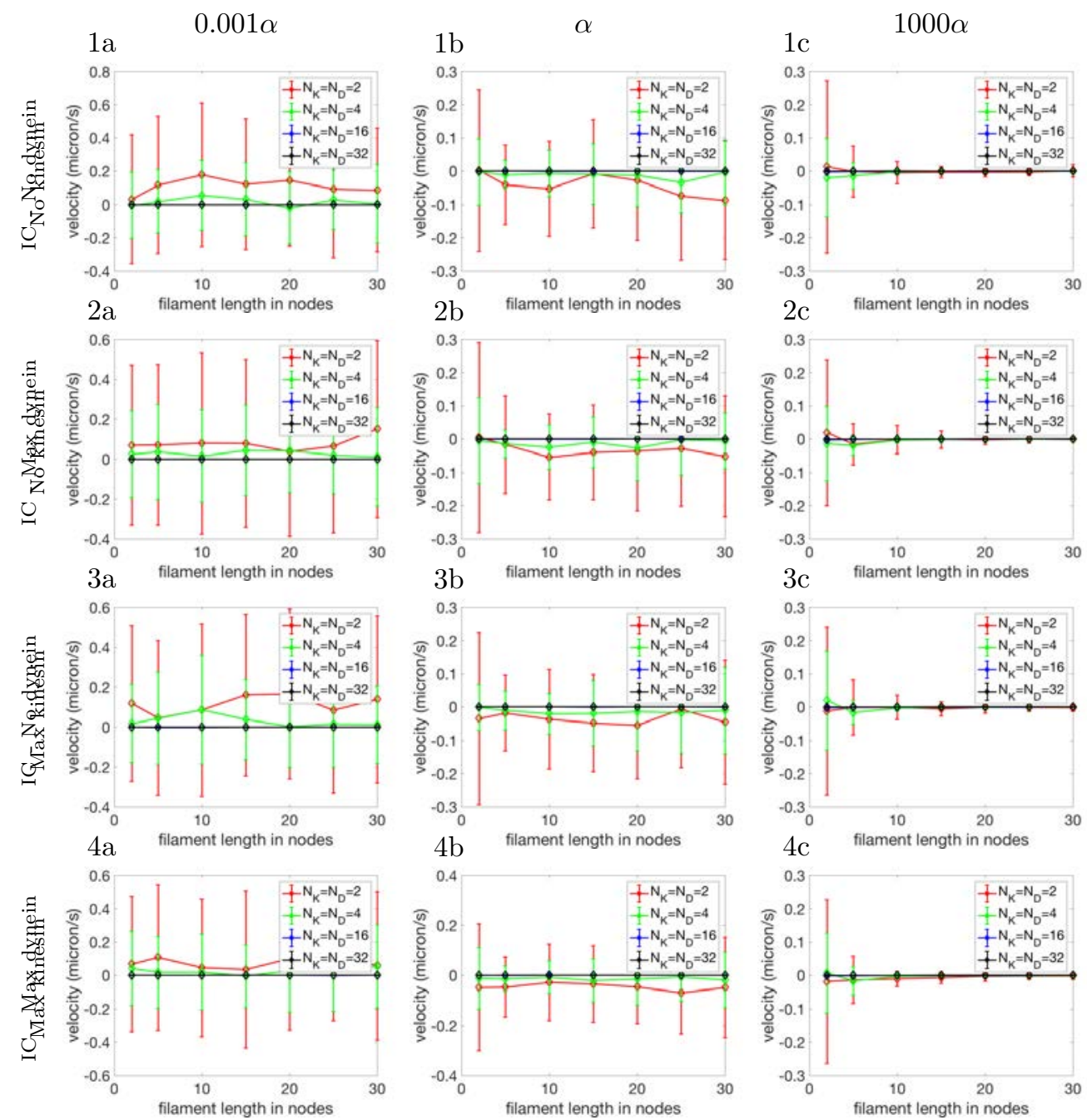

Figure 15: The expected velocity of a filament is plotted as a function of filament length for simulations with both off rates catch bond. The error bars indicate the standard deviation. The initial conditions change for each row and the filament elasticity varies from low to high with the columns. The different plots within each frame are for constant $N_{D}=N_{K}$ with the values given in the legend. In the first row filament node starts with no motor molecules attached, in the second the maximum amount of dynein is attached and no kinesin, in the third row no dynein and the maximum amount of kinesin, and in the fourth row the maximum amount of both motor molecules is attached. In the first column the elasticity of the filament is $0.001 \alpha$, in the second $\alpha$, and in the third $1000 \alpha$. 

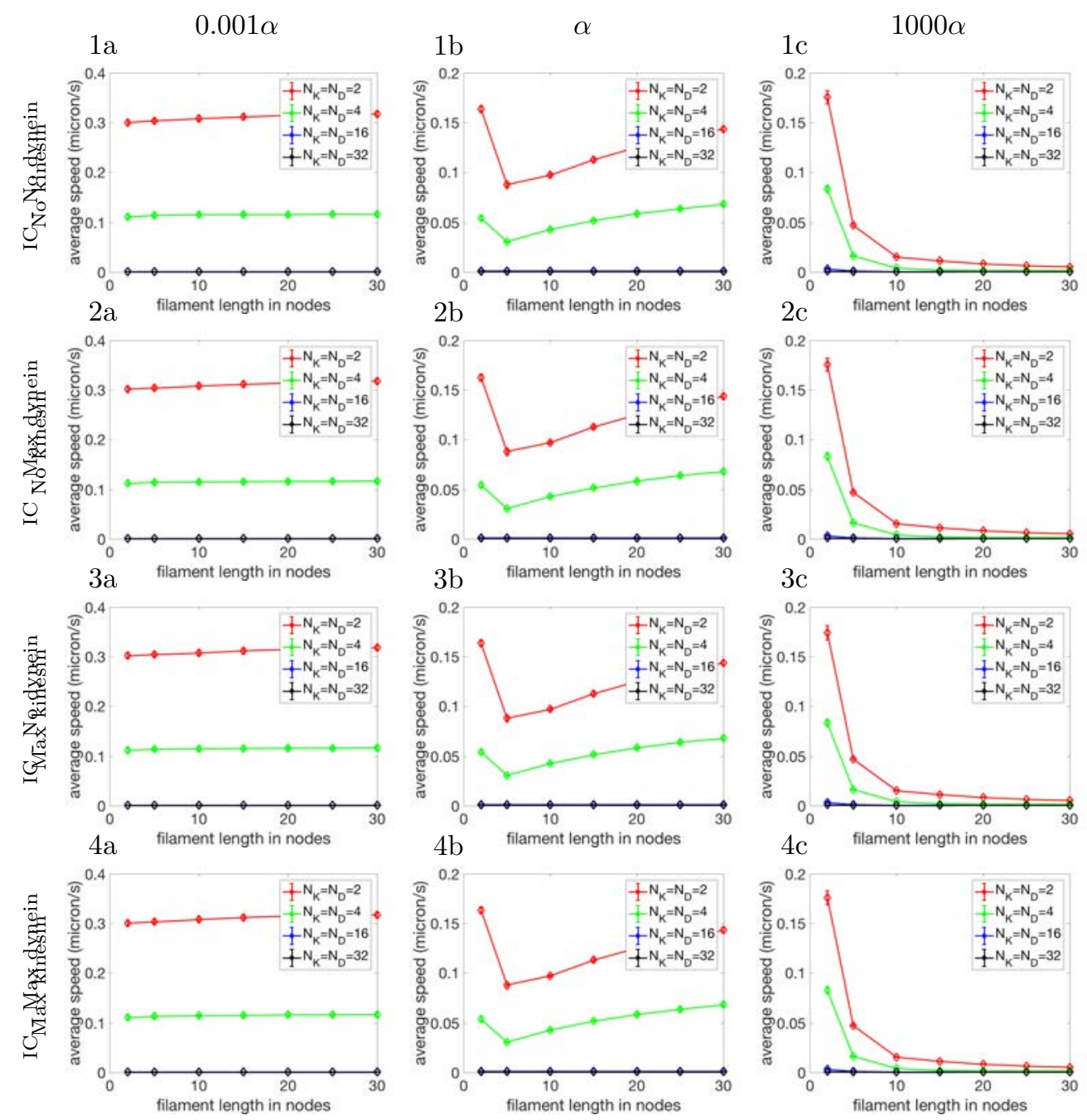

Figure 16: The expected speed of a filament is plotted as a function of filament length for simulations with both off rates catch bond. The error bars indicate the standard deviation. The initial conditions change for each row and the filament elasticity varies from low to high with the columns as in figure 15 The different plots within each frame are for constant $N_{D}=N_{K}$ with the values given in the legend. 

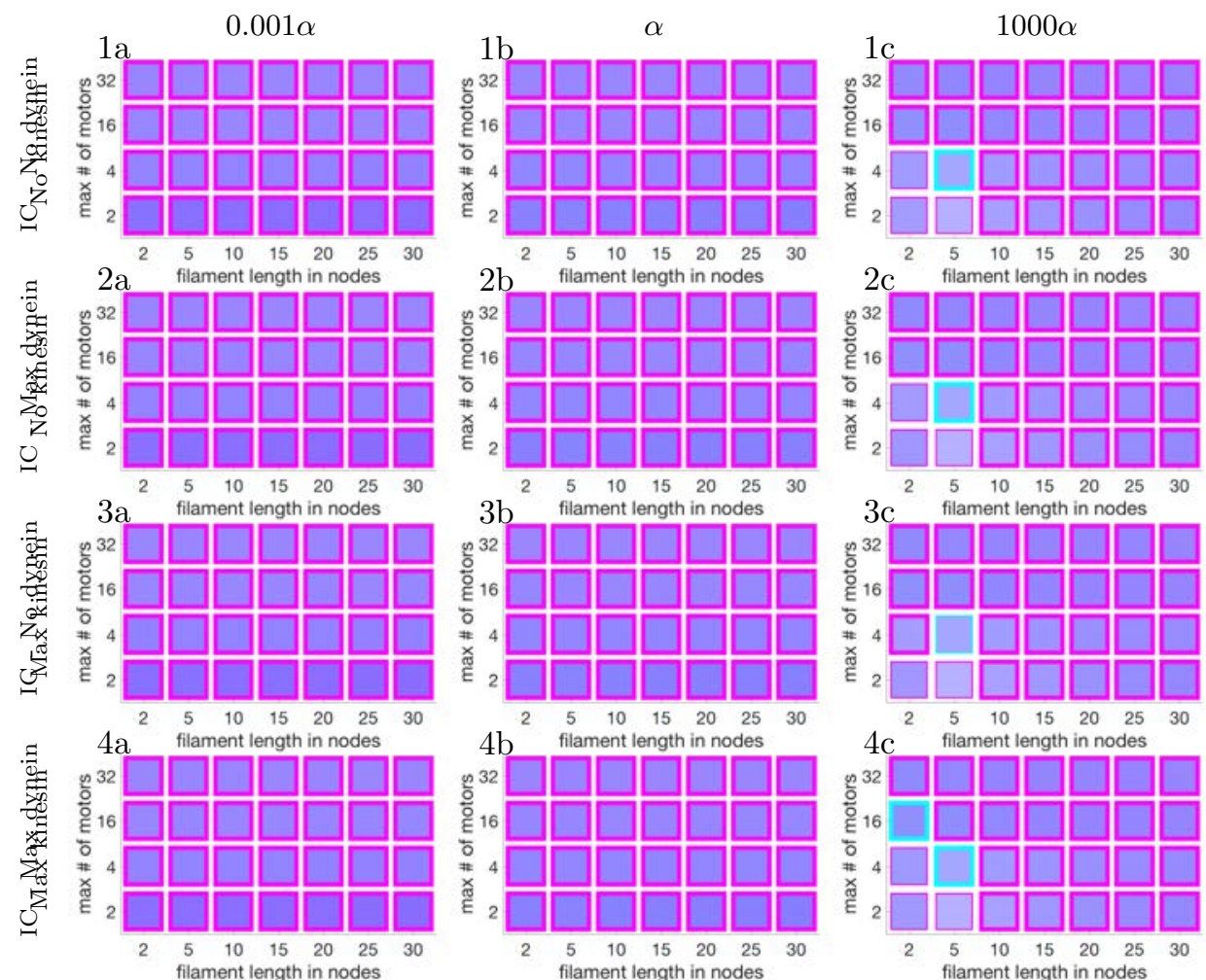

color code

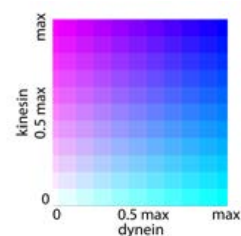

Figure 17: The final number of dynein and kinesin averaged over realizations and nodes for simulations with both off rates catch bond. The border color indicates which motor molecule is more abundant. If the border is thick it indicates the number of motor molecules is near a steady state value. If the border is thin the number of motor molecules is still changing. The initial conditions change for each row and the filament elasticity varies from low to high with the columns as in figure 15. Cyan depicts dynein only, magenta kinesin only, and blue means equal amounts of each motor molecule. The color key is at the bottom left. The time shown is 1600 seconds. 


\subsection{Off Rates - Dynein Catch Bond, Kinesin Exponential}

In this section all the simulations have the catch bond off rate for dynein filament length is 15 nodes. Panels 2 show results for $N_{D}=N_{K}=2$ and panels 3 for $N_{D}=N_{K}=32$. Panels 2(a) and 3(a) have elastic constant $\alpha$ and 2 (b), 3(b), 2(c), and 3(c) have $1000 \alpha$. In the first two columns, the cyan curves are the average value of the dynein for each node averaged over all realizations 945 and the magenta curves are the average value of the kinesin value for each node. There are 15 cyan and magenta curve, as determined by the length of the filament although in the graphs they are hard to distinguish. As is expected, 

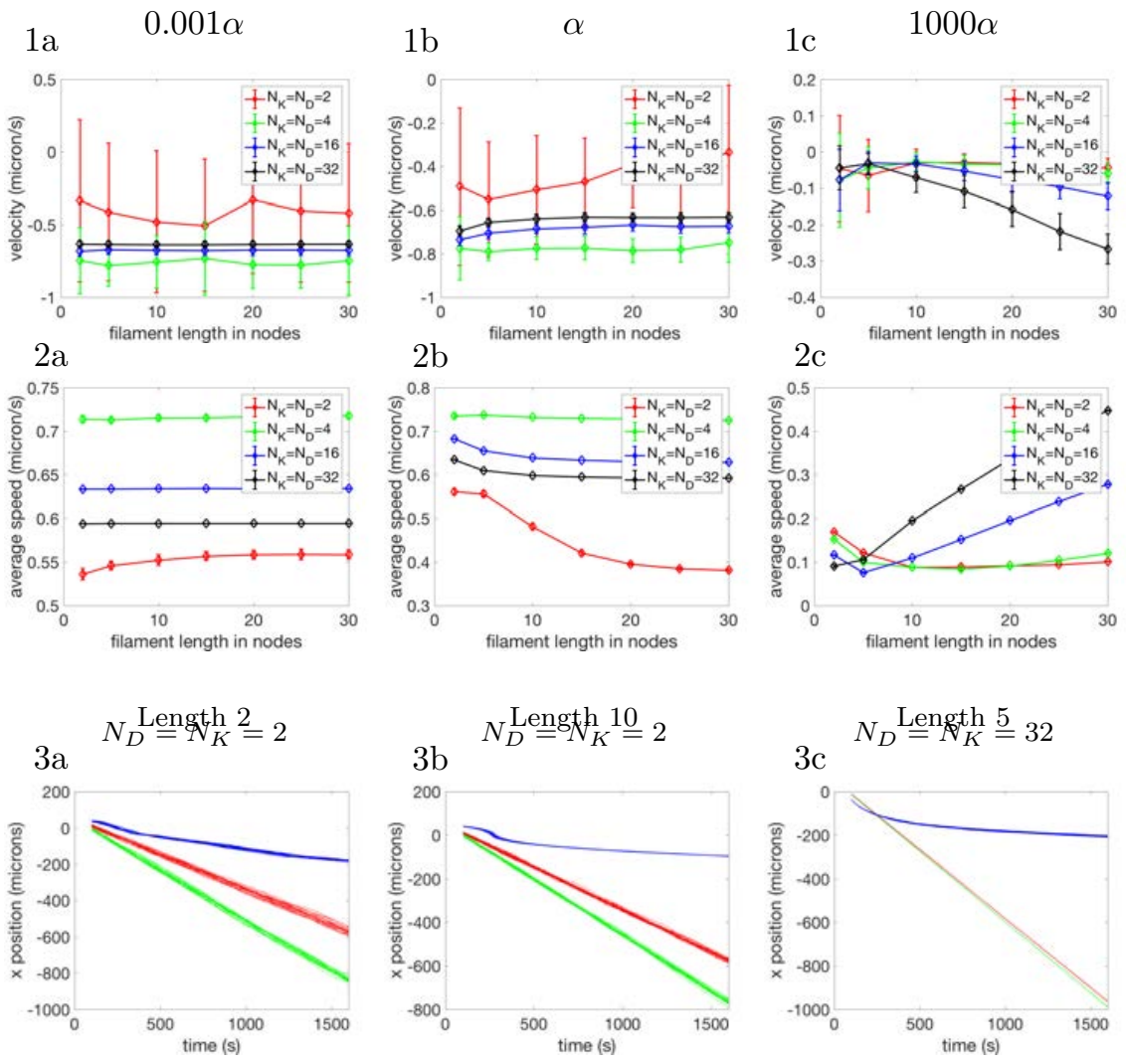

Figure 18: Filament velocity, speed and position when the dynein off rate is catch bond and the kinesin off rate is exponential. In panels 1 the expected velocity of a filament is plotted as a function of filament length. In panels 2 the average speed is plotted. The error bars indicate the standard deviation. In panels $1(\mathrm{a})$ and $2(\mathrm{a})$ the filament elasticity is $0.001 \alpha$, in 1 (b) and 2(b), $\alpha$ and in 1(c) and 2(c), 1000 $\alpha$. The different curves within each frame are for constant $N_{D}=N_{K}$ with the values given in the legend. Panels 3 show the position of the first node as a function of time for selected realizations. The red lines are simulations of filaments with low spring constant, the green lines normal elasticity, and the blue lines high spring constant. Panel 3(a) is for simulations with filament length 2 and $N_{D}=N_{K}=2$, panel 3(b) with filament length 10 and $N_{D}=N_{K}=2$, and panel 3(c) with filaments of length 5 and $N_{D}=N_{K}=32$. In these simulations the parameters are the same as figure6 except the off rates. 

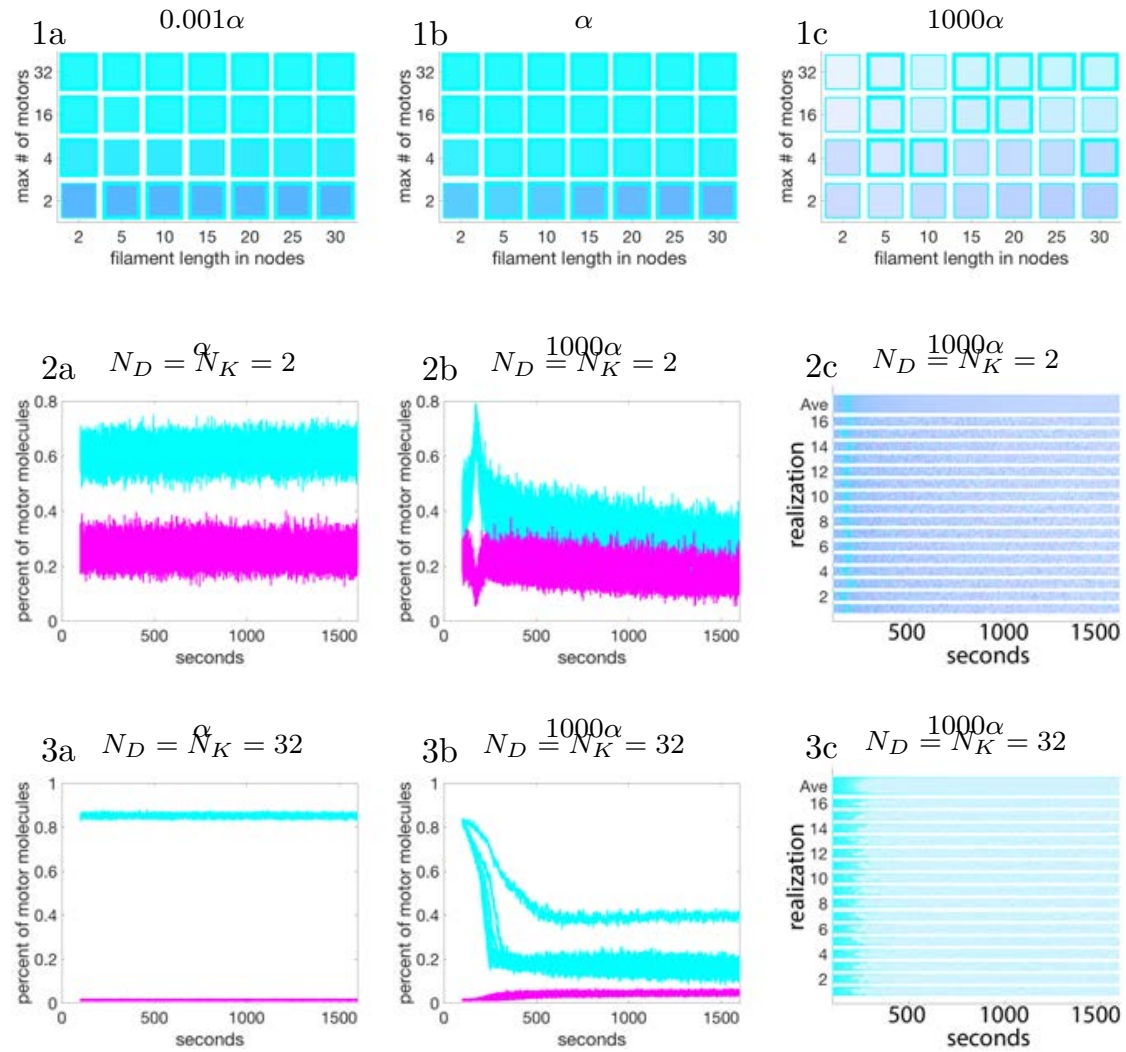

color code

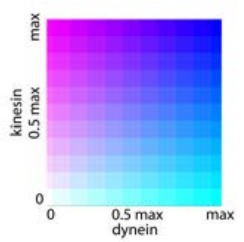

Figure 19: Average number of motor molecules for simulations with dynein off rate catch bond and kinesin off rate exponential. Panels 1 are the same as those shown in figure 14 The panels 2 and 3 show the number of dynein and kinesin molecules as they change with time for simulations with filaments of length 15 nodes. Thus there are 15 lines plotted in each color in panels 2(a), 3(a), 2(b), and 3(b). Panels 2(a) and 3(a) are for filaments with spring constant $\alpha$ and 2(b), 3(b), 2(c), and 3(c) are for $1000 \alpha$. Panels 2 have $N_{D}=N_{K}=2$ and 3 have $N_{D}=N_{K}=32$. The color key is in the bottom left corner. 
the variation in the magenta and cyan lines in the first column decreases as the maximum number of motor molecules increases. In panel 3(b) the separation

950 of the cyan lines shows that interior nodes have less dynein than exterior nodes (this can also be seen in the top color bar of panel 3(c)). The results for low filament stiffness are similar to those with normal filament elasticity and the initial conditions of the nodes do not seem to affect the results. For stiffer filaments, the system exhibits a transient behavior, it moves towards a high 955 dynein value and low kinesin value (what appears to be a the steady state for the lower elasticities) but then the dynein decreases over time. The peak in the dynein moves to the left as the length of the filament increases. 

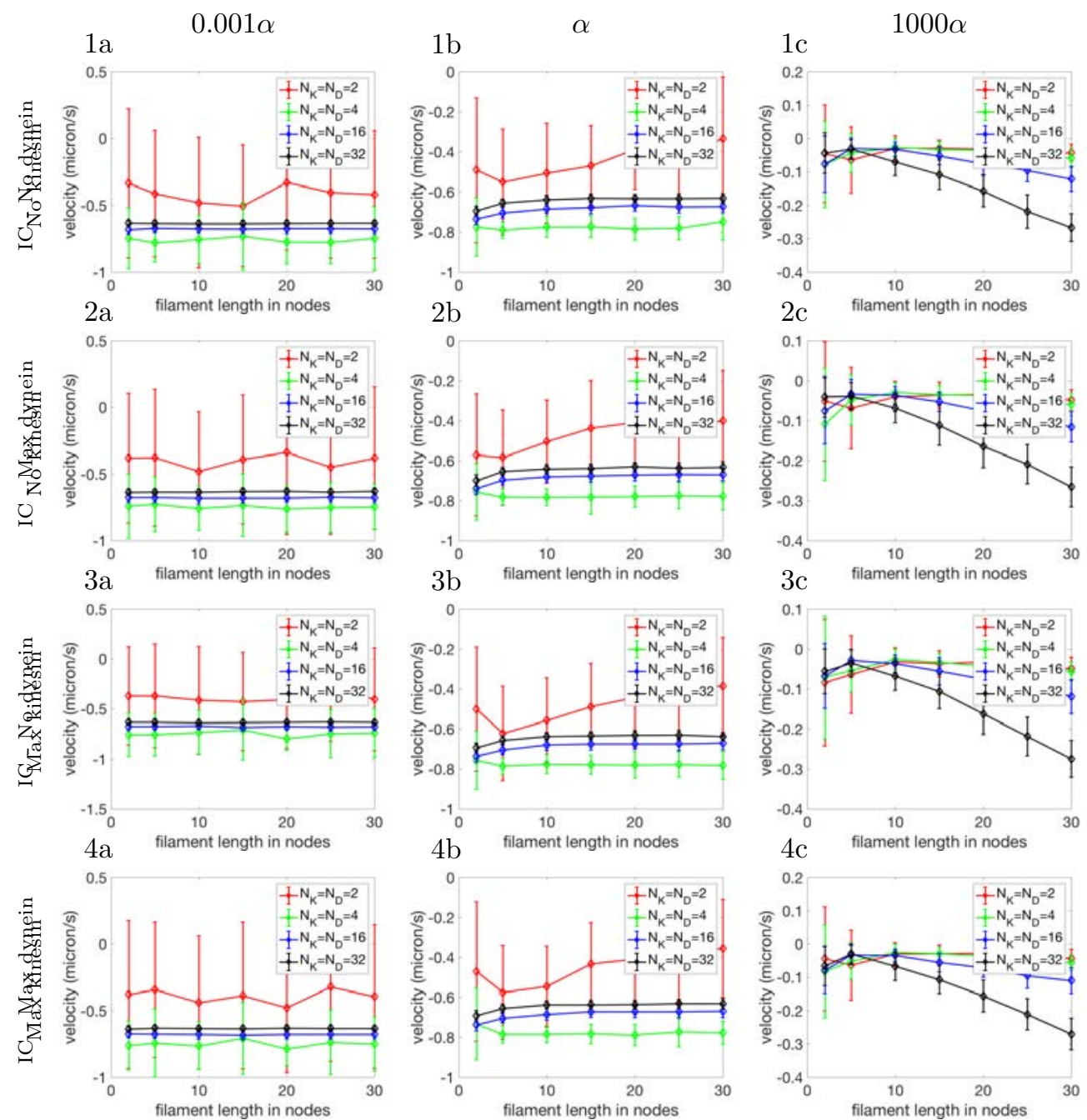

Figure 20: Expected velocity of a filament is plotted as a function of filament length when the dynein off rate is catch bond and the kinesin off rate is exponential. The error bars indicate the standard deviation. The initial conditions change for each row and the filament elasticity varies from low to high with the columns as in figure 15 The different plots within each frame are for constant $N_{D}=N_{K}$ with the values given in the legend. 

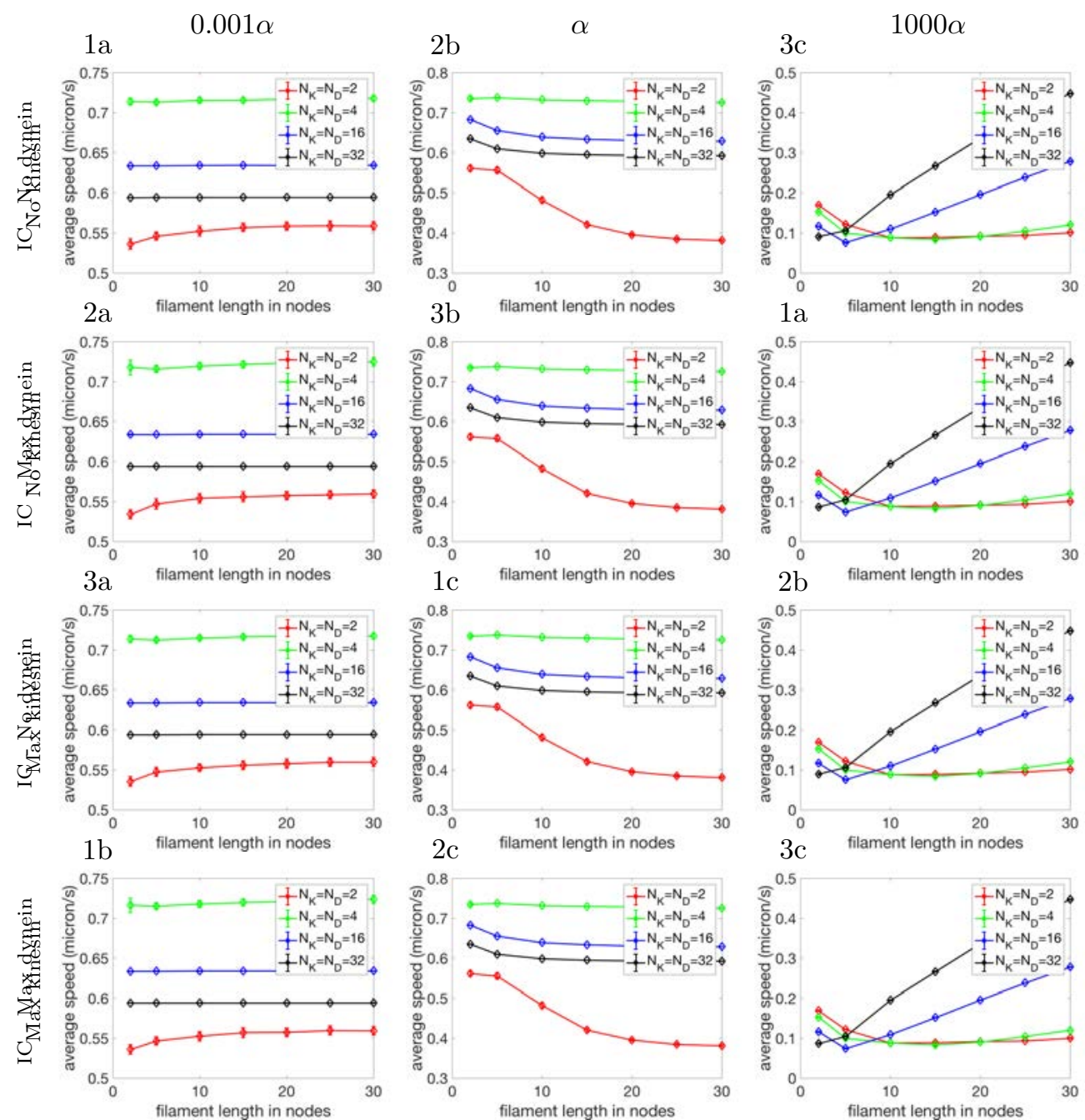

Figure 21: Expected speed of a filament is plotted as a function of filament length when the dynein off rate is catch bond and the kinesin off rate is exponential. The error bars indicate the standard deviation. The initial conditions change for each row and the filament elasticity varies from low to high with the columns as in figure 16 The different plots within each frame are for constant $N_{D}=N_{K}$ with the values given in the legend. 

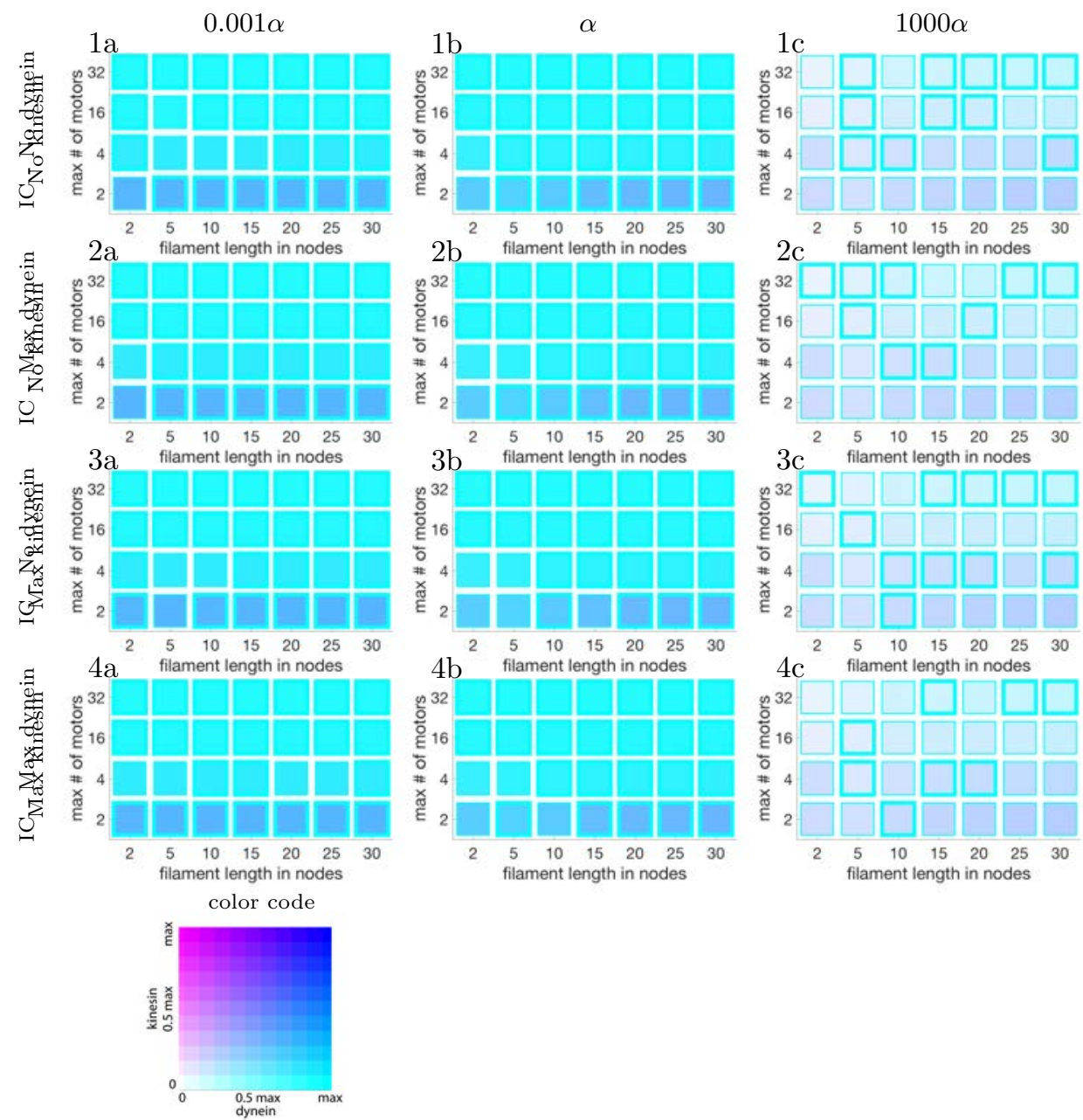

Figure 22: The final number of dynein and kinesin averaged over realizations and nodes when the dynein off rate is catch bond and the kinesin off rate is exponential. The border color indicates which motor molecule is more abundant. If the border is thick it indicates the number of motor molecules is near a steady state value. If the border is thin the number of motor molecules is still changing. The initial conditions change for each row and the filament elasticity varies from low to high with the columns as in figure 17. Cyan depicts dynein only, magenta kinesin only, and blue means equal amounts of each motor molecule. The color key is shown in bottom left corner. The time shown is 1600 seconds. 


\subsection{Off Rates - Dynein Exponential, Kinesin Catch Bond}

In this section all the simulations have the exponential off rate for dynein

960 atch bond off rate for kinesin.

The velocity plots of the filaments shown in figure 23 panels 1 are similar to the velocity plots where the off rate for dynein is catch bond and kinesin is exponential. The initial conditions for these simulations are no motor molecule. The big difference is that kinesin dominates and the velocities are positive. play much of a role. The velocity increases and then decreases with maximum number of motor molecules except in the stiffest filaments where it increases for long filaments.

In figure 23 panels 2 the speed of the filaments is plotted. The graphs are 970 very similar to those in figure 18 . For the least stiff filaments the length does not seem to affect the speed but the number of maximum motor molecules does with the value of 16 giving the maximum speed (in the previous case the maximum speed occurred at 4). Filaments with normal elasticity have speeds which decrease with length and change with the maximum number of motor 975 molecules. Filaments which are the stiffest have increasing speed with length and for long filaments increase speed with increasing maximum motor molecules. The position plots for these simulations panels 3 are comparable to those in figure 18 and look the same except they have positive slopes and the transients in the stiffest filaments are not as noticeable.

Figure 24 panels 2 and 3 are similar to those panels in figure 19 except the kinesin is greater than the dynein. Again for the stiffest filaments the first and last nodes have more molecules attached than the interior nodes. Filaments with the lowest spring constant are similar to those with the normal spring constant. In the stiffest filaments the same peak as before is not observed. It may come in the first 100 seconds which are not plotted.

For each set of parameters and initial conditions the expected velocity of the filament is shown in figure 25 the speed in figure 26, and the final number of motor molecules in figure 27. The filament elasticity varies with the columns and the initial conditions vary with the rows. It is clear from figure 27 that kinesin dominates.

In figure 26 the speed of the filaments is plotted. The graphs are very similar to those in figure 21. For the least stiff filaments (panels (a)) the length does not seem to affect the speed but the number of maximum motor molecules does with the value of 16 giving the maximum speed (in the previous case the maximum 995 speed occurred at 4). Filaments with normal elasticity (panels (b)) have speeds which decrease with length and change with the maximum number of motor molecules. Filaments which are the stiffest (panels (c)) have increasing speed with length and for long filaments increase speed with increasing maximum motor molecules.

Unlike the previous cases when the steady state criterion is change to less than 5 percent in figure 27 there are 15 squares that do not meet it. Twelve are for all initial conditions with the stiffest filaments (panels (c)). They are 

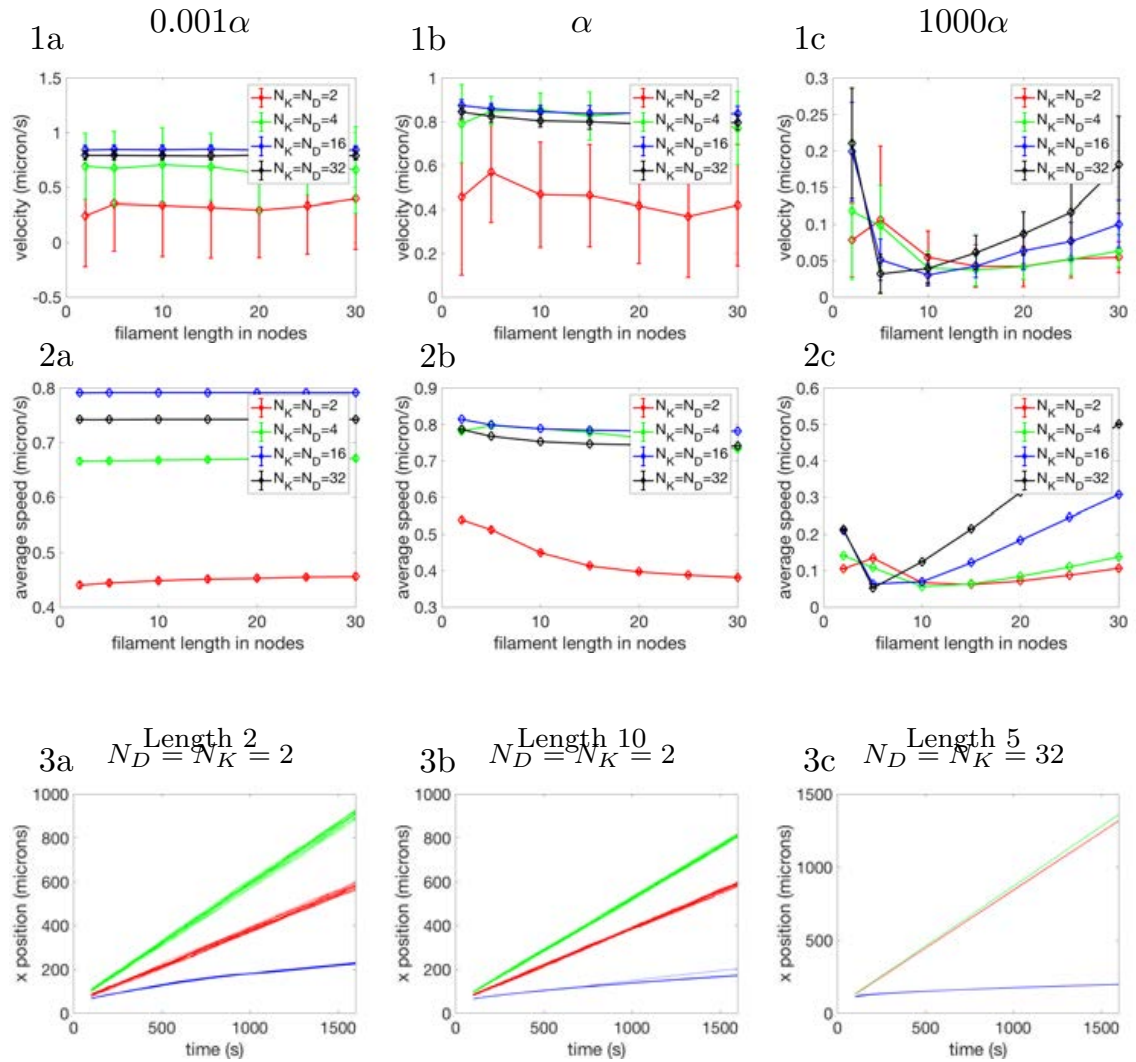

Figure 23: Filament velocity, speed and position when the dynein off rate is exponential and the kinesin off rate is catch bond. In panels 1 the expected velocity of a filament is plotted as a function of filament length. In panels 2 the average speed is plotted. The error bars indicate the standard deviation. In panels $1(\mathrm{a})$ and $2(\mathrm{a})$ the filament elasticity is $0.001 \alpha$, in 1 (b) and 2(b), $\alpha$ and in 1(c) and 2(c), 1000 $\alpha$. The different curves within each frame are for constant $N_{D}=N_{K}$ with the values given in the legend. Panels 3 show the position of the first node as a function of time for selected realizations. The red lines are simulations of filaments with low spring constant, the green lines normal elasticity, and the blue lines high spring constant. Panel 3(a) is for simulations with filament length 2 and $N_{D}=N_{K}=2$, panel 3(b) with filament length 10 and $N_{D}=N_{K}=2$, and panel 3(c) with filaments of length 5 and $N_{D}=N_{K}=32$. In these simulations the parameters are the same as figure6 except the off rates. 

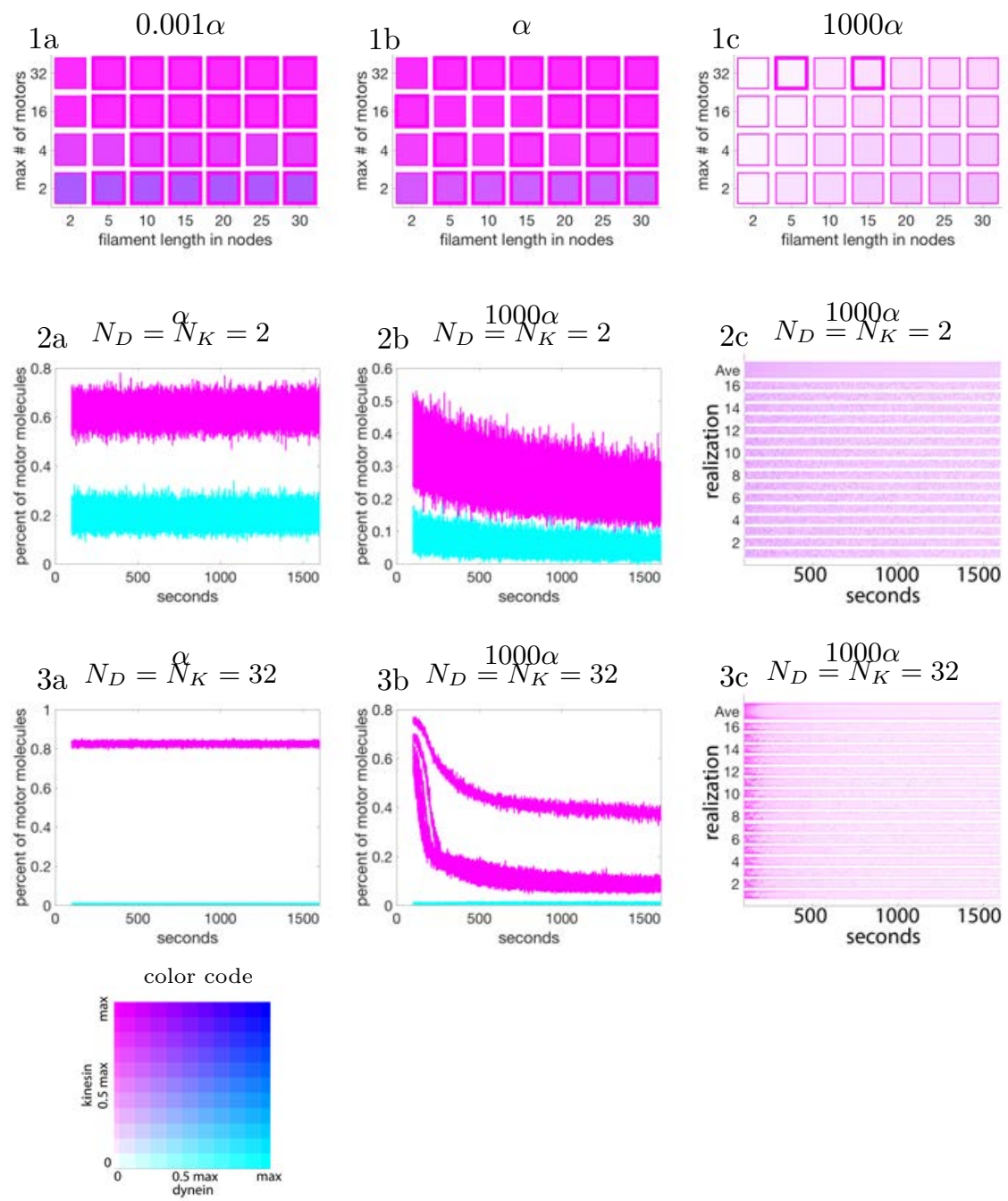

Figure 24: Average number of motor molecules for simulations when the dynein off rate is exponential and the kinesin off rate is catch bond. Panels 1 are the same as those shown in figure 19 Panels 2 and 3 show the number of dynein and kinesin molecules as they change with time for simulations with filaments of length 15 nodes. Thus there are 15 lines plotted in each color in panels 2(a), 3(a), 2(b), and 3(b). Panels 2(a) and 3(a) are for filaments with spring constant $\alpha$ and 2(b), 3(b), 2(c), and 3(c) are for $1000 \alpha$. Panels 2 have $N_{D}=N_{K}=2$ and 3 have $N_{D}=N_{K}=32$. The color key is in the bottom left corner. 

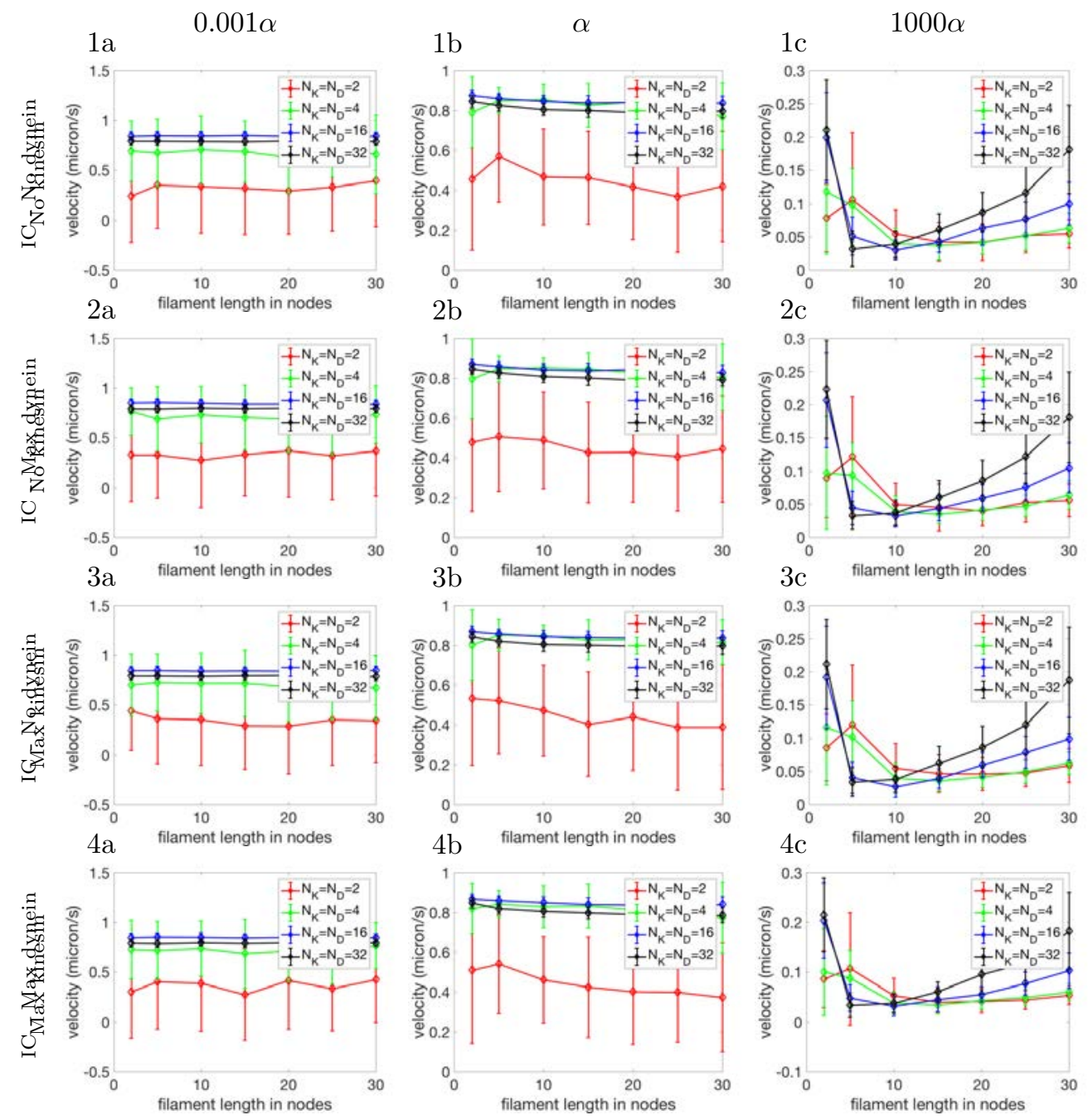

Figure 25: The expected velocity of a filament is plotted as a function of filament length when the dynein off rate is exponential and the kinesin off rate is catch bond.. The error bars indicate the standard deviation. The initial conditions change for each row and the filament elasticity varies from low to high with the columns as in figures 15 and 20 The different plots within each frame are for constant $N_{D}=N_{K}$ with the values given in the legend. 

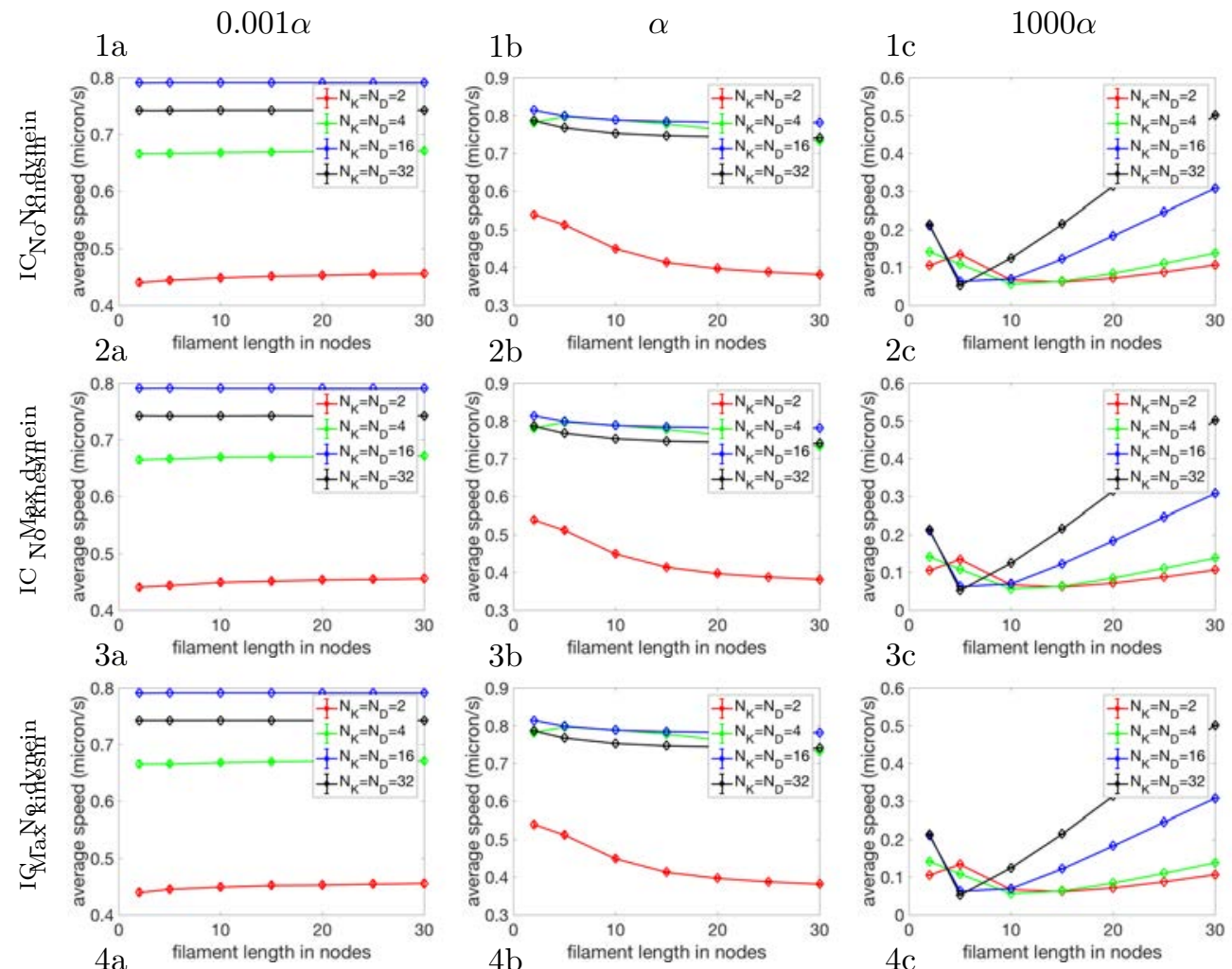

$3 \mathrm{c}$ filament length in nodes
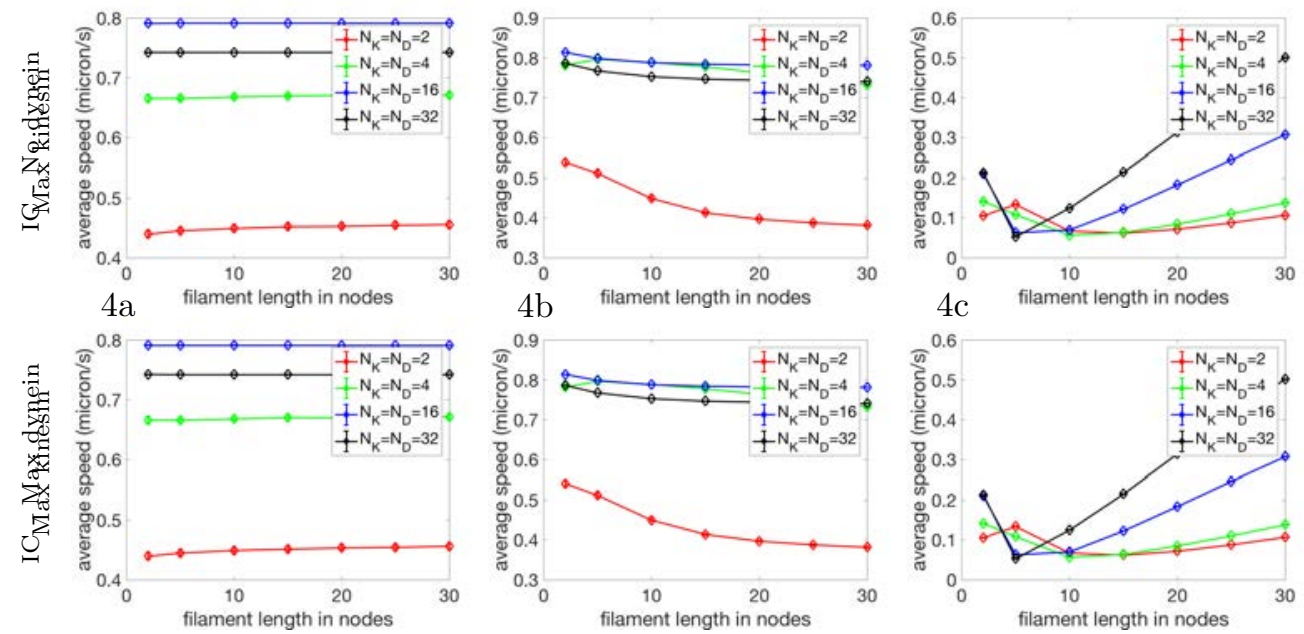

Figure 26: The expected speed of a filament is plotted as a function of filament length when the dynein off rate is exponential and the kinesin off rate is catch bond.. The error bars indicate the standard deviation. The initial conditions change for each row and the filament elasticity varies from low to high with the columns as in figures 16 and 21 The different plots within each frame are for constant $N_{D}=N_{K}$ with the values given in the legend. 
simulations with filament length $2, N_{D}=N_{K}=2$ and 4 , and filament length 30 with $N_{D}=N_{K}=32$. Two of the other three are in panel 4(b), filament 1005 length 2 , and $N_{D}=N_{K}=2$, and 4. The final square is in panel 1(b), filament length 2 , and $N_{D}=N_{K}=4$. 

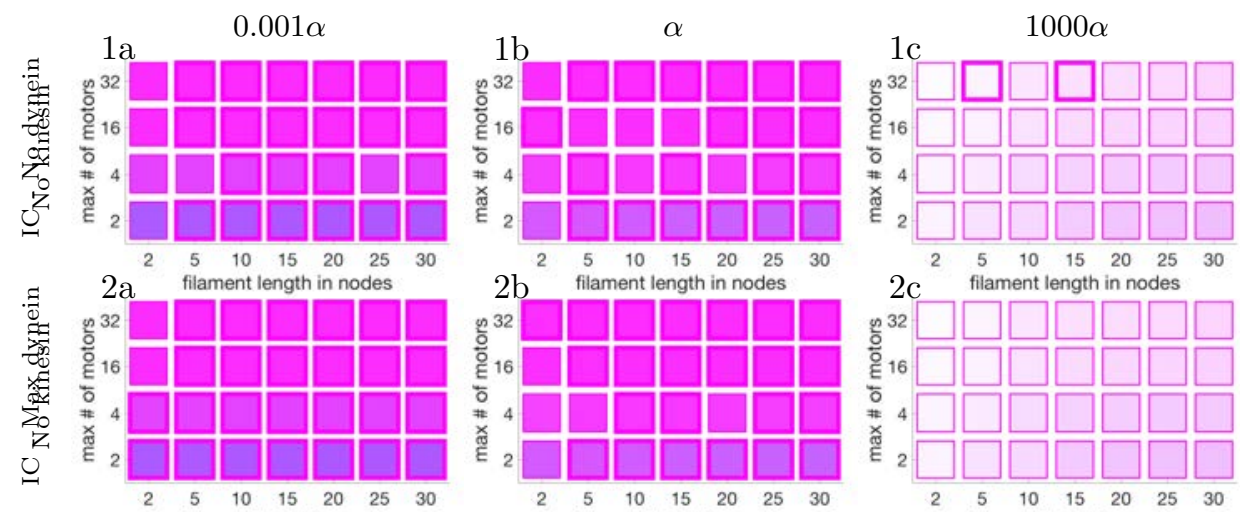

$2 \mathrm{~b} \quad$ filament length in nodes
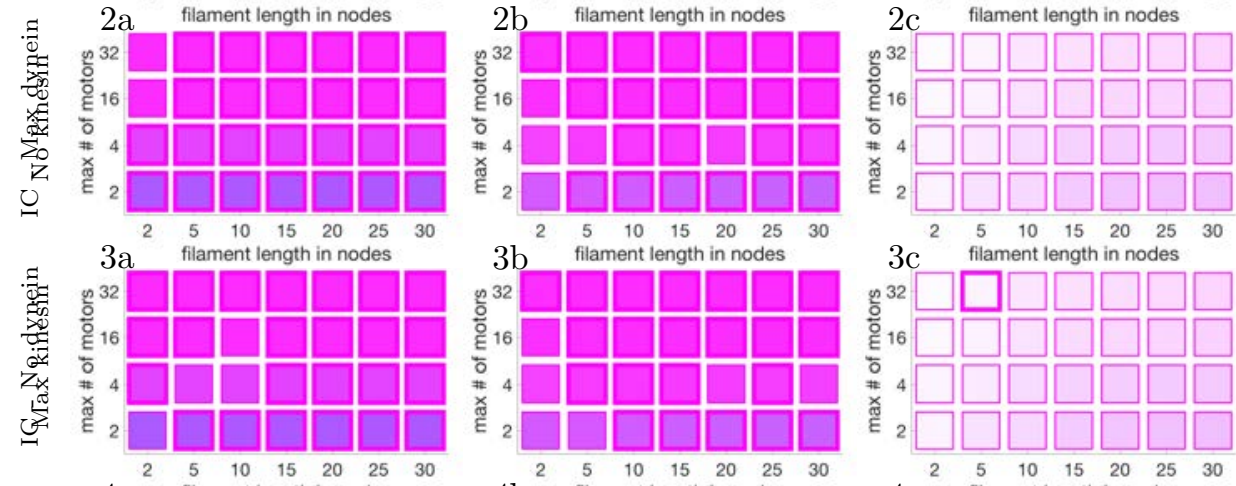

$3 \mathrm{~b} \quad$ filament length in nodes
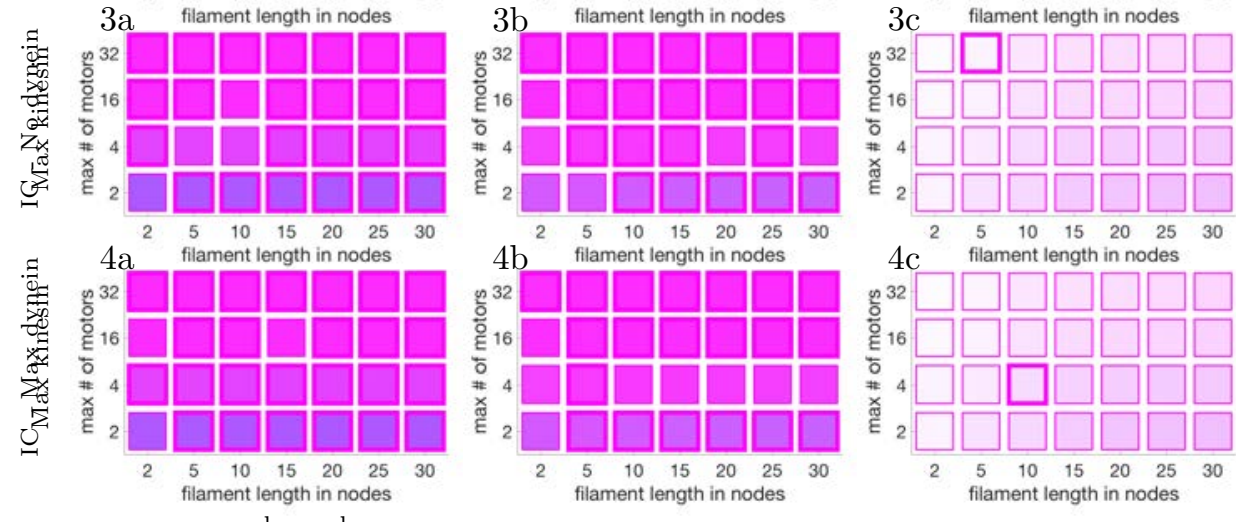

$4 \mathrm{c} \quad$ filament length in nodes
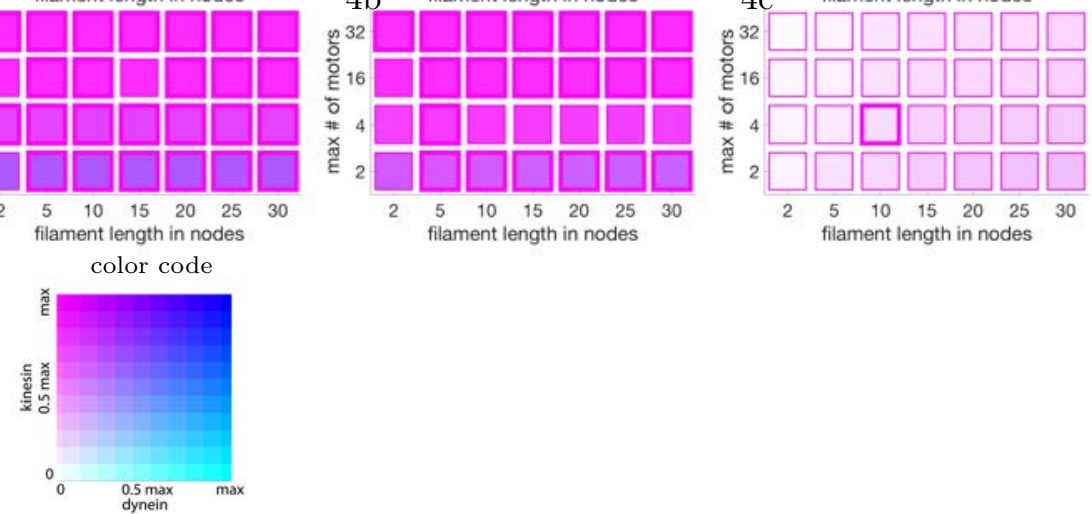

Figure 27: The final number of dynein and kinesin averaged over realizations and nodes when the dynein off rate is exponential and the kinesin off rate is catch bond. The border color indicates which motor molecule is more abundant. If the border is thick it indicates the number of motor molecules is near a steady state value. If the border is thin the number of motor molecules is still changing. The initial conditions change for each row and the filament elasticity varies from low to high with the columns as in figures 17 and 22 Cyan depicts dynein only, magenta kinesin only, and blue means equal amounts of each motor molecule. The color key is shown in the bottom left. The time shown is 1600 seconds. 


\subsection{Off Rates - Dynein Exponential, Kinesin Exponential}

In these simulations both off rates are exponential. Their are three main differences in these simulations compared to the other simulations. The first is

1010 that the initial conditions are important. The second is that more simulations in this set fail the steady state criterion indicating that the system is still not near an equilibrium.

Figure 28 shows the velocity of the filaments. The velocity seems to be independent of the filament length for the low spring constant and normal spring constant simulations. For the very stiff filaments the velocity decreases with increasing filament length (with the exception of filaments of length 2 with 2 maximum motor molecules). Filaments with low maximum motor molecules have both velocities. The stiffest filaments have the lowest magnitude velocities and averages are all positive. For filaments with normal elasticity and low 1020 stiffness the velocity of the filaments with the most motor molecules is determined by the initial conditions. If the filaments start with maximum dynein they have a negative velocity and if they start with no dynein they have a positive velocity. The magnitude of the velocity increase as the number of motor molecules increase except for the stiffest filaments. In that case filaments lose motor molecules as time goes on and the velocities are naturally lower.

In figure 29 the speed of the filaments are plotted. For filaments with low spring constants (panels (a)) the speed increases with filament length. With filaments that have normal elasticity (panels (c)) the speed decreases with length, and with the stiffest filaments (panels (c)) the speed goes down then up (with $1030 \quad$ one exception $\left.N_{D}=N_{K}=2\right)$.

Figure 30 shows the number of motor molecules attached to each node. In this figure the first and last column are for filaments with low spring constant and high spring constant respectively. The middle two columns are for filaments wth normal elasticity. Column two, three, and four have the maximum number of 1035 motor molecules as 2, 4, and 16 respectively. The first column is for filaments of length 15 nodes and for initial conditions maximum dynein and no kinesin. The normal initial conditions for these simulations of no motor molecules attached, shows a different behavior, see figure 32 Panels 1(a) and 2(a) show that longer filaments with low stiffness have a uniform behavior for all the filaments. This can also be seen in panels (b)) yet there each filament seems to have short periods of all one color which can rapidly change. Panels (c)) show that with a maximum of 4 motor molecules the filaments clearly show a switching behavior with periods of time where one molecule dominates and then it switches so the other motor dominates. As the filaments get longer the switching decreases.

1045 In the case of low stiffness, for high max motor molecules (figure 32 panels $3(\mathrm{a})$ and 4(a)) one or the other motor molecule dominates in all realizations. As the maximum motor molecules decreases, each filament has a greater mixture of dynein and kinesin. For filaments with normal stiffness and high motor molecule maximum $(16,32)$ the filaments have one motor molecule which dominates in a fashion similar to low stiffness (graphs are not shown as they are similar to the first column in figure 30 . For a maximum of 4 motor molecules for each motor 

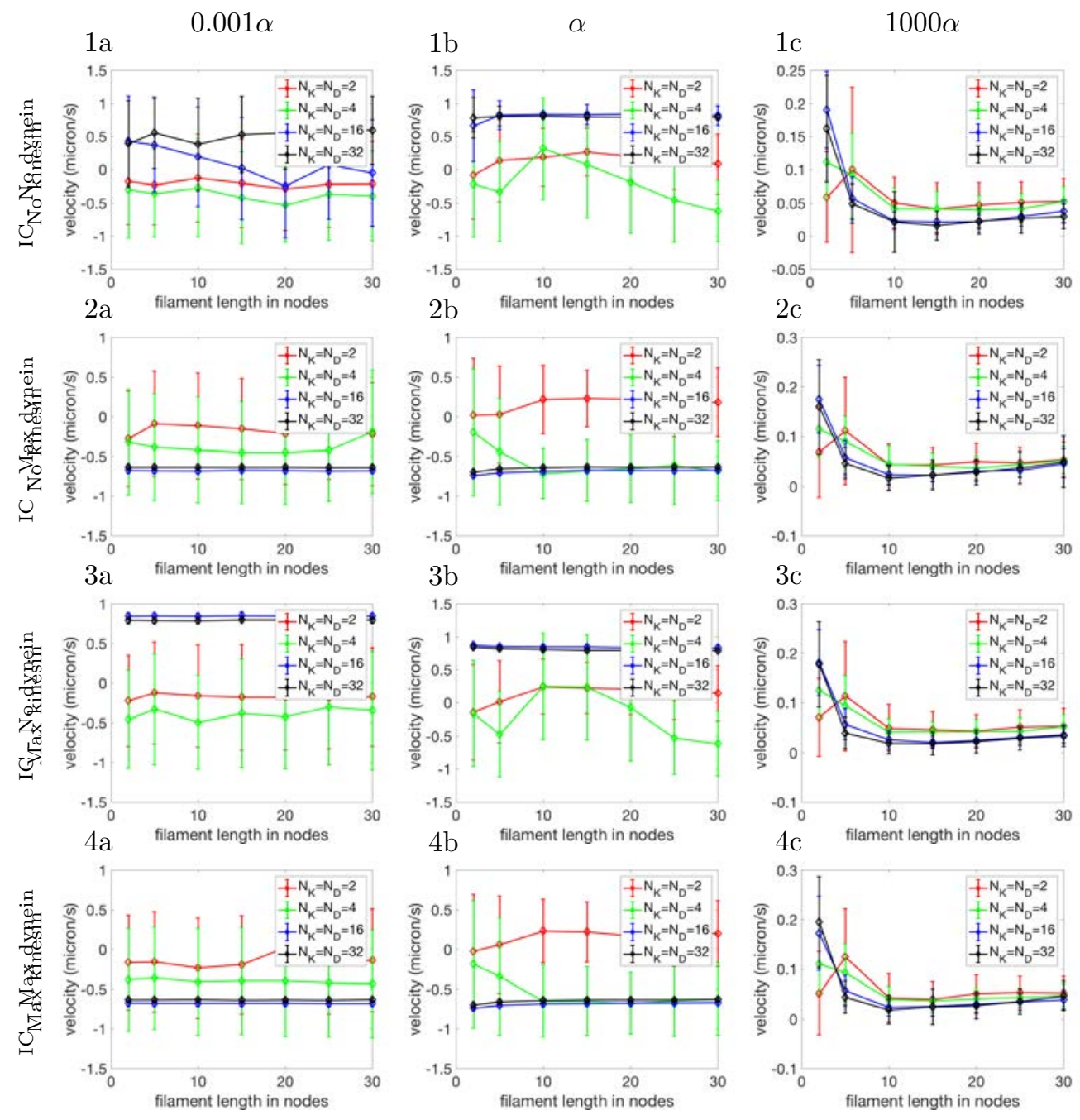

Figure 28: The expected velocity of a filament is plotted as a function of filament length when both off rates are exponential. The error bars indicate the standard deviation. The initial conditions change for each row and the filament elasticity varies from low to high with the columns as in figure 7 The different plots within each frame are for constant $N_{D}=N_{K}$ with the values given in the legend. This figure can be compared with figures 1520 and 25 

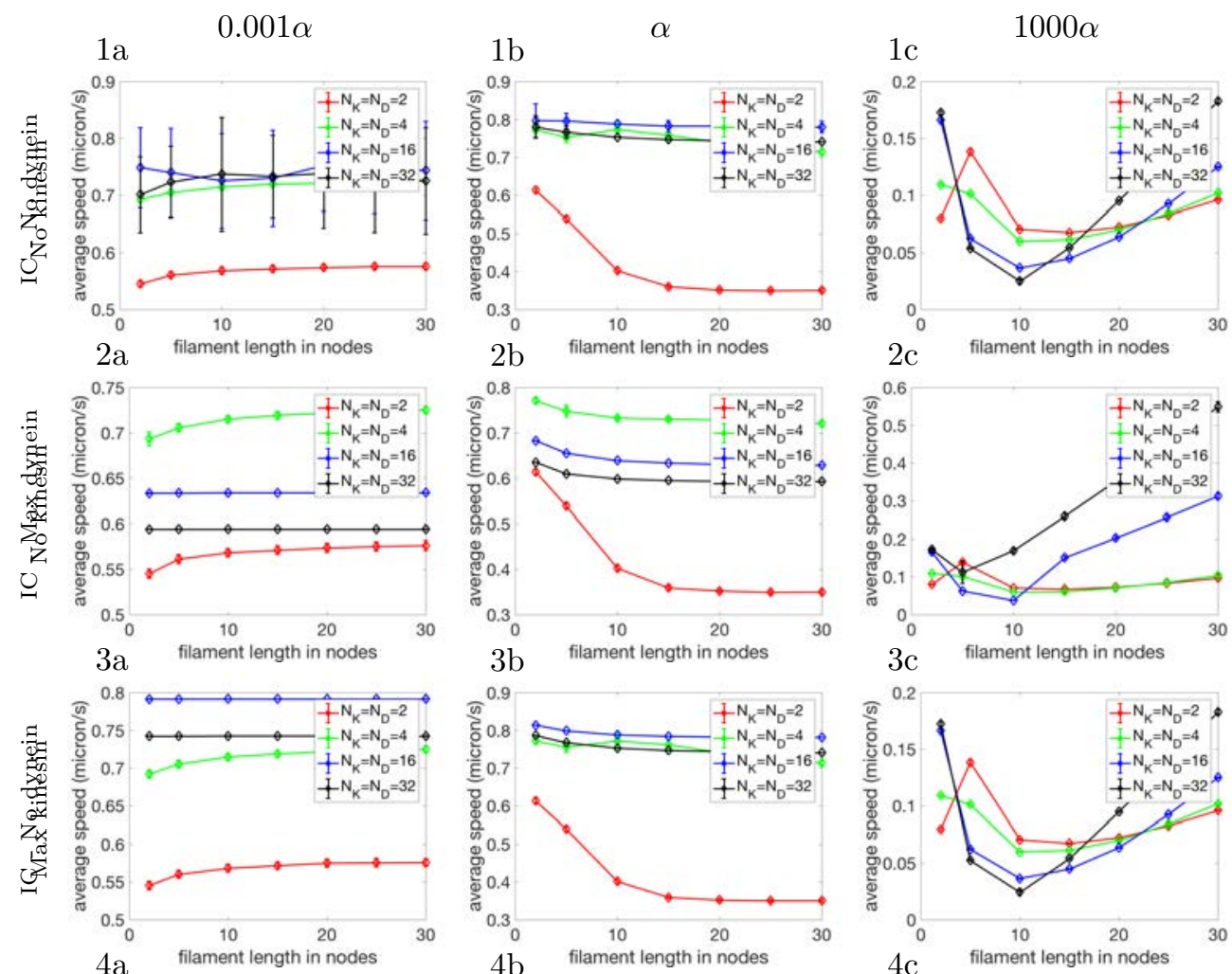

$3 \mathrm{c}$ filament length in nodes
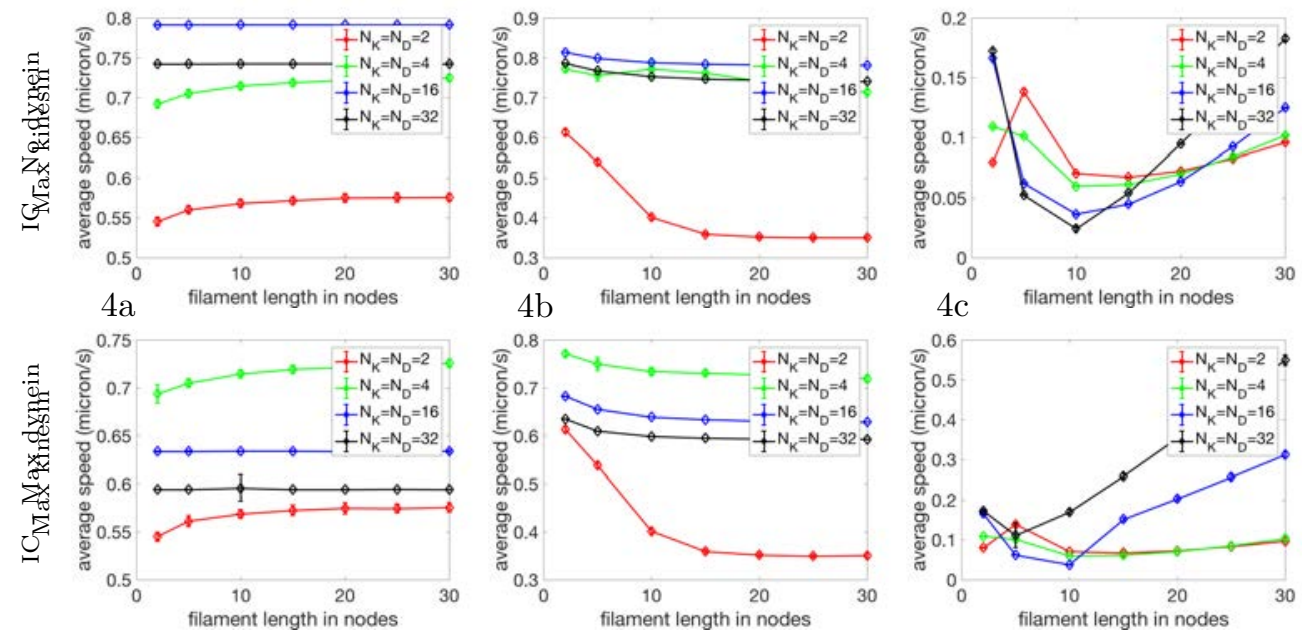

Figure 29: The expected speed of a filament is plotted as a function of filament length when both off rates are exponential. The error bars indicate the standard deviation. The initial conditions change for each row and the filament elasticity varies from low to high with the columns as in figure 7 The different plots within each frame are for constant $N_{D}=N_{K}$ with the values given in the legend. This figure can be compared with figures 16 21. and 26 
type, the switching behavior is seen with greater frequency for short filament lengths (panel 1(c)). When the maximum motor molecules is 2 (panels (b)), the switching behavior has a high frequency (panels 1(b) and 2(b)) for short filaments and disappears as the filaments get longer than 5 nodes (panels 3(b) and $4(\mathrm{~b}))$.

Filaments with the highest spring constant (panels (d)) lose attached motor molecule as time progresses and the interior nodes lose the bound motors first. In the end the two end nodes seem to move the filament with minimal contribution from the interior nodes (panels 3(d) and 4(d)).

Figure 31 shows the normalized number of motor molecules for each node. The simulations are the same as those shown in figure 30 Observe the simulations shown in figure 31 panels 1(c), 2(c), 3(c), and 4(c) have longer transient than most other simulations. Figure 32 shows a variety of possible dynamics obtained when both off rates are exponential and the lowest stiffness: in panel (A), the local tug of war persists at each node and there is no coordination between nodes along the filaments; in panel (B), nodes are coordinated along the filaments and the local tug of war is resolved, each node has reach a steady state. The stochastic nature of the simulations is apparent in panel (A) with 1070 the number of motor molecules changing over time for an individual node but the average number over all the nodes remaining constant. This is a common structure for simulations with a low maximum number of motor molecules. The solid colors in panel (B) are common for filaments with a higher maximum number of motor molecules. In panels (C) and (D), nodes are coordinated along the

1075 filaments; however, in some cases there exist travelling waves along the filament as seen by a sequential switching of adjacent nodes between two steady states of the number of motors attached. Sometimes switching affects only adjacent nodes within a filament and propagates along the filament and then vanishes; this switching does not propagate to all the nodes and does not induce a global change of direction of the filament. Hence, symmetry in off rates allows competition amongst motors; even if a filament overall moves in one direction it still is subject to a local tug-of-war at nodes. Yet in some cases the switch is permanent and propagates from node to node changing the global behavior of the filament. The structure in panels (C) and (D) is less common, although

1085 variation in time is not as uncommon. As the filament length increases the structure is more complex and it takes a longer time for the average to approach a steady state value. The overall main direction of filaments does not depend on initial conditions in the case of both catch bond off rates but on filament properties (stiffness and length). In the case of both exponential off rates and low or normal spring constants, the initial condition of dynein is more important in determining whether the filament will end up with more dynein or kinesin. Although the final state does depend on the filament length and the maximum number of motor molecules allowed. When the initial dynein is low the filament will end up with more kinesin if the maximum number of motor 1095 molecules is larger. When the intial dynein is high the filament will end up with more dynein except for some cases with low maximum motor molecules. 


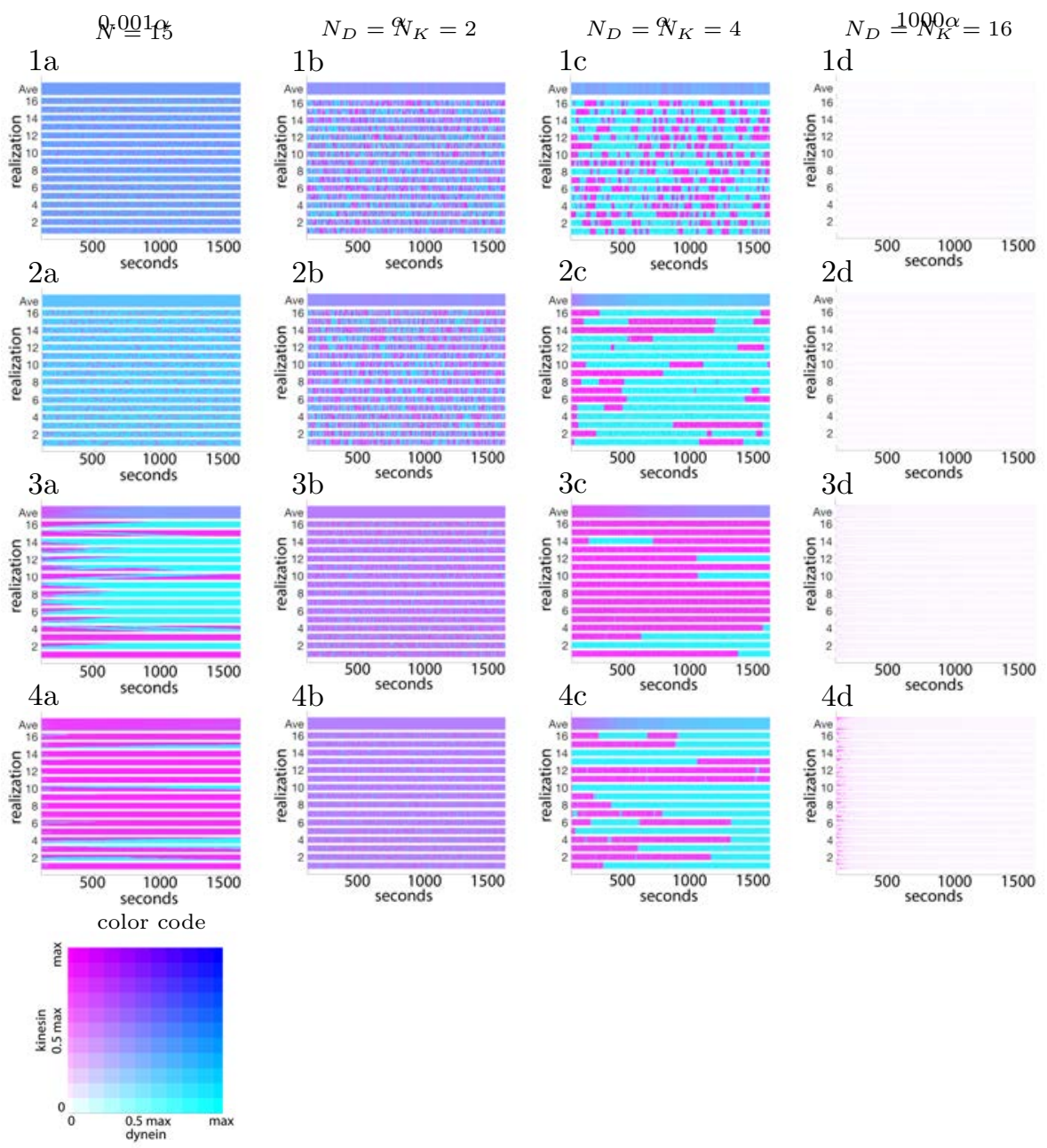

Figure 30: Plots of the number of motor molecules attached to nodes as function of time when both off rates are exponential. In panels (a) the filament stiffness is low, in panels (b) the filament stiffness is normal and panels (c) the filament stiffness is high. In panels (b), (c), and (d) $N_{D}=N_{k}=2,4$, and 16 respectively and the filament length changes with the row from $2,5,15$, to 25 nodes respectively. In panels (a) the filament length is fixed at 15 nodes and $N_{D}=N_{K}$ changes from 2, 4, 16, and 32 as the rows change. Switching behavior is seen in panels 1 (b), 2(b), and (c). 

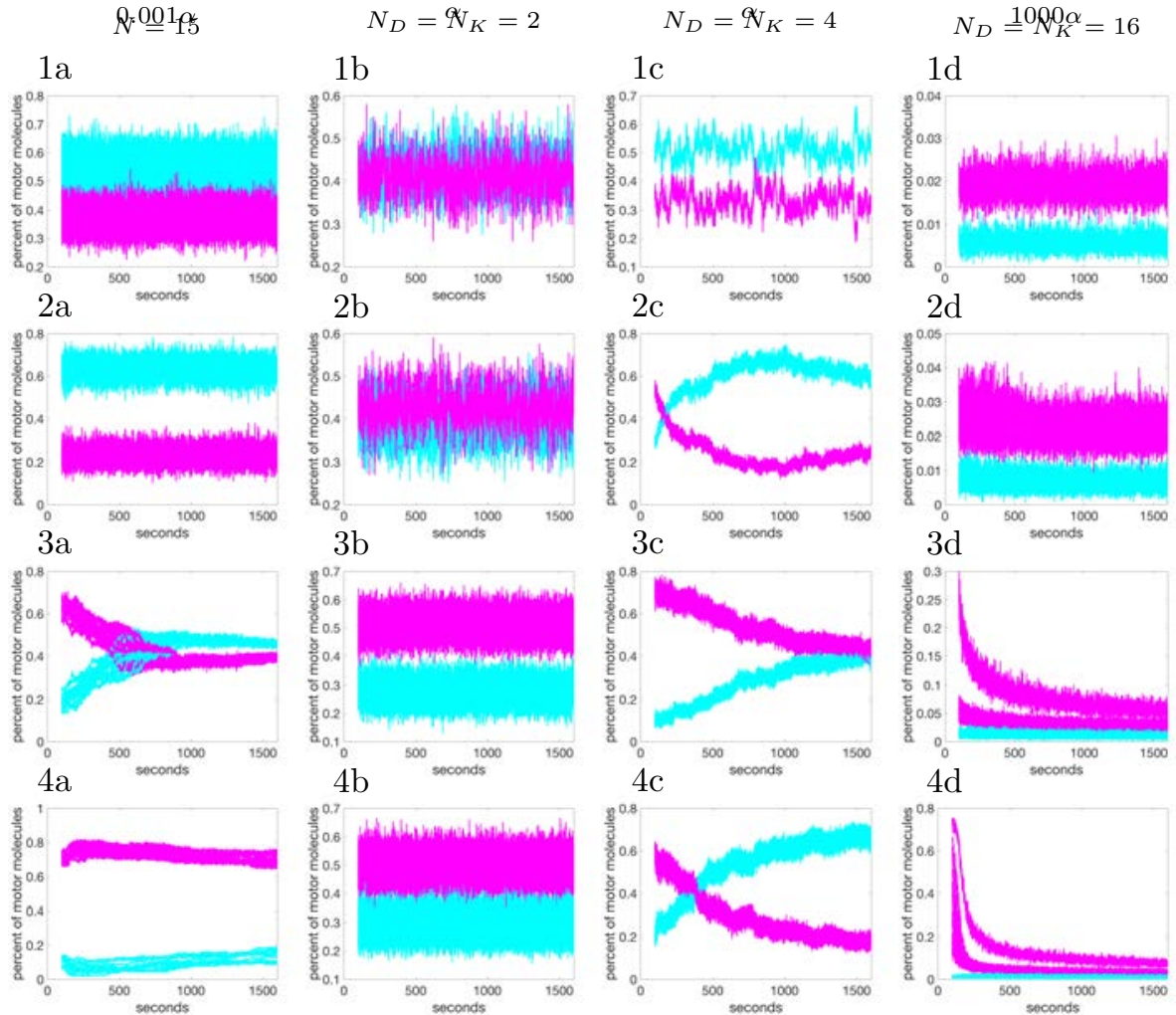

Figure 31: Plots of the average percent of dynein and kinesin per node are shown as a function of time when both off rates are exponential. These are the same simulations for figure 30 In panels (a) the filament stiffness is low, in panels (b) and (c) the filament stiffness is normal and in panels (d) the filament stiffness is high. In panels (b), (c), and (d) $N_{D}=N_{k}=2,4$, and 16 respectively and the filament length changes with the row from $2,5,15$, to 25 nodes respectively. In panels (a) the filament length is fixed at 15 nodes and $N_{D}=N_{K}$ changes from $2,4,16$, and 32 as the rows. 

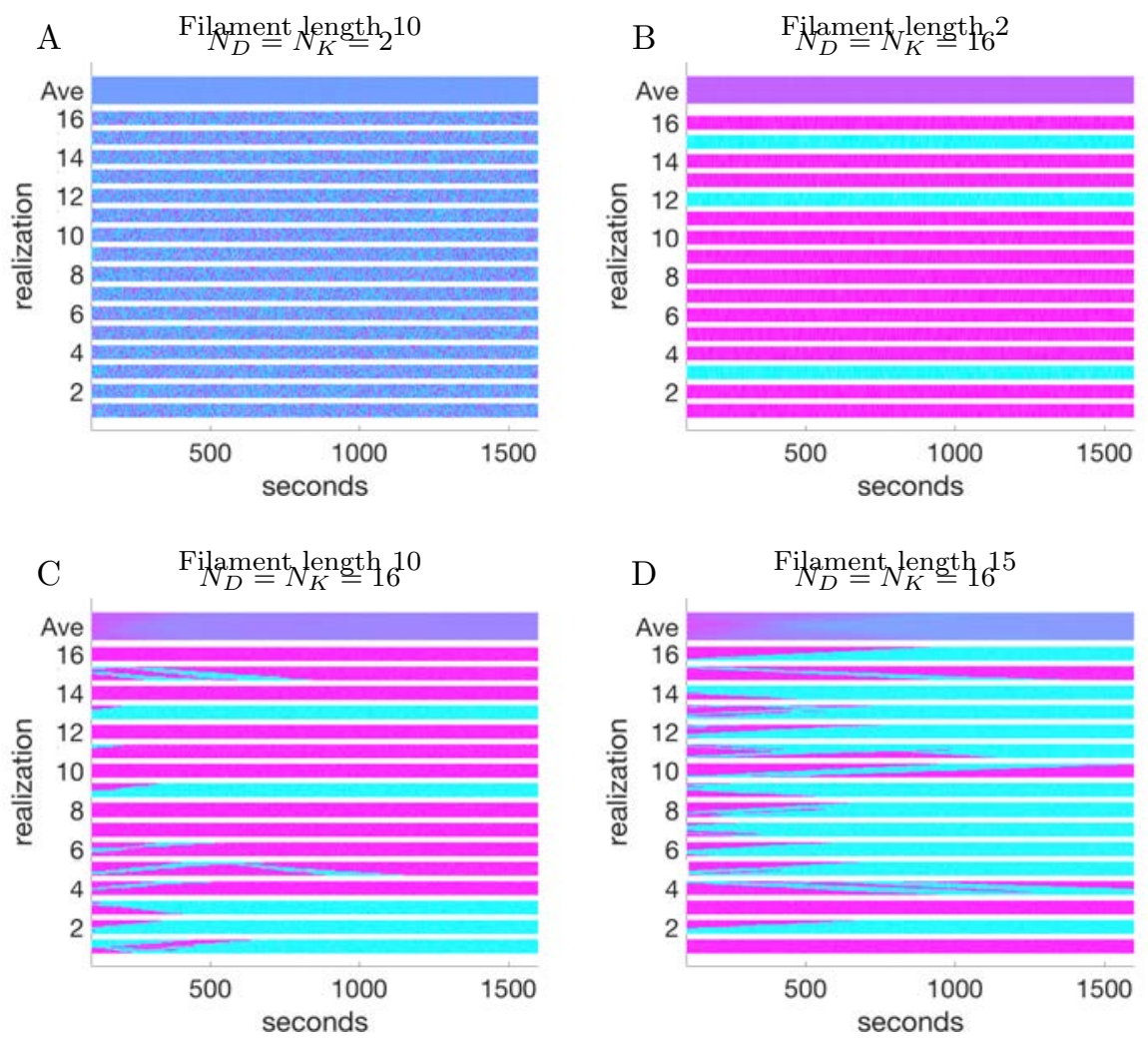

Figure 32: The number of dynein and kinesin changes with respect to time for each node when both off rates are exponential. Initial conditions are set at zero for both dynein and kinesin. In A filament length is 10 nodes and $N_{D}=N_{K}=2$. In B-D $N_{D}=N_{K}=16$, and filament length is 2,10 , and 15 nodes respectively. For these simulations the filament stiffness is low, $0.001 \alpha$. 
Internode distance $0.04 \mu \mathrm{m}$

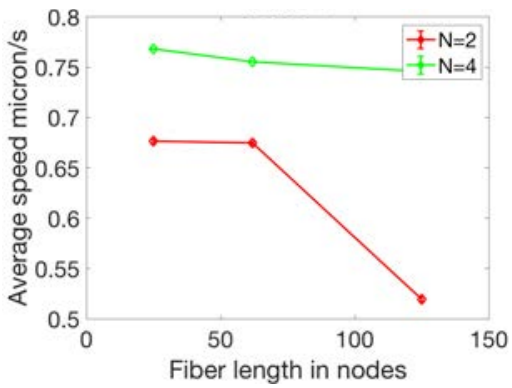

Internode distance $0.5 \mu \mathrm{m}$

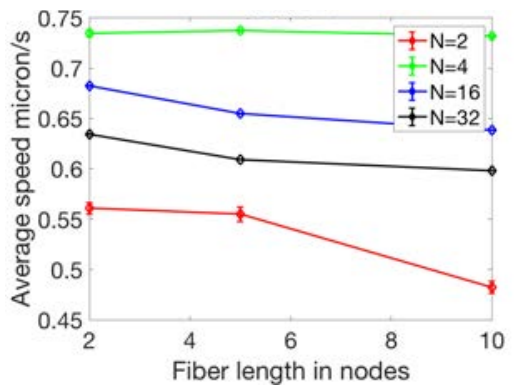

Figure 33: Filament speed is plotted as a function of number of nodes where the dynein off rate is catch bond and the kinesin off rate is exponential. The left panel shows filaments where the internode distance is $0.04 \mu \mathrm{m}$ and the right panels shows simulations with $0.5 \mu \mathrm{m}$. The initial conditions are the maximum number of motors of each type allowed initially attached.

\subsection{Simulations with Short Internode Distance}

Changing the internode distance did not affect the qualitative features of the simulations. In figure 33 the filament speeds for simulations where the internode distance is $0.04 \mu \mathrm{m}$ (about the length of one ULF) is compared to simulations already shown where the internode distance is $0.5 \mu \mathrm{m}$ for the case where the detachment rates are catch bond for dynein and exponential for kinesin and the initial conditions are the maximum number of motors of each type initially attached to each node. The filaments on the left have length $1 \mu \mathrm{m}$ (25 nodes),

${ }_{1105} 2.48 \mu \mathrm{m}$ (62 nodes), and $5 \mu \mathrm{m}$ (125 nodes). (For each node we assume there is a length of filament as opposed to a length of filament between each node.) The maximum number of dynein or kinesin per filament for those in the left panel are 50,124, and 250; and 100, 248, and 500 for the maximum per node of 2 and 4 respectively. In the right panel the total filament lengths are $1 \mu \mathrm{m}$ ( 2 nodes), $2.5 \mu \mathrm{m}$ ( 5 nodes), and $5 \mu \mathrm{m}$ (10 nodes). The maximum number of dynein or kinesin per filament are 4,10 , and 20;8, 20, and 40;32, 80, and 160; and 64,160 , and 320 for those with a maximum of $2,4,16$, and 32 per node respectively. The qualitative behavior of the speed is the same when comparing the maximum per node simulations.

1115 In figure 34 the average number of motor molecules attached to the fibers at the end of the simulation are shown. The left panel shows simulations where the internode distance is $0.04 \mu \mathrm{m}$, whereas the middle and right panels show simulations with the internode distance $0.5 \mu \mathrm{m}$. Again the qualitative features are the same. The simulations with a shorter internode distance are similar to those where the max number of motors per node is the same. 


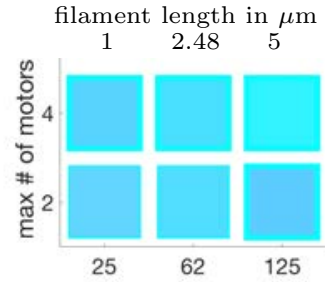

filament length in nodes

color code

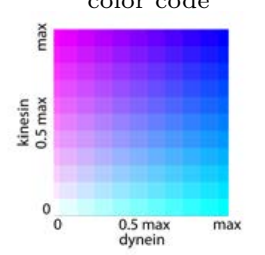

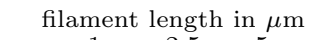

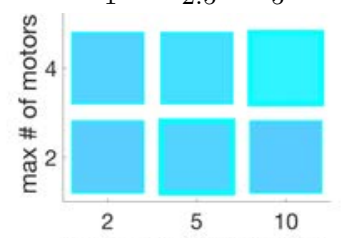

filament length in nodes

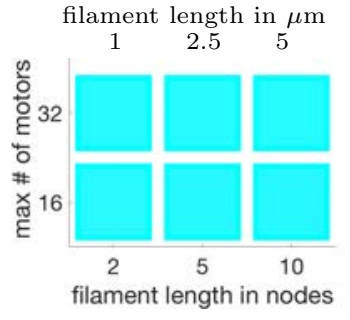

filament length in nodes

Figure 34: The final number of dynein and kinesin averaged over realizations and nodes when the dynein off rate is catch bond and the kinesin off rate is exponential. The left panel shows filaments where the internode distance is $0.04 \mu \mathrm{m}$ and the middle and right panels show simulations with $0.5 \mu \mathrm{m}$. The border color indicates which motor molecule is more abundant. If the border is thick it indicates the number of motor molecules is near a steady state value. If the border is thin the number of motor molecules is still changing. The initial conditions are the maximum number of motors of each type allowed initially attached. Cyan depicts dynein only, magenta kinesin only, and blue means equal amounts of each motor molecule. The color key is shown in the bottom left. The time shown is 1600 seconds. 